\title{
JAS
}

Journal of Analytical Atomic Spectrometry

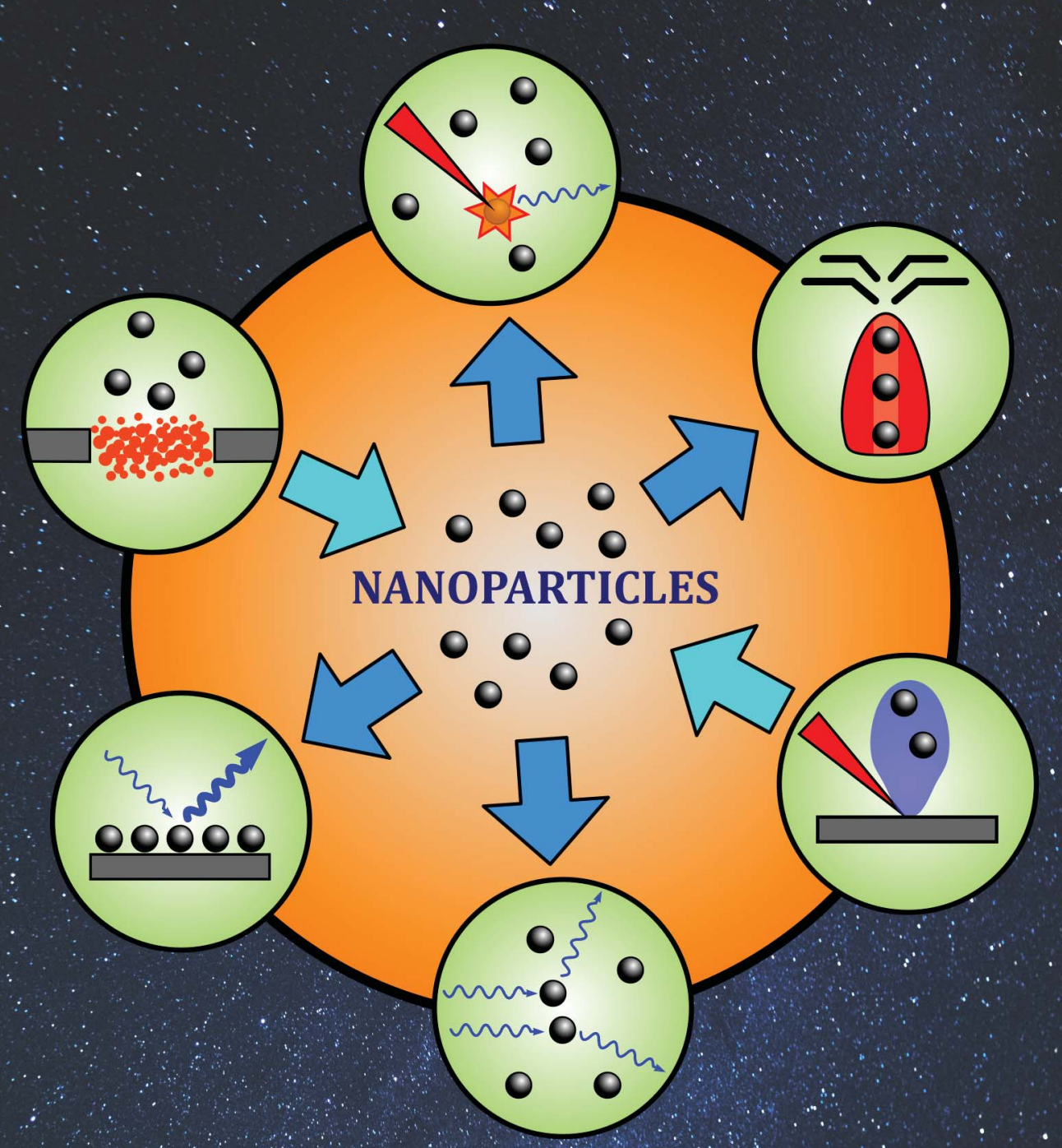


Check for updates

Cite this: J. Anal. At. Spectrom., 2021 36, 1826

Received 30th April 2021 Accepted 14th July 2021

DOI: $10.1039 / \mathrm{d} 1 \mathrm{ja} 00149 \mathrm{c}$ rsc.li/jaas

\section{Nanoparticles in analytical laser and plasma spectroscopy - a review of recent developments in methodology and applications}

\author{
G. Galbács, (D)*ab A. Kéri, ${ }^{\text {ab }}$ A. Kohut, (D) bc M. Veres (D) d and Zs. Geretovszky (DD bc
}

The present review attempts to comprehensively overview the progress in the field of nanoparticle-related analytical laser and plasma spectroscopy research, focusing on the results of the past decade. The discussion involves the brief description of the motivation and principle of operation behind all existing technologies. As a novel approach, the connection between nanoparticles and laser and plasma spectroscopy is discussed in all three major areas: monitoring of nanoparticle synthesis, nanoparticle characterization, as well as plasmonic signal enhancement achieved by using nanoparticles. In each area, a detailed description of methodological developments and modern applications is provided.

\section{Introduction}

Nanoscience has moved forward with giant leaps in the last two decades. The special optical, mechanical, magnetic and

\footnotetext{
${ }^{a}$ Department of Inorganic and Analytical Chemistry, University of Szeged, Dóm sq. 7, 6720 Szeged, Hungary.E-mail: galbx@chem.u-szeged.hu

${ }^{b}$ Department of Materials Science, Interdisciplinary Excellence Centre, University of Szeged, 6720 Szeged, Dugonics sq. 13, Hungary

${ }^{c}$ Department of Optics and Quantum Electronics, University of Szeged, Dóm sq. 9, 6720 Szeged, Hungary

${ }^{d}$ Department of Applied and Nonlinear Optics, Institute for Solid State Physics and Optics, Wigner Research Centre for Physics, 1121 Konkoly-Thege M. way 29-33, Budapest, Hungary
}

energetic properties of nanoparticles (NPs) have been widely recognized and are being exploited in an array of industrial, scientific and medical fields. Today, numerous synthesis technologies are available for the controlled production of engineered NPs, nanostructures and nanocomposites not only at the laboratory, but also at the industrial scale. ${ }^{1-3}$ The influence of nanostructured materials on the efficiency and cost of, or approaches to industrial and scientific processes is so immense that many scientists consider the most recent decades as the first ones in the "nano age", which interweaves with the silicon era.

Analytical science also benefits from the special properties of nanomaterials, as it not only uses these materials, but also

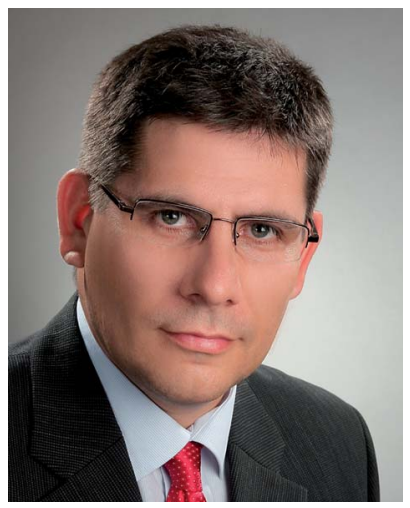

Gábor Galbács is holding MSc diplomas in chemistry and physics, as well as in environmental sciences (University of Szeged, Hungary). He obtained a PhD/CSc degree from the same university in 1998 and a DSc title from the Hungarian Academy of Sciences in 2013, both in the field of analytical chemistry. His research is diverse, but focuses on fundamental studies and instrumentation/method development for laser and plasma analytical spectroscopy, mostly involving LIBS, ICP-MS and ICPAES. He is a full professor and the head of the Department of Inorganic and Analytical Chemistry at the University of Szeged since 2014.

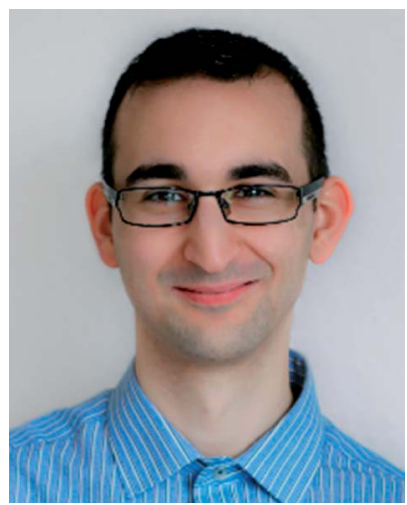

Albert Kéri received his B.Sc. in Environmental Engineering (2014) and his M.Sc. in Chemistry (2016) from the University of Szeged, Hungary. The topic of his PhD thesis - that he defended in 2020 at the University of Szeged - was ICP-MS-based analytical method development for the investigation of multicomponent nanoparticles. He participated in several national and European founded research and $R+D$ projects where he developed ICP-MS and LIBS based analytical methods. Currently he is a postdoctoral researcher at the Inorganic and Analytical Chemistry Department at University of Szeged. 
contributes to or provides inspiration for the development of new ones. Among others, sample preparation and separation techniques, ${ }^{4,5}$ chemical sensing ${ }^{.6-8}$ and spectroscopy ${ }^{9-12}$ are the fields of analytical science that have the most interaction with nanoscience. Instrumental analytical techniques are also crucial to the characterization of $\mathrm{NPs}^{13}$ as well as for the monitoring of nanostructures either during controlled synthesis ${ }^{\mathbf{1 4 , 1 5}}$ or when released in the environment. ${ }^{16}$ This close interaction between analytical science and nanoscience has already produced a number of scientific results, which have been overviewed in several books ${ }^{17-19}$ and review papers. ${ }^{9,20-23}$

Laser and plasma spectroscopic (LPS) techniques are presently dominating the field of analytical spectroscopy. Plasmabased spectroscopy techniques, such as inductively coupled plasma optical emission and mass spectrometry (ICP-OES and ICP-MS), microwave induced optical emission spectroscopy (MIP-OES), glow discharge optical emission and mass spectroscopy (GD-OES and GD-MS), are naturally used mainly for elemental and isotopic analysis, whereas laser sources can serve both atomic and molecular analyses, depending on the laser fluence applied and the analyte reservoir. Examples include

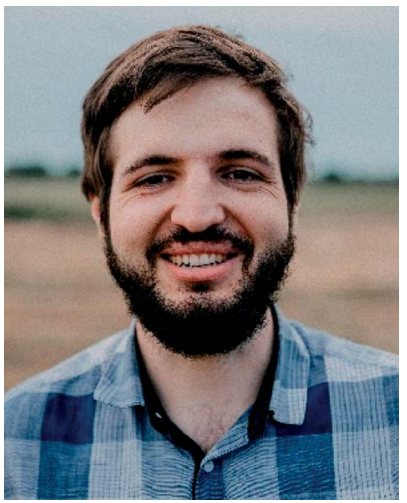

Attila Kohut completed his PhD in physics at the University of Szeged, Hungary in 2018. His PhD research was centered around the fundamental investigation of spark plasmas used for nanoparticle generation. Currently, his research is focusing on the generation of bimetallic nanoparticles in electrical discharges and their applications in different fields.

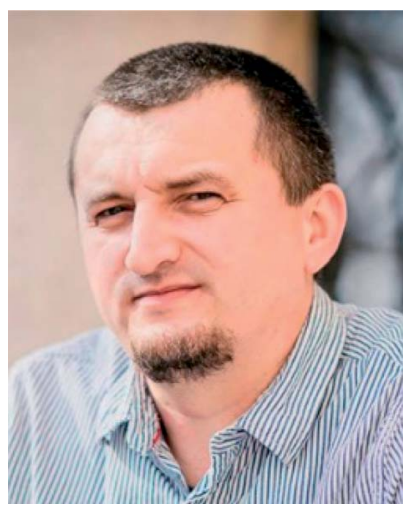

Miklós Veres graduated in physics at the Uzhhorod National University (Uzhhorod, Ukraine) in 2000. He received a PhD degree from the Budapest University of Technology and Economics in 2005 and a DSc title from the Hungarian Academy of Sciences in 2021, both in the field of physics. His research focuses on optical spectroscopy including spontaneous and stimulated Raman scattering, photoluminescence, plasmonic enhancement, spectroscopic imaging and instrumentation development. He is a senior researcher and the head of the Department of Applied and Nonlinear Optics at the Wigner Research Centre for Physics (Budapest, Hungary). laser-induced breakdown spectroscopy (LIBS), laser ablation (LA) sample introduction, Raman spectroscopy, laser-induced fluorescence spectroscopy (LIFS), photoacoustic spectroscopy (PAS), laser enhanced ionization spectroscopy (LEIS), cavity ringdown spectroscopy (CRDS) and more. ${ }^{24}$ More recently, the combination of laser and plasma sources for analytical spectroscopy purposes is also gaining momentum, used either as a performance booster (e.g. ref. 25 and 26), or in tandem (hyphenated) instruments in order to provide more, atomic plus molecular, analytical information about the sample. In the latter bin, often are instruments which involve LA and LIBS, for the reason that these techniques lend versatile sampling and spatial resolution capabilities to other analytical spectroscopies. LIBS-Raman, ${ }^{27}$ LIBS/LA-ICP-MS, ${ }^{28}$ LA-GD-MS ${ }^{29}$ and LIBS$\mathrm{LIF}^{30}$ are examples for such tandem instruments.

Nanomaterials are also more and more involved with lasers and plasmas. For instance, nanoparticle synthesis by electrical discharge plasmas ${ }^{\mathbf{1 , 3 1}}$ or via laser ablation $^{\mathbf{3 2}}$ are recently becoming increasingly common methodologies. Since the most popular engineered NPs are metallic therefore laser and plasma based atomic spectroscopies are most often called for the in situ monitoring of the synthesis process or for the characterization of the produced particles. LIBS ${ }^{33-35}$ and ICP-MS seem to show the best performance and practicality in these applications. In particular, the single particle ICP-MS (spICP-MS) technique has become a very powerful and versatile nanoparticle characterization approach ${ }^{36,37}$ for nanosols. Laser light scattering ${ }^{38}$ and absorption methods (such as $\mathrm{PAS}^{39}$ ), are also well established, important tools of nanoaerosol characterization. Analytical signal enhancement in LPS is also often effectuated by using nanoparticles, mostly based on plasmonic effects. A premier example for this is surface enhanced Raman spectroscopy (SERS), ${ }^{10}$ but LA-ICP-MS ${ }^{40}$ and LIBS $^{12,41}$ applications are also benefiting from similar effects. Last, but not least, the use of NPbased liquid sample preparation methodologies in LPS in

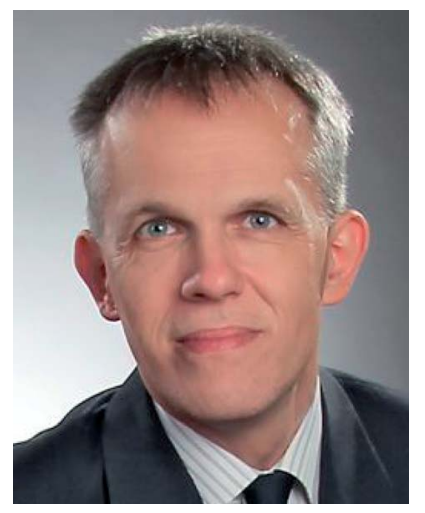

Zsolt Geretovszky holds an MSc in Physics and Chemistry, and a PhD in Physics, both from the University of Szeged (USZ, Hungary). He has been a research fellow at the University College London (UK) and Lund University (Sweden) and currently holds an associate professor position at USZ, where he is the head of the Nano- and Microprocessing Laboratory. Dr Geretovszky has expertise in the generation - especially in the gas phase and laser-based synthesis and characterization of nanoparticles/nanomaterials. He coauthored more than 60 peer-reviewed publications and led several Hungarian and international $R \& D$ projects. Currently he is supervising the work of one postdoctoral and 5 doctoral fellows. 
general, such as separation or preconcentration, are also becoming more frequent in recent literature.

Several reviews overviewed earlier the applications of nanomaterials in analytical science in general, ${ }^{\mathbf{9}, 20-23}$ but so far none focused on the field of laser and plasma spectroscopy, while this field enjoys a steadily increasing involvement of nanomaterials in recent years. The closest scope was provided by the review of Jiang et al. ${ }^{9}$ in 2012 , which covered applications in analytical atomic spectrometry. Therefore, the present review attempts to encompass and comprehensively overview the recent developments in all main areas of laser and plasma spectroscopy which interact with inorganic nanoparticles: namely the monitoring of the synthesis of nanoparticles, the detection and characterization of nanoparticles and the use of nanoparticles for signal enhancement. Please note that the scope does not include single macromolecule analysis. Our review focuses on results that appeared in the literature in about the last decade. Brief overviews of the methodologies of included subfields will be provided, along with references to seminal books and pioneering works, but the focus is on recent applications.

\section{Monitoring of nanoparticle generation}

\subsection{Importance, driving force and overview}

NP synthesis methods range over numerous techniques, including chemical, physical, mechanical, and even biological approaches. ${ }^{\mathbf{4 2 - 4 4}}$ While there are some "golden standards" of NP characterization, such as transmission electron microscopy, certain generation methods have their unique characterization toolbox which fits the conditions of the given production method best. This is especially true when the monitoring of the whole synthesis process is aimed. Laser- and plasma-based spectroscopic techniques proved useful for in situ monitoring of particle formation in a temporally and/or spatially resolved and - potentially - minimally invasive manner. Plasma spectroscopic monitoring is a natural choice when NP generation process includes a plasma stage, such as in the case of laser ${ }^{45}$ or spark $^{46}$ ablation, but it can also be useful when plasma is generated from the already formed particles by means of an energetic excitation source, such as a laser pulse. ${ }^{47}$ The development of the spectroscopic toolbox used for monitoring plasma-based NP generation dates back long before the introduction of the term "nanoparticle". Although in early research the occurrence of microscopic particulates was mostly considered as a side-effect, ${ }^{48}$ the accumulated knowledge on material removal from the target, ${ }^{\mathbf{4 9}}$ on the excitation of different species, ${ }^{50}$ or on the determination of important plasma parameters $^{51}$ provided valuable contribution to the plasma spectroscopic monitoring of current NP generation techniques. The application of lasers in monitoring the NP generation opens up further possibilities via selectively exciting particle populations even at different stages of their formation. Various approaches exist, depending on the laser-particle interaction initiated, such as laser-induced incandescence (LII), ${ }^{52}$ laserinduced breakdown spectroscopy (LIBS), ${ }^{53}$ elastic, $^{54}$ or inelastic ${ }^{55}$ scattering, or absorption, ${ }^{56}$ to mention but a few. In the following sub-chapters, we will summarize some of the most recent laser- and plasma spectroscopy methods applied for the monitoring of NP generation, after a brief description of the plasma diagnostic methodology related to this analytical problem.

\subsection{A concise introduction to plasma diagnostics}

Plasma diagnostics incorporates a broad variety of techniques aiming to give a comprehensive description of plasma properties. When plasmas are utilized as sources for NP synthesis, plasma diagnostic tools allow for the precise measurement of synthesis conditions, hence facilitating the better understanding of particle formation mechanisms as well as providing more control over the generation process. Plasmas are different from other, more common states of matter, first and foremost in that their most important properties are generally cannot be measured directly. Instead, plasma properties are deduced from observations of physical processes and their effects. ${ }^{57}$ This approach greatly relies on the understanding of plasma physics and chemistry involved in said processes. It rather naturally follows from the above that modeling the plasma, or at least those aspects which are subject of investigation, is often an integral part of plasma diagnostics. Since a complete description of the distribution function of the plasma constituents' position and velocity is not feasible in general, plasma diagnostics usually aims at determining the so-called lower order moments of the distribution function, such as the density, mean velocity, pressure, temperature, and heat flux. Numerous techniques exist for obtaining these values, along with multiple possibilities to categorize the different methods.

One way to sort plasma diagnostic techniques is based on the physical process or property of the plasma that is measured, as proposed by Hutchinson in his very comprehensive book on plasma diagnostics. ${ }^{57}$ By following this approach magnetic, particle flux, and refractive index measurements, the measurement of photon emission from free or bound electrons, as well as the measurement of interaction with electromagnetic waves can be distinguished. Since the detailed discussion of these experimental approaches are far beyond the scope of the present review, we will only briefly overview some of the main aspects of the interpretation of the emission from free and bound electrons, i.e., the topic of plasma emission spectroscopy.

Optical plasma emission spectroscopy (OES) is a versatile tool for diagnosing laboratory plasmas, with a well-established theoretical and instrumentational background. ${ }^{58-60}$ Apart from the identification of species present in a plasma, the two main parameters that are predominantly determined by OES are the number concentration of electrons (electron density, $n_{\mathrm{e}}$ ) and the electron temperature $\left(T_{\mathrm{e}}\right){ }^{61}$ In order to support the following sub-chapter, we will briefly mention some widely used methods for calculating $n_{\mathrm{e}}$ and $T_{\mathrm{e}}$, usually employed during the monitoring of plasma-based NP synthesis.

In case of laser- and electric discharge plasmas, electron concentration is mostly calculated from the spectral broadening 
of emission lines, predominantly assuming that Stark broadening is the dominant mechanism. ${ }^{62,63}$ Stark broadening and line shifting results from the Coulomb interactions between the emitting species and the charge carriers present in the plasma. Due to the availability of precise tabulated data and validated theoretical description, the Stark broadening of $\mathrm{H}$ lines especially the $\mathrm{H}_{\beta}$ line - are widely used to determine the electron concentration in plasmas. ${ }^{64-66}$ If $\mathrm{H}$ atoms are not present in the plasma, other elements can also be used to estimate the electron concentration, however, in this case the accuracy is usually lower, deviations typically in the range of $15-50 \%$ can be found. ${ }^{65,67}$ Even though, various theoretical models exist to interpret Stark-broadened spectral lines ${ }^{\mathbf{6 8}}$ in most of the practical cases electron concentration is estimated based on the comparison of the broadened full width at half maximum (FWHM) or line shift with reference data, ${ }^{69}$ tabulated for various transitions. $^{70-72}$ The following two equations are adapted from ref. 69. Eqn (1) describes the electron concentration as a function of the Stark-broadened FWHM for hydrogen and hydrogenlike ions:

$$
n_{\mathrm{e}}=C\left(n_{\mathrm{e}}, T_{\mathrm{e}}\right) \Delta \lambda_{\mathrm{s}}^{3 / 2}
$$

where $\Delta \lambda_{\mathrm{S}}$ is the FWHM of the Stark component, and $C\left(n_{\mathrm{e}}, T_{\mathrm{e}}\right)$ is a coefficient, which is only a weak function of the electron temperature and concentration. Eqn (2) is valid for two- or more-electron atoms:

$$
n_{\mathrm{e}}=n_{\mathrm{e}}^{\mathrm{ref}} \frac{\Delta \lambda_{\text {total }}}{2 w(1+1.75 A(1-0.75 R))}
$$

where $\Delta \lambda_{\text {total }}$ is the total FWHM of the spectral line, $w$ is the electron-impact half-width, $A$ is the ion broadening parameter describing the relative importance of collisions of ions with respect to the broadening, and the $n_{\mathrm{e}}^{\text {ref }}$ is the reference electron concentration, at which $w$ and $A$ are measured or calculated. $w$ and $A$ only slightly depend on the electron temperature and concentration. $R$ describes the ratio of the mean distance between ions and the Debye radius. For eqn (2) to be valid, $A \leq$ 0.5 and $R \leq 0.8$ must hold. For singly charged emitters, the term $0.75 R$ in eqn (2) should be replaced by $1.2 R$.

It should be noted that in some cases the effect of other line broadening mechanisms (such as instrumental, Doppler, resonance, van der Waals broadening) cannot be neglected, therefore the contribution of the Stark effect to the overall line width - often called Stark width - must be determined. To this end, various deconvolution procedures can be used depending on the relative importance of the different mechanisms. ${ }^{73,74}$ In certain cases, deconvolution procedures allow for the simultaneous derivation of electron concentration from the Stark component and the electron temperature from other mechanisms, such as Doppler ${ }^{73}$ or van der Waals ${ }^{75}$ broadening. It should be mentioned that different approaches also exist for determining the electron concentration from the emission spectrum of a plasma, e.g., by using absolute irradiance methods, which usually require the calibration of the spectrometry setup and the modelling of the emission spectrum. ${ }^{76,77}$
The other important plasma parameter, which can be derived by means of plasma OES, is the electron temperature, $T_{\mathrm{e}}$. It should be noted that plasmas are generally described by several different temperature values, corresponding to different plasma constituents (such as electrons, atoms and ions) or processes (such as atomic excitation and molecular vibrations or rotations). In many cases, these temperatures are not equal, hence different approaches are needed to determine a specific temperature value. However, there are certain cases, when some sort of equilibrium can be assumed, thus making temperature determination much easier. This is the case when local thermodynamic equilibrium (LTE) prevails in the plasma. Simply speaking, LTE means that different species present in the plasma have the same local temperature. ${ }^{78}$ To reach LTE, the electron concentration needs to be sufficiently high in the plasma facilitating collisions with electrons to be dominated over radiative processes. In this case, a single "LTE temperature" can be derived, representative to the temperature of the electrons as well. Here we will briefly overview the most popular method used for estimating $T$ from the emission spectrum of a plasma assumed to be in LTE, usually called the Boltzmann plot method. As suggested by the name, it is related to the Boltzmann equation, which describes the population of excited energy levels as a function of temperature. ${ }^{58}$ Since the emission line intensities characteristic to different transitions of an atom or ion are proportional to the number concentration of the species in the corresponding excited states, a relation can be found between the emitted line intensities $\left(I_{i j}\right)$ and the so-called excitation temperature governing the population of the excited states, described by the following equation:

$$
I_{i j}=h \nu_{i j} A_{i j} \frac{1}{Z} g_{i} n \mathrm{e}^{\left(\frac{-E_{i}}{k T}\right)}
$$

where $A_{i j}$ is the transition probability, $Z$ is the partition function, $g_{i}$ and $E_{i}$ are the statistical weight and the energy of the excited level, respectively, $k$ and $h$ are the Boltzmann and Planck's constant, respectively, $T$ is the excitation temperature, $\nu_{i j}$ is the frequency of the transition, and $n$ is the number concentration of the studied species. After rearranging eqn (3), one can obtain

$$
\ln \left(\frac{I_{i j}}{\nu_{i j} g_{i} A_{i j}}\right)=\ln \left(\frac{h n}{Z}\right)-\frac{E_{i}}{k_{\mathrm{B}} T}
$$

It is apparent from eqn (4) that the excitation temperature, which in LTE equals to $T_{\mathrm{e}}$, can be derived from the measured line intensities and the atomic data corresponding to the relevant transitions. A minimum of two emission lines are needed to calculate the temperature by using the Boltzmann equation often called line-pair method, ${ }^{79}$ or two-line method ${ }^{\mathbf{8 0 , 8 1}}$ - but the results are generally more reliable when more spectral lines are involved, preferably with a wide spread over the upper energy level. In this case, the temperature is given by the slope of the graph described by eqn (4). ${ }^{\mathbf{5 1 , 8 2}}$

As it was already noted, Boltzmann plot method relies on the assumption that local thermodynamic equilibrium (LTE) - at 
least partially, for the corresponding transitions - exists. The validity of LTE can be estimated based on the electron concentration from the Griem ${ }^{83}$ or McWhirter ${ }^{84}$ criteria. Even though these criteria are widely used to justify the presence of LTE in atmospheric pressure laser- and arc or spark plasmas, ${ }^{51,69,85}$ there are indications that a more thorough analysis should be made to assess the validity of the assumption of LTE. ${ }^{86}$ When deviation from LTE prevails, the Boltzmann plot method can still be employed to derive the excitation temperature by introducing correction factors. ${ }^{51}$ Nevertheless, the temperature determined from the Boltzmann plot method describes the temperature of the electrons only when the presence of LTE is justified.

Originally, the Boltzmann plot method can be applied to a single species in a given ionization level. However, by exploiting the fact that in LTE the population of different ionization stages are determined by $T_{\mathrm{e}}$ and $n_{\mathrm{e}}$, one can extend the method to successive ionization stages. The method that incorporates this extension is called the Saha-Boltzmann method, named to refer to the fact that the ionization equilibrium is described by the Saha equation (eqn (5)): ${ }^{51,69,87}$

$$
n_{+}=\frac{1}{n_{\mathrm{e}}} 2 \frac{Z_{+}}{Z}\left(\frac{2 \pi m_{\mathrm{e}} k_{\mathrm{B}} T}{h^{2}}\right)^{\frac{3}{2}} \exp \left(-\frac{W_{+}-\Delta W}{k_{\mathrm{B}} T}\right) n
$$

where $n_{\mathrm{e}}$ is the number concentration of electrons, $W_{+}$is the ionization energy of the studied species and $\Delta W$ is the lowering of the ionization energy. ${ }^{88}$ The + subscript refers to ions. Including successive ionization stages widens the energy range in the Saha-Boltzmann plot, as exemplified in Fig. 1, allowing for a more reliable determination of the slope of the linearized equation. However, since the combined Saha-Boltzmann equation is a more complicated function of $T_{\mathrm{e}}$ and $n_{\mathrm{e}}$, this method normally requires an iterative approach to obtain the temperature. ${ }^{65,85}$ When further assumptions can be made about the plasma, such as its stoichiometry, the Saha-Boltzmann

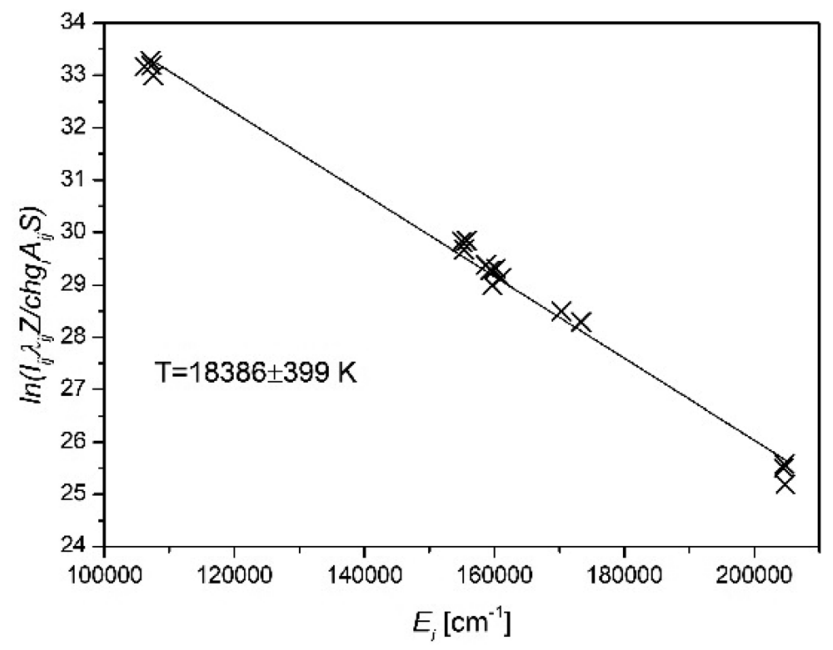

Fig. 1 Saha-Boltzmann plot constructed from the emission spectrum of a Cu/Ar spark discharge plasma measured ca. $1 \mu$ s after the onset of the gas breakdown (based on the authors' own data). method can be extended to different elements as well. Due to the involvement of multiple elements at potentially different ionization stages, the so-called multi-element Saha-Boltzmann method allows for an even more precise determination of the LTE temperature of the plasma. ${ }^{89}$

We have already mentioned the importance of LTE in Boltzmann plot methods, but there are several additional considerations which should also be kept in mind. Here we only briefly list some of these considerations, for further details please refer to the cited literature. First, it is important to note that the accuracy of constants involved in the equations highly affect the accuracy of the calculated values. The most critical factor here is the transition probability, which in many cases is only known with poor accuracy. ${ }^{65}$ It should also be noted that the above methods are applicable for optically thin plasmas. Therefore, when self-absorption is dominantly present in the emission spectra, additional techniques or corrections must be applied. ${ }^{69,90-92}$ It should also be kept in mind that OES techniques provide spatially integrated information over the line of observation. Therefore, spatial deconvolution techniques might need to be applied. For instance, a commonly used technique for optically thin plasmas with cylindrical symmetry is the Abel inversion. By employing Abel inversion, the radial intensity distribution can be reconstructed from the lateral intensity distribution. ${ }^{69}$

The above-mentioned methods rely on the line emission of the plasma for temperature determination, but additional OESbased plasma diagnostic techniques also exist. The continuum radiation emitted by plasmas - highly characteristic to the early stages of laser ablation plasmas $^{69}$ - can be exploited for temperature calculation via the so-called line-to-continuum intensity ratio method. As suggested by its name, this method employs both the intensity of the spectral lines and the continuous component of the emission spectrum to derive the electron temperature under LTE conditions. ${ }^{93}$ Apart from the importance of sufficiently well-known parameters and constants, which cannot be stressed enough at all OES-based methods, the applicability of this approach is also limited to those stages of plasma evolution when line spectrum coexists with continuum radiation, both having sufficient signal-tonoise ratio. ${ }^{69}$

It should also be mentioned that LTE conditions are often not met in many plasmas, however, OES- (and laser spectroscopy) based methods are still proved useful in the description of these plasmas. An important parameter of non-LTE plasmas is the rotational temperature of molecules present in the plasma, which is often assumed to be equal to the - translational - gas temperature. There are various techniques for obtaining the rotational temperature from emission (and absorption) spectra, which were critically reviewed recently by Bruggeman et al. ${ }^{94} \mathrm{We}$ only mention that when an equilibrium rotational population is assumed, essentially the Boltzmann plot method can be applied to the determination of the rotational temperature, with the difference that rotational spectral lines must be used for constructing the plot. Simulating the rotational spectrum and fitting to its measured counterpart is also a commonly used method for deriving the rotational temperature. This approach 
has the advantage that depending on the complexity of the model used for generating the synthetic spectrum, more thorough description of the analyzed plasma can be given than in case of equilibrium-based methods.

\subsection{Methodology and applications}

Laser- and plasma spectroscopic methods are predominantly used in the in situ monitoring of NP generation in particle synthesis techniques where the initiation of a plasma is involved, such as electrical discharges (mostly arcs and sparks), laser ablation, and flames. Therefore, in the following subchapters we will focus on the monitoring methodology applied in these synthesis methods, while some further synthesis routes where in situ laser- or plasma-based particle monitoring were utilized will be mentioned at the end of the chapter.

2.3.1 Monitoring of flame synthesis. Flame synthesis is a well-established gas-phase method for the production of inorganic NPs, predominantly metal oxides and carbon black. ${ }^{95}$ Considering the amount of the produced particles, flame synthesis is the most widely used NP generation technique by far, mainly used for the synthesis of $\mathrm{SiO}_{2}, \mathrm{TiO}_{2}$, and $\mathrm{Al}_{2} \mathrm{O}_{3}$ on industrial scale. ${ }^{96}$ Flame aerosol reactors are based on the controlled burning of a gaseous or liquid precursor, which yields NPs in the range of several hundreds of grams per hour. ${ }^{96}$ Due to its exceptional capability for producing NPs on a large scale, the technique was subjected to extensive academic and industrial interest. ${ }^{97}$ This is justified by the highly complex nature of the processes taking place in a flame, necessitating the combination of knowledge in molecular chemistry, reaction kinetics, thermodynamics, and materials science. ${ }^{97}$ From a point of view of the generated NPs, it is especially important to assess the temperature-time history of a particle, one of the most significant parameters determining the properties of the final product, which can be estimated by in situ temperature and velocity measurements. ${ }^{98}$

Since optical diagnostic methods are capable of providing this information without disturbing the synthesis process, a broad range of techniques have been developed or applied to the specific conditions of flame synthesis. Fig. 2 schematically shows the particle generation in a flame reactor and exemplifies several optical and non-optical diagnostic tools for the in situ and ex situ investigation of different aspects of the particle formation process. Optical diagnostic methods applicable for monitoring flame synthesis were thoroughly reviewed very recently by Dreier and Schulz, ${ }^{100}$ Schulz et al. ${ }^{101}$ and Rahinov et $a .^{102}$ therefore we only recall here some of their main considerations supplemented by related literature.

Laser-based methods applicable for the in situ monitoring of flame NP synthesis can be divided into two main groups, the first includes the methods appropriate for monitoring the gasphase particle formation process (such as the temperature and intermediate species concentration), while the second group consists of the techniques capable of monitoring the properties of the generated particles. ${ }^{\mathbf{1 0 0}}$ A commonly used method both for temperature and concentration measurements is based on the absorption of a laser beam. Information on the average temperature or concentration of intermediate species (such as small molecules corresponding to precursor reactions) can be gained by measuring the absorption spectrum by using ring-dye lasers or tunable diode lasers, employing Fouriertransform infrared spectroscopy (FTIR), intra-cavity laser absorption spectroscopy (ICLAS), or cavity-ring-down absorption spectroscopy (CRDS). ${ }^{\mathbf{1 0 0}}$ Even though absorption-based techniques provide line of sight information, by employing fan-shaped laser illumination and multiple detectors, temperature and concentration distributions can be reconstructed using a 1D tomographic algorithm. ${ }^{103}$ Another method enabling the online monitoring of the flame temperature and the concentration of the intermediate species is laser-induced fluorescence (LIF). LIF methods are usually distinguished by the species the measurements are based on, such as $\mathrm{NO}$ or $\mathrm{OH}$ for temperature, and various other species (e.g., $\mathrm{Fe}, \mathrm{CH}_{2} \mathrm{O}, \mathrm{CH}$, $\mathrm{OH}, \mathrm{SiO}, \mathrm{FeO}$, In, AlO, TiO) for intermediate concentration determination. ${ }^{\mathbf{1 0 0}}$ Recently, the good applicability of SiO-LIF in quantitative temperature ${ }^{\mathbf{1 0 4}}$ and concentration ${ }^{\mathbf{1 0 5}}$ imaging as well as the investigation of the combustion chemistry of the flame synthesis of $\mathrm{SiCl}_{4}$ nanoparticles ${ }^{\mathbf{1 0 6}}$ have been demonstrated.

In addition to the above techniques, the temperature can also be determined based on both elastic and inelastic scattering of laser light. Raman- and coherent anti-Stokes Raman scattering (CARS) as well as Rayleigh scattering have been applied to measure the temperature in flame reactors, ${ }^{\mathbf{1 0 0}}$ however, since these techniques are more suitable for particlefree environments, usually they are of limited use in strongly particle-laden flows. ${ }^{\mathbf{1 0 1}}$ Apart from temperature measurements, light scattering-based techniques can also be used to monitor the properties of the as formed particles in situ. For this purpose, another laser-based technique is the so-called laserinduced incandescence (LII), which is based on the measurement and analysis of the incandescence signal of the particles heated up by a laser beam, providing information on volume fraction and primary particle $\operatorname{size}^{\mathbf{1 0 0}}$ (see Section 3.3.2. for details). Since the proper interpretation of the LII signal is not straightforward, attempts are made to standardize calibration and signal processing protocols. ${ }^{107,108}$ When the laser fluence is high enough to vaporize the NPs, the emitted atomic spectrum can also be used to gain information on the particles. This is the basis of laser-induced breakdown spectroscopy (LIBS), a technique well-known in analytical spectrochemistry ${ }^{109}$ (see Section 3.3.3. for details). In situ aerosol LIBS is relatively challenging to execute and interpret the results, but it has been shown to be able to provide valuable information on the complex processes taking place in turbulent flame reactors. ${ }^{\mathbf{1 1 0}}$

2.3.2. Monitoring of laser ablation synthesis. NP generation by lasers is based on the ablation of a solid target by an energetic laser beam in a gaseous or liquid environment. Particle formation will take place through several steps from the material ejected off the surface, the exact process of which is depending on the laser parameters (wavelength, pulse width, fluence), the target material, and the surrounding medium (gas, liquid, or vacuum). ${ }^{45}$ The particles can be collected in the form 


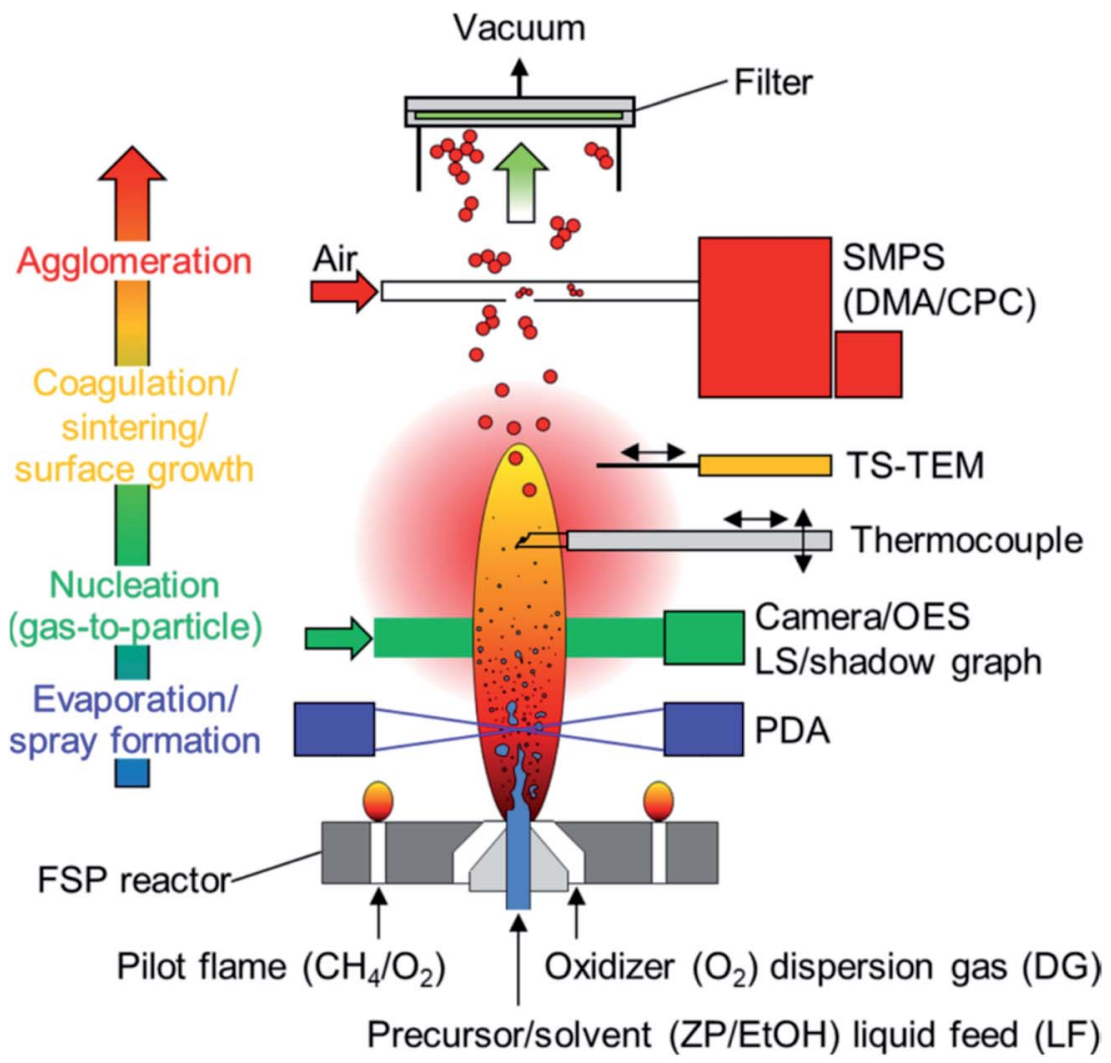

Fig. 2 Schematic figure of an experimental flame spray pyrolysis (FSP) reactor setup and process diagnostics for analyzing the consecutive stages of metal oxide NP evolution. Nonintrusive laser and camera diagnostics including phase-Doppler anemometry (PDA), laser sheet (LS) and shadow graphic (SG) for liquid spray analysis (blue), as well as optical emission spectroscopy (OES) and camera imaging for determination of the metal oxide nanoparticle nucleation zone (green). Thermophoretic sampling with subsequent transmission electron microscope analysis (TS-TEM, orange) and scanning mobility particle sizer (SMPS, red) are applied for local description of primary and agglomerate nanoparticle evolution, while filter collected particles are considered for ex situ analysis (BET and XRD). Figure and caption are taken from ref. 99.

of a nanopowder, colloid, or they can be deposited onto a substrate to form a thin layer. The latter is the basis of the well-established technique of pulsed laser deposition, or PLD. ${ }^{\mathbf{1 1 1}}$ Laser-based NP generation is associated with the formation of a plasma plume over the ablated surface, consisting of neutral and excited species of the target material. The emission or the absorption of this plasma can be investigated by various spectroscopic and plasma diagnostic methods. In fact, laser ablation NP generation mostly accompanied by the creation of a plasma plume, which is the very basis of LIBS. ${ }^{112,113}$ Due to this common root, instrumentation and methodology developed for LIBS can naturally be adapted to the monitoring of laser ablation NP generation. Similarly to that of the monitoring of electrical discharges (see in the next sub-chapter), this is predominantly based on the acquisition of the emission of the plasma and the calculation of the main plasma parameters, such as the electron concentration and temperature.

The most basic approach for the monitoring of the laserinduced plasma (LIP) is the collection of its temporally and spatially integrated radiation by means of an optical fiber during the ablation process. Thereafter, e.g., the Boltzmann plot method (see in Chapter 2.2.) can be applied to calculate the excitation temperature of the plasma, ${ }^{\mathbf{1 1 5 , 1 1 6}}$ or the Stark broadening to derive the electron concentration. ${ }^{\mathbf{1 1 7}}$ Naturally, much more reliable data can be obtained when the highly transient LIP is monitored with appropriate temporal resolution. Evolution of electron concentration as derived from Stark broadening, ${ }^{118-120}$ temperature evolution calculated from the Boltzmann plot $^{\mathbf{1 2 0}}$ and Saha-Boltzmann plot, ${ }^{118,121}$ or relative line intensity ${ }^{\mathbf{1 1 9}}$ methods are routinely applied. It should be noted that even in case of temporally resolved measurements, care should be taken when temperature calculation methods relying on the existence of LTE are applied. This is especially true in case of low-pressure laser ablation experiments where electron concentration decreases rapidly, hence LTE can only be assumed in the earliest stages of the laser plasma. ${ }^{\mathbf{1 2 2}}$ Another circumstance, which necessitates high temporal resolution is the limited temporal detectability of the emission signal, as in case of pulsed laser ablation under liquid (PLAL), where the signal is detectable for only a short period of time (a few tens of nanoseconds to a few microseconds). Nevertheless, by employing a high temporal resolution ICCD camera, the evolution of the plasma emission can be monitored ${ }^{\mathbf{1 2 3}}$ and the plasma temperature can be deduced in this short interval e.g., by fitting the continuum component of the emission spectrum by a Planck-like distribution, ${ }^{\mathbf{1 1 4}}$ or by employing the Boltzmann 
plot method to the spectral lines. ${ }^{\mathbf{1 2 4}}$ A typical experimental setup for OES-based monitoring of NP generation during PLAL is shown in Fig. 3. The temperature can also be deduced by comparing the measured and simulated vibrational and rotational spectra of the plasma, supplemented by electron concentration determination from the Stark broadening of spectral lines. ${ }^{\mathbf{1 2 5}}$

Apart from the calculation of plasma parameters (such as temperature or electron concentration), the intensity variation of specific spectral lines also carries important information on the particle generation process. Such information for instance is the transfer of species dissolved in a liquid into the plasma created during PLAL. By following the intensity variation of two ionic lines the spectral features can be correlated to the composition of the ablated sample and thus the transfer process can be investigated. ${ }^{\mathbf{1 2 6}}$ Similarly, by comparing the emission intensity of an atom to that of the ion or oxide of this atom, the dynamics of the ionization or oxidization processes can be monitored. ${ }^{\mathbf{1 1 8 , 1 2 5}}$ Direct information on the variation of atomic or ionic spectral lines is also useful when spatially and temporally resolved OES is employed to monitor one or more process parameters of the laser ablation process, such as the ablation medium (e.g. gas or liquid) ${ }^{\mathbf{1 1 9}}$ ablation wavelength ${ }^{\mathbf{1 2 1}}$ or the ambient pressure. ${ }^{127-130}$ Having spatial, temporal, and spectral resolution at the same time allows for carrying out time-of-flight measurements of specific species generated during laser ablation. The expansion dynamics of selected plasma constituents can be derived from the emission spectra and used for instance in correlation with the excitation processes, ${ }^{130}$ or to detect the onset of the deposition process and the growth of a NP film during PLD. ${ }^{\mathbf{1 2 1}, 127,131,132}$

It should be noted that temporally and spatially resolved OES is predominantly carried out by acquiring a relatively broad spectrum of a localized spatial region with a ns-gated camera, thus obtaining information on several species at the same time. However, this indicates that an extended area can only be covered by "scanning", i.e., repeating the measurements in different locations. In contrast, when the spatio-temporal investigation of only one or a few specific spectral lines is aimed, high spatial and temporal resolution can be achieved at once by imaging the plasma after using a wavelength-dispersive element. ${ }^{\mathbf{1 3 3}}$ Additional information can be gained on the particle formation process by employing a second laser pulse to excite the species ablated by the main pulse. The second pulse can be either collinear ${ }^{\mathbf{1 1 4}}$ or orthogonal ${ }^{\mathbf{1 2 4}}$ to the main pulse, and the emission of the excited species can be used to calculate the excitation temperature via the Boltzmann plot method, or the electron concentration from Stark broadening. ${ }^{114}$ A remarkable feature of this double-pulse approach is the correlation of the emission generated by the second pulse with the changes

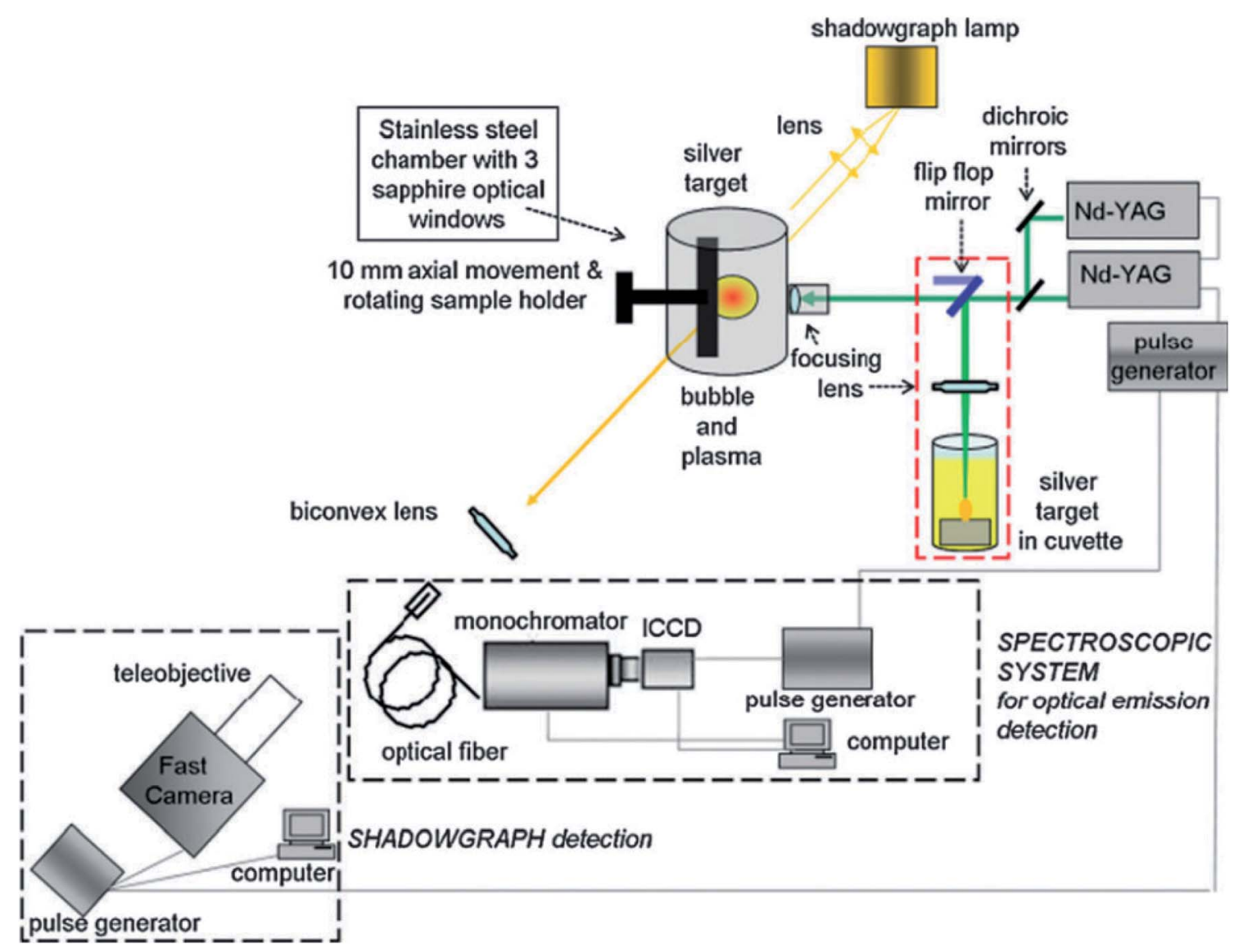

Fig. 3 Schematic of the experimental setup for the OES-based monitoring of NP generation by pulsed laser ablation in gas and liquid. ${ }^{114}$ 
associated with the first pulse, be it the dynamics of bubble formation under liquid, ${ }^{\mathbf{1 1 4}}$ or the composition of the generated nanoparticles. ${ }^{\mathbf{1 2 4}}$

In the above cited studies, optical emission of the lasergenerated plasma was acquired and analyzed during NP generation to monitor the particle synthesis process. However, different approaches also exist, which use plasma- and/or laser spectroscopic techniques for the in situ characterization of the laser ablation-generated particles and therefore monitor the generation process. Dynamic light scattering (DLS) is a widely used technique to investigate NPs dispersed in a liquid, ${ }^{\mathbf{3 8 , 1 3 4}}$ see Section 3.3.1. for details. Wei and Saitow have demonstrated that DLS can be used in connection with a pulsed laser ablation NP generation system for the in situ monitoring of particle synthesis in a supercritical fluid. ${ }^{\mathbf{1 3 5}}$ As opposed to the seconds to hours temporal domain covered by the DLS measurements of Wei and Saitow, temporal resolution in the picosecond range can also be achieved via femtosecond transition absorption spectroscopy. By employing sub-picosecond pump and probe laser beams, the evolution of the transient absorption spectrum of gold NPs exposed to pulsed laser irradiation could be followed. ${ }^{136}$ In situ probing a femtosecond laser plasma was carried out by Oujja et al. They have employed a second, nanosecond laser pulse perpendicular to the material ablating laser to generate its third harmonics and obtained its spatiotemporal distribution from nanoseconds to hundreds of microseconds after ablation. It was concluded that the harmonic generation is affected by the formation of middle-sized metal clusters that had a huge effect on the harmonic generation yield. Thus, this nonlinear optical approach is capable of investigating the cluster formation during fs-laser ablation-based NP generation. ${ }^{137}$ All the previous methods exploited the investigation of the optical response of the laser plasma or the generated NPs, induced by laser excitation. However, as demonstrated by Valverde-Alva et al., the photoacoustic signal generated during the laser ablation of a target in ethanol can also be used to monitor the NP generation process. ${ }^{138}$ Their results exemplify well that the acoustic signal generated by the ablating laser pulses is also useful for the in situ monitoring of laser ablationbased NP synthesis.

2.3.3. Monitoring of electrical discharge synthesis. Electrical discharges generally involve the (partial) ionization of a gas or a liquid by an electric field, facilitating the flow of electrical current through this ionized medium. ${ }^{139}$ Such a phenomenon can occur in various forms, resulting in various types of different discharges depending on the experimental conditions. Although NPs can be generated by virtually any type of discharge, from a spectroscopic monitoring point of view it is worth distinguishing between discharges forming thermal and non-thermal plasmas. Thermal or hot plasmas can be described by some form of equilibrium (most often LTE), meaning that species are characterized by the same temperature, at least in a given spatial point. ${ }^{86}$ On the contrary, non-thermal or cold plasmas are characterized by higher electron temperature than that of the heavy species, therefore LTE conditions are not fulfilled. ${ }^{140}$ Most prominent examples of the latter are dielectric barrier discharges, ${ }^{\mathbf{1 4 1}}$ glow discharge,${ }^{\mathbf{1 4 2}}$ or several types of spark discharges. ${ }^{143}$ Thermal plasmas include atmospheric-pressure (transferred) arcs, ${ }^{\mathbf{1 4 4}}$ and atmospheric-pressure, microsecondlong, oscillatory sparks. ${ }^{85}$ In the following, we will discuss the spectroscopic monitoring results of the latter two types of discharges in more detail for the main reason that in the past few decades the experimental techniques relying on arc and spark plasmas have attracted considerable scientific and industrial interest and became prominent candidates for satisfying the need for the mass production of high purity, engineered NPs. ${ }^{46,144-146}$

Electric sparks are not self-sustaining discharges, meaning that after the initiation of the breakdown of the medium between the electrodes, energy must be continuously supplied externally to maintain sparking. ${ }^{\mathbf{1 4 7}}$ The way of the energy input, the design of the electrodes or the surrounding medium may vary, thus greatly influencing the discharge characteristics, including the existence or non-existence of LTE in the discharge plasma. One form of spark discharges associated with the formation of a thermal plasma is utilized in the so-called spark discharge nanoparticle generators (SDGs). ${ }^{\mathbf{1 4 8}}$ SDGs are based on the spark ablation of a pair of electrodes placed in a gas-tight chamber under atmospheric pressure. The sparking is maintained by periodically discharging a capacitor (typically having a capacitance in the range of a few or a few tens of $\mathrm{nF}$ ) connected to the electrodes. The sparking is associated with an energetic discharge plasma allowing for high currents (several hundreds of amperes) to flow between the electrodes typically for a few microseconds, resulting in the atomization of the electrode material. This material is quickly cooled down due to expansion and the presence of a gas stream continuously flowing through the interelectrode gap. This gas flow also carries away the ablated material and facilitates the formation of NPs. ${ }^{\mathbf{1 4 9 , 1 5 0}}$ The generated NPs are dispersed in the carrier gas thus forming an aerosol. Aerosol science has well established methodologies to analyze the produced particles both in situ ${ }^{151-153}$ and ex situ. ${ }^{154}$ However, an inherent limitation of these techniques is that they only provide information about the "final product" of the process - i.e., the NPs - and therefore unable to monitor the generation process itself, especially the initial plasma stage.

Optical diagnostics of thermal spark plasmas has a long history with well-established methodology and scientific results, mostly driven by the research interest in the field of analytical spectrochemistry. Spark plasmas include transient, temporally and spatially varying phenomena the reliable investigation of which necessitates the use of temporally - and preferably spatially - resolved techniques. ${ }^{155}$ Due to the analytical perspective, the early studies in the field mostly focused on the direct analysis of the emitted light in order to understand the sampling and excitation mechanisms taking place in the spark. ${ }^{156}$ Those studies mostly included the determination of the concentration of the ions and electrons by utilizing the Stark effect, ${ }^{64}$ or laser scattering, ${ }^{157}$ or interferometry. ${ }^{158}$ Calculation of the plasma temperature by means of the Boltzmann ${ }^{159}$ or the Saha-Boltzmann plot method ${ }^{51}$ has also been carried out. A comprehensive overview of the methods applicable for gas discharges can be found in the book by Boumans, ${ }^{50}$ while the 
fundamental mechanisms obtained during the analysis of the spark emission is reviewed by Scheeline. ${ }^{49}$

These results serve as an important basis for the optical monitoring of electrical discharge-based NP generation; however, they cannot be directly applied for modern SDGs. The reason for this is, in contrast to the highly regulated and wellcontrolled analytical sparks, SDGs are mostly operated in "free running mode" when sparking occurs as soon as the breakdown voltage of the interelectrode gap is reached. ${ }^{160}$ The lack of regulating circuitry results in a technically simple and easy-to-construct design and a higher particle yield, but also hinders the use of external instrumentation with precise active triggering and sometimes poses stability issues. Other technical constraints, originating from e.g., the generator chamber or the components of the gas line also introduce physical limitations which narrows the range of applicable methods. All the above considerations rather naturally lead to the use of OES as a useful spectroscopic technique applicable under the circumstances of NP generation. In its most basic form, OES can be applied simply to analyze the emission originating from the spark gap without spatial or temporal resolution. Hontañón et al. used an optical fiber to collect the light emitted by the spark plasma during NP generation and analyzed its spectrum by a simple tabletop spectrometer. The acquired spectra reflected the spatially and temporally averaged spectrum of several concomitant sparks but still provided useful information about the conditions of the generation process. They have collected the spectra of a continuous glow and arc discharge used for NP generation as well and demonstrated that even with such an experimentally simple OES approach, the three discharge regimes are clearly discernible. ${ }^{\mathbf{1 6 1}}$ This has great potential in the optical monitoring of electrical discharge-based NP generators.

A much deeper insight can be gained by acquiring timeresolved spectra of the spark plasma, an experimental setup for which is shown in Fig. 4. In a similar arrangement, Kohut et al. employed a fiber-coupled echelle spectrograph equipped with an intensified CCD to investigate the temporal evolution of the spectrum emitted by a spark plasma during NP generation, with the concomitant fast imaging of the sparking, with a temporal resolution of $50 \mathrm{~ns} .^{85}$ Due to the already mentioned unregulated nature of the sparking, the spectral acquisition was synchronized to the plasma formation by using the sharp drop of the capacitor voltage as a trigger signal. This introduced a $c a$. 100 ns time delay, which inherently means that the very first stages of a spark discharge - i.e., the so-called pre-breakdown and breakdown stages ${ }^{\mathbf{1 6 2}}$ - cannot be investigated by this approach. These temporal periods, however, are considered to have minor contribution to NP generation, compared to the following - arc and afterglow - stages. ${ }^{15}$ The temporally resolved, though spatially integrated, spectra also allowed for reconstructing the intensity evolution of the atomic lines of the electrode material, which correlated well with the evolution of the discharge stages. The time-resolved data allows for the identification of temporal windows in which LTE holds, therefore the Boltzmann plot method (see Chapter 2.2.) can be applied to obtain the temperature-variation of the spark plasma, thus deriving its cooling rate. ${ }^{15}$ The fundamental information, which can be gained from the time-resolved emission spectrum of the spark discharge can be enriched by increasing the spatial resolution of the spectral acquisition. In case of an SDG operating with argon carrier gas and copper electrodes, the applicability of the Saha-Boltzmann method was demonstrated for calculating the evolution of the LTE temperature of the plasma in its first $c a .2 .5 \mu$ s after the onset of the breakdown. ${ }^{85}$ In the same period, the electron concentration in different spatial positions could also be deduced from the Stark broadening of selected lines of argon ions. The sufficient temporal and spatial resolution also allowed for using the

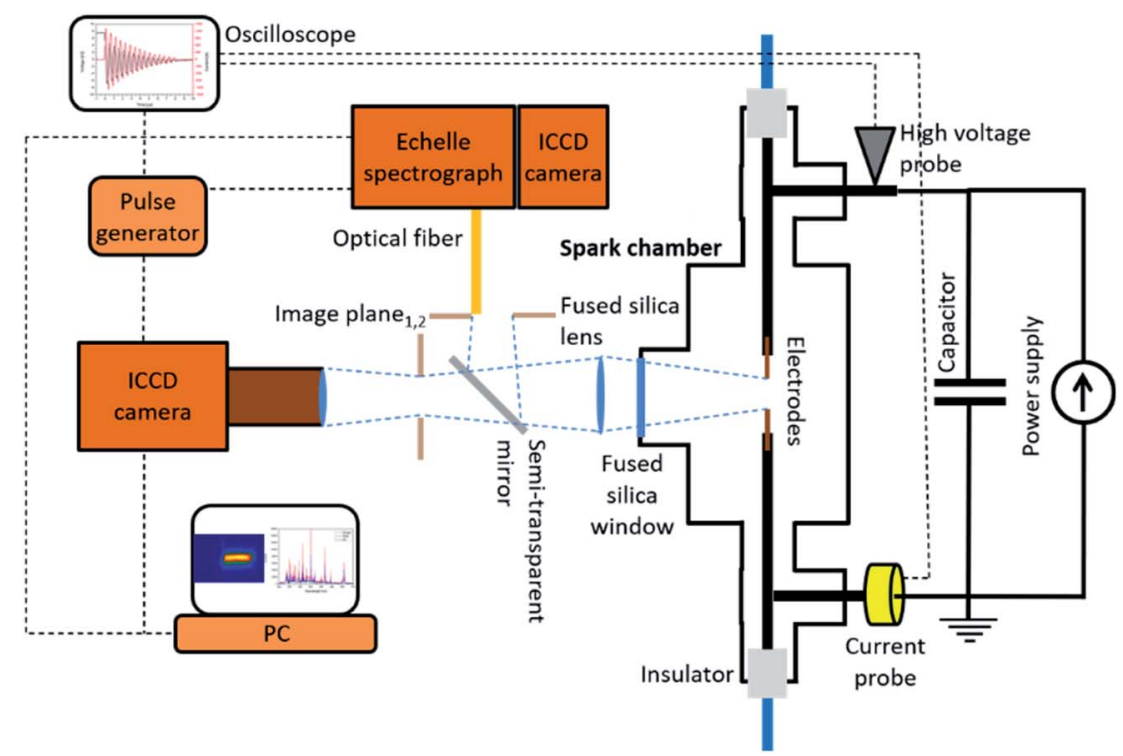

Fig. 4 Schematic view of the experimental setup used for the in situ OES-based monitoring and imaging of a spark discharge nanoparticle generator. 
collected spectral data as an input for a semi-empirical plasma model, which was able to describe the cooling stage of the spark plasma (afterglow). Moreover, due to the spatially resolved spectral acquisition, the spatial distribution of the concentration could also be deduced. ${ }^{85}$

Although SDGs are most commonly operated in argon or nitrogen, pulsed discharges are also widely applied for producing NPs under a liquid environment. ${ }^{163-165}$ The working liquids usually consist of hydrogen, which allows for the application of the well-established methodology for deriving the electron concentration via the Stark-broadening of $\mathrm{H}$ lines. ${ }^{166,167}$

As it was mentioned earlier, another type of gas discharge used for nanoparticle generation - both in gas and liquid - is the thermal arc plasma. ${ }^{78}$ Arc discharge NP generators (ADGs) have the advantage over SDGs that due to the continuous regime and hence the lack of a capacitor and high voltage charging power supply, an even simpler and cheaper technical design can be realized. ${ }^{\mathbf{1 6 8}}$ ADGs are characterized by high particle yield and very good scalability, however the particle sizes are typically considerably larger than that of sparkproduced NPs and its applicability for producing high purity and multielement particles is also limited. ${ }^{168}$ From the point of view of spectroscopic measurements, arc plasmas have an even more established historical background, on which the monitoring of ADGs can be based. ${ }^{50}$ Generally, this includes all those plasma diagnostic techniques discussed in Chapter 2.2. and utilized for SDGs. However, due to the different plasma characteristics there are additional methods which can be employed. One important distinction from sparks is that depending on the exact experimental conditions - the emission spectrum of the arc consists of a continuous background originating from the blackbody radiation of the electrodes. ${ }^{161}$ This can be simply exploited for temperature estimation in addition to other - already described - techniques, such as the Boltzmann plot and line-intensity-ratio methods, or for electron concentration measurements based on the Stark broadening. ${ }^{169,170}$ In ADGs operating under different experimental conditions molecular bands of the ambient nitrogen gas was observed in the spectrum, the intensity variation of which along the arc plasma was correlated to the properties of the produced nanostructures. $^{169}$ Similarly, in case of underwater or submerged arcs, the features of the temporally resolved emission spectra were used to assess the peculiarities of the electrode erosion process and the degree of dissociation of water in the vicinity of the anode. ${ }^{171}$

In the previous paragraphs, the importance of spatial and temporal resolution in OES-based monitoring of arc and spark plasmas was pointed out and appropriate methods have been reviewed. These approaches mostly used well-localized signal collection with variable spatial positioning combined with a spectrograph. However, spatial resolution can be achieved in a single step by employing an imaging camera with appropriate optical filters. Bachmann et al. have proposed a method for obtaining the temperature distribution of a free-burning arc plasma, based on the simultaneous imaging of the plasma at two carefully chosen wavelengths. ${ }^{172}$ The experimentally acquired data was processed by using an LTE plasma model to derive the plasma temperature. Abel inversion of the data was used to reconstruct the radial distribution of the arc plasma column. A detailed assessment of the potential sources of errors and uncertainties was also provided.

In addition to the detection and analysis of the light emitted by the discharge plasma, the in situ monitoring of the particle generation has been also approached via laser-based methods. Santra et al. have shown theoretically that NPs can be detected via Rayleigh scattering spectroscopy during arc synthesis, along with the continuous monitoring of the evolution of the particle population. ${ }^{173}$ Rayleigh scattering spectroscopy is based on the probing of the electronic polarizability of particles interacting with a laser beam. Since Rayleigh scattering is elastic, this technique has the advantage over for instance Raman spectroscopy that the signal levels are orders of magnitudes higher. ${ }^{173}$ Another technique, which pushes down the detection size limit to the atomic scale and applicable to the in situ monitoring of arc synthesis is the coherent Rayleigh-Brillouin scattering (CRBS). CRBS is originally a laserbased gas detection technique, which was adapted to NPs and experimentally validated by Gerakis et al. ${ }^{174}$ CRBS relies on the creation of an electrostrictive grating and the probing of the velocity distribution in a medium via a four-wave mixing process. According to Gerakis et al. this technique can be effectively used to detect NPs in situ in volumetric particle generation methods, such as arc synthesis. ${ }^{174}$ The formation of arc-produced carbon nanostructures was also monitored in situ by using laser-induced incandescence measurements and simulations by Yatom et al. ${ }^{175}$ The viability of their approach was demonstrated via the recognition of two spatially separated group of particles with distinctly different sizes, having different role in the arc-based NP formation process. ${ }^{175}$ At higher laser fluences the particles can be effectively vaporized, which facilitates their in situ investigation by means of aerosol mass spectrometry. The successful application of this technique for investigating spark-generated NPs has been demonstrated by Nilsson et al. ${ }^{153}$

As it was mentioned at the beginning of this chapter, spectroscopy-based methodology briefly overviewed here are mostly applicable to thermal plasmas, where most of the LTEbased plasma diagnostic calculations can be carried out. However, OES-based techniques are involved in the investigation of cold plasmas as well. Particle generation by cold plasmas (produced by e.g. filamentary discharges or dielectric barrier discharges $)^{\mathbf{1 7 6}}$ can also be monitored by spectroscopic means. Due to the relatively low gas temperature molecular spectral bands are usually observable. The gas temperature can be determined by simulating the molecular spectrum and finding the best fit of its measured counterpart. ${ }^{177-179}$ Processing the experimentally acquired data is usually carried out by dedicated purpose-made software and scripts but commercial software is also available for fitting the spectra of several transitions observed in plasmas and for calculating the corresponding temperatures. ${ }^{180}$

\subsection{Other generation techniques}

In the previous subchapters we have briefly reviewed the laserand plasma diagnostic techniques used for monitoring NP 
generation in plasma-based methods of high relevance from the point of view of applications. There are other additional particle generation methods as well, where the applicability of laser- and plasma diagnostic monitoring has been demonstrated. An example for such techniques is the continuous laser vaporization, which - unlike pulsed laser ablation - employs a continuous wave, usually $\mathrm{CO}_{2}$ laser to vaporize a precursor, predominantly for producing carbon nanotubes. ${ }^{181}$ Similarly to the monitoring of flames, CARS, LIF, and LII have been employed for the in situ monitoring of temperature, intermediate concentration, as well as the assessment of the particle formation process. ${ }^{182}$ In a similar, laser-based particle generation scheme, the applicability of in situ LIBS has also been demonstrated to monitor the properties of the generated particles. Picard et al. employed aerodynamic focusing of the NPs under vacuum in order to eliminate the contribution of the background gas from the LIBS spectrum. ${ }^{183}$ Low-temperature, low-pressure reactive plasmas are also successfully used to generate nanostructures, ${ }^{\mathbf{1 8 4}, \mathbf{1 8 5}}$ the formation of which can be monitored by laser spectroscopic approaches. Hundt et al. have employed quantum cascade laser absorption spectroscopy for the real time monitoring of the variation of the acetylene concentration in an $\mathrm{Ar} / \mathrm{C}_{2} \mathrm{H}_{2}$ plasma during nanostructure synthesis in a radio frequency (RF) plasma. ${ }^{\mathbf{1 8 6}}$ Leparoux and coworkers have investigated the synthesis process of graphene nano-flakes in $\mathrm{Ar} / \mathrm{H}_{2} / \mathrm{CH}_{4} \mathrm{RF}$ inductively coupled plasmas by using in situ OES. ${ }^{187,188}$ The acquired emission spectra allowed for the determination of the gas temperature from the $\mathrm{C}_{2}$ Swan band, which could be correlated to the particle generation conditions. A recent review on the monitoring of non-thermal plasmas used for NP synthesis has been published by Mangolini. ${ }^{189}$

Particle generation techniques overviewed in the previous subchapters in context of real-time laser- and plasma spectroscopic process monitoring employed various types of precursors (solid, liquid, gas), in different media (liquid, solid, vacuum), but both included plasmas to initiate particle formation. However, laser spectroscopy has been also used to monitor wet chemical NP synthesis. Haber and coworkers used in situ second harmonic generation ( $\mathrm{SHG}$ ) and extinction spectroscopy to monitor the growth dynamics of seed-mediated gold and gold-silver core-shell NPs, ${ }^{\mathbf{1 9 0 , 1 9 1}}$ which demonstrates the even wider possibilities of laser-based NP monitoring approaches.

\section{Nanoparticle detection and characterization}

\subsection{Importance, driving force and overview}

As it is known, the size and structure of nanomaterials are mainly responsible for their novel properties (electric, chemical, magnetic, optical, etc.), so naturally the characterization of nanomaterials has become the subject of intense research in the past couple of decades. Unfortunately, the fact that their size and structure critically determine their characteristics also makes the assessment of their physico-chemical properties (or structure-function relations) challenging, because their synthesis is prone to reproducibility problems and generally produces polydisperse particles, frequently with a broad distribution and defects. In addition to this, their characterization needs a comprehensive analytical approach, which also dictates the knowledge of limitations of the different techniques. As nowadays nanoparticles are being synthesized and used at an industrial scale, also including medical applications, ${ }^{192,193}$ it has led to their inevitable appearance in the environment, which in turn generated an urge for the development of sensitive and credible techniques for their detection.

Since there is no universal characterization method that could simultaneously determine all important NP parameters, typically the use of complementary techniques is required. NP systems can be investigated in powder or suspension form but in certain cases the dissolution of particles is needed. Here follows a brief overview of the NP characterization methods. Further information about these techniques and their application in various media can be found in specific reviews and books..$^{13,14,194-199}$

Electron microscopy techniques represent one of the most frequently applied group of NP characterization methods. Scanning electron microscopy (SEM) utilizes a well-focused electron beam that scans the surface of the sample and reflected electrons are used for imaging, while in transmission electron microscopy (TEM) a thin (typically less than $200 \mathrm{~nm}$ ) sample interacts with the electron beam and the transmitted electrons are collected. Their popularity is derived from the fact that through "visualizing" NPs, a wide set of important characteristics can be determined, such as particle morphology, size distribution, degree of aggregation and - using a highresolution apparatus - even crystal structure.

Scanning probe microscopes move a very sharp tip across the solid sample surface. In scanning tunneling microscopy (STM), DC voltage is applied between the conducting sample and the tip and the tunneling current, which is formed if the distance of the two object is very small (nm scale), is monitored. Atomic force microscopy (AFM) is based on electrostatic interactions between the atoms of the tip and the sample. Both STM and AFM allow for an atomic scale resolution imaging, hereby providing information on the morphology of nanomaterials. They can even be utilized to create structures in the sub-nano range by manipulating atoms or molecules on the sample surface. ${ }^{200,201}$

Due to different electromagnetic radiation-matter interactions, a great number of NP parameters can be studied by X-ray techniques. Complementing electron microscopy with energydispersive X-ray (fluorescence) spectroscopy (EDS or EDX) the elemental composition and its distribution in single NPs can be determined. Small angle X-ray scattering (SAXS) provides information about the size and size distribution, shape, and specific surface area of NPs. X-ray diffraction (XRD) probes ordered structures (crystal planes); hence the crystal structure, lattice parameters and crystallite size of the investigated nanomaterial can be determined. X-ray photoelectron spectroscopy (XPS) utilizes the photoelectric effect to perform surface analysis with an information depth of $2-10 \mathrm{~nm}$. XPS is capable of the investigation of electronic structure, elemental composition, 
and oxidation states of elements in the surface of nanomaterials and its contaminants. Using appropriate data evaluation, particle size, single and multiple coatings, shell thicknesses and surface functionalization of NPs can be studied. ${ }^{202}$

UV-visible absorption spectroscopy is a simple and low-cost but not very selective characterization method which utilizes that the optical properties of NPs significantly depend on the size, shape, number concentration and degree of aggregation of the particles. NPs with intensive localized surface plasmon resonance in the $\mathrm{UV}$-visible range (e.g. $\mathrm{Au}, \mathrm{Ag}, \mathrm{Cu}$ ) are particularly often studied by this method. ${ }^{203}$ Infrared spectroscopy (IR) can also be utilized for certain nanomaterial characterization aims. The position of infrared absorption bands bears information on nanoparticle-biomolecule conjugation, conformational states, and secondary structures of the bound proteins. ${ }^{\mathbf{2 0 4}}$

Atomic spectroscopic analytical techniques are widely used for nanoparticle detection and characterization. This is due to that their high selectivity permits the analysis even in complex sample matrices and their high sensitivity allows the investigation of small nanoparticles present at very small amounts. Inductively coupled plasma mass spectrometry (ICP-MS) ${ }^{\mathbf{2 0 5}}$ and graphite furnace atomic absorption (GFAAS) techniques, ${ }^{206}$ should be mentioned as particular examples, traditionally exploited for the determination of the composition and mass concentration of nanodispersions.

In this chapter, we will focus on the NP characterization and detection techniques which are based on laser and plasma spectroscopy. Due to the vastness of the field, this overview obviously cannot be complete, but we will attempt to present the actual trends, relevant methodologies, and application examples. In the following sub-chapters, we organized the discussion according to the spectroscopic detection principle (e.g. absorption, emission, mass spectroscopy, etc.).

\subsection{Techniques based on the interaction of nanoparticles with laser beams and plasmas}

Laser-particle and plasma-particle interactions play a central role in techniques used for the detection or characterization of nanoparticles. These analytical techniques are specifically based on the light absorption, scattering or fluorescence of particles or on the detection of emission of photons or chemical species/fragments released (emitted) by the particles upon their interaction with a laser beam or plasma.

The nature of the interaction involved in the measurement as well as the analytical benefit/information obtained vary among the techniques involved. A range of laser or plasma based analytical measurement techniques associated with NP detection and analysis are in use today; Table 1 provides an overview of the most important ones.

The interaction of nanostructures with light is a complex topic (essentially it encompasses the whole revolutionary field of nanophotonics) and therefore the discussion of its details is way beyond the scope of this chapter. Here, we would only like to point out to some of the specifics of laser-particle interactions relevant in laser and plasma spectroscopy. One aspect clearly involves the excitation of the surface plasmons (oscillations of free electrons) of metal or alloy NPs. Plasmonic resonances can be exploited in laser spectroscopy by generating signal enhancement (amounting to several orders of magnitude), as is discussed in the examples of SERS or NE-LIBS spectroscopies, in Chapters 4.3.2. and 4.3.3. Laser light, especially Q-switched giant-pulses, carry huge electrical field strengths (e.g. $10^{9}$ to $10^{11} \mathrm{~V} \mathrm{~m}^{-1}$ ) which when interacting with the very high curvature NPs, can easily produce field emission of electrons. These can serve as seed electrons that lower the laser ablation and laser-induced breakdown thresholds, thereby facilitating analytical techniques such as LA-ICP-MS or LIBS. ${ }^{207}$ Light scattering is a well-known optical phenomenon during which the incident light perturbs electrons in the structure to form oscillating dipole moments that re-emit the light in different directions. The intensity and direction of this scattered light depend both on the incident wavelength and specific properties of the structure. Light scattering is exploited for the size and concentration characterization of NPs in techniques such as DLS and NTA. Furthermore, it has been shown recently both theoretically and experimentally that if the light interacts with subwavelength structures (NPs) then superscattering can occur. This effect, in the present context, may result in a further decrease of the size detection limit of certain NPs. ${ }^{208}$ Absorption of laser light by aerosol particles is exploited in techniques such as laser desorption and ionization mass spectroscopy (e.g. MALDI, or LDI-MS), laser-induced fluorescence (LIF) and photoacoustic spectroscopy (PAS) for the characterization of the chemical composition and size distribution. Individual particles can also be trapped optically and then interrogated by laser beams. ${ }^{209}$

In the context of detection and characterization, the interaction of nanostructures with plasmas is typically a breakdown process. The analysis of nano- and microparticles (including suspensions and aerosols) using plasma spectroscopy basically falls within the interest of atomic spectroscopy, where the plasma serves as the high temperature atomization/ionization source. Major examples include ICP and LIB plasmas. Detailed experimental and numerical simulation studies are available in the recent literature which investigate the propagation and decomposition of particles introduced into analytical plasma sources, as well as the influence of instrumental conditions on the analytical signal formation (e.g. ref. 210-212). Of particular importance here is single particle ICP-MS, discussed in detail in Chapter 3.3.6.1., which is rapidly becoming a popular and versatile nanoparticle characterization technique.

\subsection{Methodology and applications}

3.3.1. Laser scattering. Static and dynamic light scattering techniques have been routinely used for micro- and nanoparticle detection and sizing for a long time in suspensions. Technologies based on this detection approach have matured; optical particle counters (OPCs), granulometers (often referred to as multi-angle light scattering, MALS), angular scattering instruments (also known as laser diffraction or LD instruments), dynamic light scattering (DLS, also known as photon 
Table 1 An overview of laser-particle and plasma-particle interactions utilized in NP characterization spectroscopies

\begin{tabular}{|c|c|c|c|}
\hline Name of technique & Type of interaction & Principle of measurement & Analytical benefit/information \\
\hline $\begin{array}{l}\text { Laser-induced breakdown } \\
\text { spectroscopy (LIBS) }\end{array}$ & $\begin{array}{l}\text { Laser-particle and } \\
\text { plasma-particle }\end{array}$ & $\begin{array}{l}\text { Detection of characteristic light } \\
\text { emission generated during the } \\
\text { breakdown (or the vicinity) of } \\
\text { particles in the LIB plasma }\end{array}$ & $\begin{array}{l}\text { Detection of the presence of NPs } \\
\text { and estimation of the composition/ } \\
\text { size/concentration of NPs dispersed } \\
\text { in gaseous samples } \\
\text { Signal enhancement for liquid or } \\
\text { solid samples (NELIBS) }\end{array}$ \\
\hline $\begin{array}{l}\text { Inductively coupled plasma mass } \\
\text { spectrometry (ICP-MS) }\end{array}$ & Plasma-particle & $\begin{array}{l}\text { Statistic evaluation of detected MS } \\
\text { signal peaks caused by the } \\
\text { breakdown of particles in the ICP } \\
\text { plasma }\end{array}$ & $\begin{array}{l}\text { Detection and detailed } \\
\text { characterization }(e . g . \text { concentration, } \\
\text { composition, size, structure, shape, } \\
\text { density, porosity, etc.) of NPs } \\
\text { dispersed in gaseous or liquid } \\
\text { samples }\end{array}$ \\
\hline $\begin{array}{l}\text { Laser ablation inductively coupled } \\
\text { plasma mass spectrometry (LA-ICP- } \\
\text { MS) }\end{array}$ & $\begin{array}{l}\text { Laser-particle and } \\
\text { plasma-particle } \\
\text { (at different locations) }\end{array}$ & $\begin{array}{l}\text { Laser ablation of a solid sample in } \\
\text { the vicinity of NPs, followed by the } \\
\text { ICP-MS analysis of the ablated } \\
\text { matter }\end{array}$ & $\begin{array}{l}\text { Imaging of the spatial distribution } \\
\text { of nanoparticles within solid } \\
\text { samples } \\
\text { LA-ICP-MS signal enhancement }\end{array}$ \\
\hline $\begin{array}{l}\text { Laser desorption-ionization mass } \\
\text { spectrometry (LDI-MS) }\end{array}$ & Laser-particle & $\begin{array}{l}\text { MS detection of species/fragments } \\
\text { released (evaporated/desorbed) }\end{array}$ & $\begin{array}{l}\text { Analysis of the composition of } \\
\text { particles }\end{array}$ \\
\hline
\end{tabular}

Laser-induced fluorescence (LIF) Laser-particle

Photoacoustic spectroscopy (PAS) Laser-particle

Nanoparticle tracking analysis

(NTA)

Dynamic light scattering (DLS)

Laser-induced incandescence (LII)

Laser-particle

Laser-particle
Laser-particle from NPs upon their excitation by intense laser light

Detection of characteristic light emission generated during the laser excitation of particles (fluorescence) Detection of characteristic light absorption of particles

Image-based detection of light scattered by particles (can be combined with detection based on LIF)

Image-based detection of light scattered by particles

Laser heating of aerosol particles and detection of the subsequent thermal emission produced
Determination of the distribution, characteristic size or composition of NPs dispersed in a gas (aerosol) Determination of the type or composition of NPs dispersed in gaseous or liquid samples Determination of the characteristic size and concentration of particles dispersed in a liquid

Determination of the characteristic size and concentration of particles dispersed in a liquid

Determination of the characteristic particle size and volume fraction of particles in the observed spatial region correlation spectroscopy, or PCS) and particle tracking analyzers (PTA) are all available commercially. About two dozen ISO and ASTM standards exist that describe the protocols and methodologies of these measurements. Progress in the field in recent years mainly involved the technical aspects of instrumentations (e.g. wavelength of the employed laser shifted to shorter wavelengths to make detection of even smaller NPs possible; semiconductor lasers have replaced $\mathrm{He}-\mathrm{Ne}$ lasers; charge-coupled devices (CCDs) and complementary metaloxide-semiconductor (CMOS) photosensor arrays are more and more commonly used, instruments are more automated and compute faster, etc.), but new experimental and data evaluation approaches also allow the determination of a wider range of physico-chemical characteristics of particles (e.g. zeta potential, ${ }^{\mathbf{2 1 3}}$ diffusion coefficient, ${ }^{\mathbf{1 3 4}}$ viscosity, ${ }^{\mathbf{1 3 4 , 2 1 4}}$ molecular weight for biopolymer particles, ${ }^{215}$ etc.) suspended in liquid media. Due to the popularity and maturity of these technologies, there is a vast amount of literature available, thus only a brief, non-complete account of recent advancements of the field will be provided below. Further information on these topics can be found in selected reviews and books. ${ }^{38,134,213,216-219}$

Instruments falling in this category are based either on the measurement of scattering intensities at various spatial locations (OPC, LD, MALS) and/or as a function of time (DLS), or follow particle movement (PTA). The theory of light scattering by spherical dielectric particles with isotropic optical properties have been worked out a long ago (Mie) and later expanded to other symmetrical shaped particles. At the same time, no exact mathematical solution exists for the description of scattering intensity distributions for arbitrarily shaped and/or optically anisotropic particles, thus in these cases, approximations based on matrix and numerical methods are used (e.g. ref. 220 and 221). For this reason, data evaluation in laser scattering particle characterization techniques require an a priori assumption about the shape of the particle and cannot work with irregular and mixed-shaped particles. In addition, in a liquid medium, these techniques generally require a suitable refractive index contrast between the medium and the particle, a strong 
dilution, as well as a reasonably monodisperse sample, thus prior separation by e.g. size exclusion chromatography (SEC) or field-flow fractionation (FFF), ultracentrifugation, etc., is often needed. Complex samples with strong background fluorescence and scattering from medium-sized molecules can cause difficulties. The smallest particle sizes detectable are typically in the 10-40 nm range, but this value strongly depends on the sample. ${ }^{134,213}$

Today, DLS and NTA are the two most popular particle characterization scattering techniques in suspensions, but NTA has distinct advantages over DLS in the case of NPs. Since DLS is an intensity weighed methodology which measures the fluctuations in scattered light intensity and correlates it to the particle hydrodynamic diameter via the correlation function and the Stokes-Einstein equation, therefore size distribution curves are heavily weighted towards larger particle sizes. As opposed to this, NTA uses a camera to individually identify and track the motion of each particle in the suspension. This gives a betterbalanced size distribution curve; only 50\% difference in particle diameter is needed for successful size resolution, whereas a $c a$. threefold difference is required by DLS - this makes NTA superior for the analysis of polydisperse samples. In addition, NTA is inherently capable of determining the particle concentration..$^{38,134}$

3.3.2. Laser-induced incandescence. Laser-induced incandescence (LII) is a well-established emission-based laser spectroscopy technique, which involves the heating of aerosol particles by laser radiation and then measuring the resulting emission signal produced by the relaxation. The magnitude of the LII signal correlates with the volume fraction of particles in the detection region, and the signal decay rate is mainly influenced by the specific surface of the particles, which depends on the particle size. Pulsed and continuous wave laser light is equally used in this technique. The LII literature has been overviewed by Schulz et $a .^{52}$ and Michelsen et al. ${ }^{222}$ in detail. Numerous application examples also exist for NP analysis.

LII is particularly suitable for the detection of aerosol NPs if the particle has a large extinction, which is true for carbonaceous particles (soot). Incipient soot particles are close to spherical and are small (1-10 $\mathrm{nm})$, mature soot is composed of primary particles of $10-50 \mathrm{~nm}$ in diameter with a fine structure. These primary particles are then covalently bound into nonspherical aggregates of tens to hundreds of nanometers in size. Soots are also very suitable LII measurement subjects, as they have a very high (in excess of $4000 \mathrm{~K}$ ) sublimation temperature and an appreciable imaginary part of the refractive index. Therefore, in environmental and industrial applications, LII has been used extensively for the qualitative measurements of temporal and spatial distributions of soot, but quantitative measurements of volume fractions and primary particle size have also been attempted. ${ }^{52,222}$

Apart from soot, engineered NPs have also been measured by LII due to the increasing interest in the synthesis of nanoparticles in the gas phase. In situ measurement of particle sizes is of particular interest because it offers the possibility of process monitoring/control. In this context, successful analysis of carbonaceous and non-carbonaceous engineered NPs have equally been reported. The list includes e.g. carbon nanotubes, ${ }^{223,224}$ metal-oxide (e.g. $\mathrm{TiO}_{2}, \mathrm{Mn}$ - and Fe-oxides) $)^{225-227}$ and metal/metalloid (e.g. Ag, Cu, Ti, Mo, W, Ni, Fe, Si) $)^{228-230}$ NPs. However, the transfer of the accumulated knowledge in the LII analysis of carbonaceous particles to various inorganic NPs are not without challenges. For example, plasmonic resonances in aggregates of noble metal NPs can experience a highly nonuniform heating. There is also a large uncertainty associated with values of the thermal accommodation coefficient, a central parameter in LII data evaluation, for most combinations of noncarbonaceous particle material and ambient gas. It was recently suggested that molecular dynamics calculations ${ }^{231}$ might be used to tackle this problem.

Laser-induced incandescence is commonly linked to the measurement of nanoaerosols, but investigations that focus on the measurement of suspensions also occur in the literature. This interest is somewhat limited though, since there are commercial laser scattering-based instrumentations available for this purpose (e.g. DLS, NTA). As an example, the study of Sommer et $a l^{232}$ can be mentioned here, in which the authors investigated the pulsed-LII-signal decay times of carbon-black nanodispersions and found a linear correlation with the primary-particle size determined by electron microscopy. There are also reports about black carbon NP measurements in rain and snow samples (e.g. ref. 234).

3.3.3. Laser-induced breakdown spectroscopy. Laserinduced breakdown spectroscopy (LIBS, also known as laserinduced plasma spectroscopy) is an atomic emission spectroscopy technique, which is based on using high irradiance laser pulse(s) to break down, ablate and thermally excite the material of the sample in the focal spot. The resulting high temperature, highly transient microplasma produces intense, characteristic emission. At early times, the emitting species are electrons and ions, but later, as the plasma cools, atoms and - in the last stage - molecules (formed by the recombination of atoms) take over. In order to obtain a spectrum with good signal-to-background (and signal-to-noise) ratio analytical lines, it is necessary to use gated spectroscopic detection. With the optimization of the measurement conditions, ppb-level detection limits and highly characteristic, information-rich emission spectra can be obtained. In the past two decades, LIBS is enjoying a great interest from the analytical plasma spectroscopy community due to its versatility, robustness, selectivity, and sensitivity, not to mention that compact, stand-off, field-capable LIBS instrumentation are also available. ${ }^{109,235,236}$

Utilizing the localized (spatially resolved) analysis capability of LIBS, its application to nanoaerosol (ultrafine particulate matter) or NP detection and characterization has been successfully demonstrated in solid, gas and liquid phases over the years. For example, sub-micron or nanoaerosol particles, both on-line (free-stream, e.g. ref. 33) and off-line (following collection on filters, e.g. ref. 237) aerosol analysis approaches were tested in the literature, since LIBS was first employed for aerosol analysis in the 1980s. ${ }^{238}$ Among others, important contributions to the field were made by Hahn and coworkers $^{239,240}$ as well as by Noll and co-workers, ${ }^{241,242}$ e.g. with respect to sampling statistics, triggering, assessing the upper 
size limitation on the complete ablation of NPs, etc. Aerosol analysis by LIBS in general has been recently reviewed in ref. 240 and 243.

Indicatively, three noteworthy directions of recent LIBS efforts in the field of nanomaterial analysis should be mentioned here: (i) selective analysis of aerosol NPs by low intensity pulses, (ii) characterization of optically trapped single NPs or nanodroplets, and (iii) mapping the distribution of NPs in biological matrices. The following sections outline the progress in these fields.

3.3.3.1. Selective analysis of aerosol nanoparticles. When studying $\mathrm{TiO}_{2}$ nanoaerosols using LIBS, Zhang et al. observed low-intensity atomic emission spectra from $\mathrm{Ti}$ atoms without any macroscopically visible spark although the used laser pulse energy was significantly lower (2-35 $\mathrm{mJ}$ ) than typical values necessary for LIBS aerosol analysis. ${ }^{244}$ At the same time, no spectral lines from the ambient gas (nitrogen) could be observed, indicating that there was no gas-phase breakdown. After a detailed investigation, the authors concluded that under such conditions, a nanoplasma is created which only breaks down the NPs. It was also observed that the atomic emission signal grows with the increase of the size of nanoparticles, reaching a maximum at around $6 \mathrm{~nm}$ (for $\mathrm{TiO}_{2}$ and $532 \mathrm{~nm}$ laser wavelength). The methodology was named low-intensity phaseselective LIBS (PS-LIBS). Two significant advantages of the technique were articulated by the authors: (i) the very short (ns range) lifetime of this nanoplasma allows for the use of similarly short gate-delay times, (ii) the emission spectrum is free from gas lines; therefore, it can be used for the detection of NPs or for the selective monitoring of NP synthesis processes. These advantages were experimentally exploited in follow-up metaloxide NP aerosol studies by the same group ${ }^{245-247}$ and even a theory for the mechanism of absorption-ablation-excitation laser-nanoparticle cluster interactions was developed. ${ }^{248}$ It was established that the threshold irradiance that triggers the formation of nanoplasma is about six times higher for a $1064 \mathrm{~nm}$ excitation wavelength than for $532 \mathrm{~nm}$, resulting in an emission intensity that is nearly 100 times lower in the former case. Based on this observation and the ablation model proposed by the authors involving two-photon excitation of electrons in the conduction band of the metal-oxide particles, it was suggested that an excitation wavelength tuned to above the bandgap of the material of interest should be used. ${ }^{245,248}$ Tse et al. also proposed and successfully demonstrated ${ }^{245,249}$ that the resonance signal enhancement methodology (RELIBS, originally introduced by Cheung et al. ${ }^{250,251}$ ), which involves the use of an additional delayed excitation laser pulse tuned to one of the electronic transitions of a major constituent of the sample (here: particle), can be exploited to produce a more than two orders of magnitude increase of the inherently weak PS-LIBS signals.

3.3.3.2. Characterization of optically trapped nanomaterials. Aerodynamic focusing ${ }^{252}$ and optical trapping ${ }^{253}$ of fine and ultrafine aerosol particles, followed by an investigation of the characteristics of individual NPs is an upcoming analytical approach in the last decade, but LIBS has been applied to trapped NPs and nanodroplets only most recently. The Laserna group in Malaga, Spain, pioneered this approach ${ }^{34,35,254,255}$ and demonstrated that LIBS possesses the attogram-level detection power necessary for such an analytical task.

The Spanish researchers employ their proprietary sample introduction methodology, named optical catapulting (OC), which is based on the ejection (mobilization, suspension) of particles deposited on a glass slide into the surrounding gas, initiated by a laser pulse delivered to the backside of the slide, to put the particulate material under inspection in aerosol form. A cw laser beam at $532 \mathrm{~nm}$ is then used to isolate and manipulate individual NPs from the aerosol (optical trap, OT), which are subsequently analyzed by using a $1064 \mathrm{~nm}$ nanosecond laser pulse for LIBS (Fig. 5). Once catapulted, the dynamics of particle trapping depends both on laser beam characteristics (power and intensity gradient) and on particle properties (size, mass, and shape). The utilized long working distance (low numerical aperture) microscope objective to focus the low power (140-250 $\mathrm{mW}$ ) cw trapping beam allows for a very stable holding of the NPs at atmospheric pressure over a large length (e.g. 5 $\mathrm{mm}){ }^{34,254,255}$ In the latest study of the authors, ${ }^{35}$ an enhanced sampling strategy, based on using skimmer-like cones, was introduced to double the sampling throughput of OC-OT-LIBS technology for single NPs.

Detailed high-resolution imaging with an ICCD camera provided visual feedback on the particle manipulation. Trapping efficiency was defined as the number of catapulting events resulting in a particle entering the optical trap and remaining occluded for a period long enough to proceed with LIBS characterization. Trapping was considered stable once no confined particle-aerosol collisions could be observed in the camera image. 76-93\% trapping efficiencies, half of which were single particle events, were achieved in the studies. Interestingly, an inverse relationship between the emission intensity and particle mass was found, which allows for LIBS measurements with unprecedented sensitivity. With the OC-OT-LIBS, the Laserna group successfully recorded good $\mathrm{S} / \mathrm{N}$ LIBS spectra of individual $100 \mathrm{~nm} \mathrm{Al} \mathrm{O}_{3},{ }^{254} 25 \mathrm{~nm} \mathrm{Cu},{ }^{34} 400 \mathrm{~nm}$ graphite ${ }^{255}$ and $90 \mathrm{~nm}$ copper-oxide ${ }^{35}$ NPs. The sample introduction and analysis only took 3-6 minutes.

Most recently, Niu et al. proposed an optical trapping approach based on the use of a hollow cw laser beam, and successfully demonstrated that OT-LIBS can be applied for the quantitative determination of metal concentrations in individual, $2 \mu \mathrm{m}$ black carbon aerosol particles. ${ }^{256}$ In other studies, nL volume droplets and 1-3 micrometer aerosol particles were also successfully trapped and individually analyzed by LIBS. Järvinen et $a .^{257}$ used an electrodynamic, whereas MenesesNava et al. ${ }^{258}$ an acoustic levitator for the trapping of particles. Since all the above three technologies can spatially constrain aerosol particles to a very small, $\mu \mathrm{m}$-wide range, they are expected to be applicable to ultrafine (nano) particles as well.

3.3.3.3. Mapping of the distribution of NPs in biological samples. LIBS was recently also successfully used to assess the accessibility of NPs to and distribution of nanoparticles in plants and animal tissues. Although there are other techniques capable of providing spatially better resolved chemical maps (e.g. SEM or TEM), but the advantage of LIBS is that it can also 


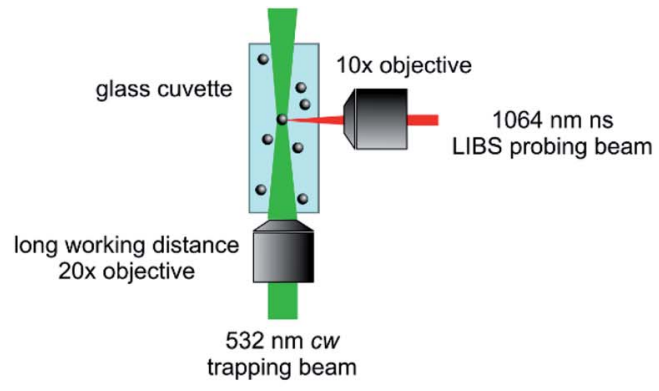

SIDE VIEW

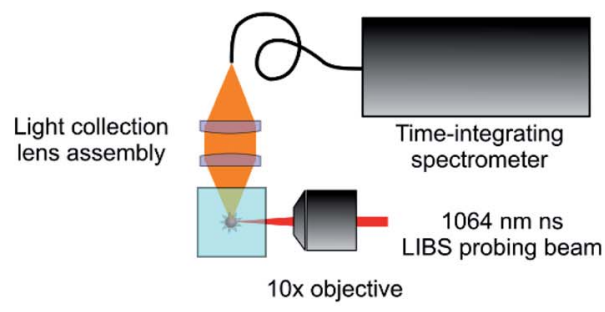

TOP VIEW

Fig. 5 Scheme of an experimental arrangement for LIBS analysis of optical trapped single nanoparticles.

be applied to large samples and it requires no labeling. The compatibility of the LIBS setup with standard optical microscopes also provides a potential to obtain multiple images of the same biological tissue with different types of response including elemental, molecular, or cellular. It is noteworthy that - in contrast to general LIBS solid sample analysis - biological tissue samples need to be prepared for LIBS analysis. Although there is no standardized procedure for this, but it generally involves embedding in epoxy. For soft tissues, a prior dehydration is also needed. Of course, there are variations in the approaches used depending on the tissue type - in the case of nanoparticle detection, thin sectioning (into $10-40 \mu \mathrm{m}$ slices) was also found to be necessary. Further details, e.g. sample preparation, instrumental requirements, data evaluation, on LIBS imaging of various species in biological samples can be found in related recent reviews. ${ }^{236,259-261}$

The research group of Motto-Ros is the pioneer and driving force behind LIBS elemental imaging and NP distribution research in human and animal soft tissues. Most of their investigations serve with information for preclinical medical studies and kinetic biological studies. In 2012, the research group proposed a methodology for the elemental imaging of metal-based NPs in frozen murine biological tissues. ${ }^{262,263}$ The distribution of Gd NPs was imaged in two dimensions at the scale of an entire organ (i.e., kidney or liver). Kinetics studies, in which NPs were injected into animals, were also supported by LIBS NP detection, by screening the organs of elimination at different times after administration of metallic NPs. In 2014, the spatial resolution of the instrumentation was improved to $35 \mu \mathrm{m}$, which enabled the observation that Gd-based NPs were predominantly localized in the renal cortex at early time points before being progressively accumulated in the medulla; the effective renal clearance of Gd NPs was observed one week after the injection. ${ }^{263-266}$ Motto-Ros et al. also used LIBS to investigate the tumor-penetration of NPs. From this point of view, the behaviour of Gd/Si-based ${ }^{267}$ and Au-based ${ }^{268}$ NPs were investigated on paraffin-embedded specimens made from monkey and mice pancreatic and glioblastoma tumor-bearing models. LIBS analysis clearly indicated the presence of metal NPs both in the center and at the periphery of tumors, which confirmed previous results obtained with magnetic resonance imaging (MRI). The lateral resolution achieved in the above studies was strongly impacted by the sample preparation and sample type: 40-100 $\mu \mathrm{m}$ resolution was achieved for cryo-sections, ${ }^{263}$ whereas a resolution of $10 \mu \mathrm{m}$ was achieved for hard epoxy-embedded samples. A 3D extension of the conventional 2D LIBS imaging method was also developed, the applicability of which was tested both via volume reconstruction and direct depth-resolved analysis, ${ }^{269}$ working in a complementary way with conventional imaging techniques, i.e., transmission electron microscopy and fluorescence microscopy, ${ }^{266}$ studying the distribution of goldbased NPs in various organs such as the kidney, liver, and spleen. ${ }^{270}$

The first visualization of NP distribution in plant tissues with LIBS was published in 2017 by Czech researchers from Brno, who are still at the forefront of this research direction since then. In their first study, the distribution of Ag ions and ultrasmall Ag NPs in Vicia faba (faba bean) root cross-sections were detected with a resolution of $50 \mu \mathrm{m} .{ }^{271}$ In follow-up studies, Raphanus sativus L. (radish), Lemna minor L. (common duckweed), Sinapis alba L. (white mustard) were also used as test plants and the uptake and translocalization/accumulation of CdTe quantum dots, ${ }^{272,273}$ as well as $\mathrm{Y}$ - and Er-containing photon-upconversion fluorescent NPs. ${ }^{274}$ The studies revealed that the NP uptake rate is very slow and in certain plants there are differences in the way NPs and metal ions are accumulated. The obtained information was found to be very valuable in plant toxicity studies.

3.3.4. Photoacoustic spectroscopy. The most notable particle detection and characterization technique based on laser absorption is photoacoustic spectroscopy (PAS), which is used on particles dispersed in a gas. The general concept of the measurement is that if the wavelength of the laser light is suitable and it is absorbed by a particle, the temperature of the particle will quickly increase, and it starts to transfer the heat to the surrounding gas through the particle surface via conduction. This causes the surrounding gas to expand thereby a pressure wave is generated. This small pressure wave (pulse) can be picked up by a sensitive microphone, where the intensity of this signal will be proportional to the concentration and mass of the absorber (particles). This pressure signal is typically very small; therefore it is practical to modulate the power of the excitation laser beam (with an acoustic frequency) and have the aerosol sample in a cavity the dimensions of which are resonant 
with the wavelength of the modulation (length is a multiple of $\lambda$ / 2), placing the microphone at pressure nodes. Most acoustic resonators are cylindrical with radial or longitudinal plane wave modes being used. This way, a continuous excitation of the aerosol with the laser light will produce a larger, continuous pressure signal. By applying tunable or multiple wavelength laser sources, the quality or chemical composition of the particles can also be revealed..$^{39,275}$ Practical PAS instruments apply visible and MIR range semiconductor laser(s) as light source and are available commercially. ${ }^{276,277}$

It is essential in PAS that the release of heat from the particle occurs fast enough not to interfere with the modulation frequency, but this condition can be met easily for NPs, for which the temperature increase is typically on the order of millikelvins, and the thermal relaxation times are on the order of ns only. It is also generally important that the particle is dried before PAS measurements otherwise a loss of signal can occur due to mass transfer (release of volatile compounds); but again, this is less of a concern for NPs due to the tiny temperature increase. ${ }^{277}$

Unfortunately, the very small mass and $\Delta T$ of NPs also means that their PAS signal is very small, in fact it was shown to rapidly decrease with the particle size. ${ }^{278}$ Due to this reason, PAS is still far more commonly used for the characterization of microparticles rather than nanoparticles. However, using signal amplification (e.g. quartz-enhanced or optical cavity-enhanced PAS) and/or trapping by optical tweezers, the detection and characterization of nanodroplets ${ }^{279}$ and single NPs $(800 \mathrm{~nm}$ or less $)^{278,280,281}$ have been recently successfully demonstrated.

The one type of larger NPs (around or below $100 \mathrm{~nm}$ in diameter) that can be efficiently detected by PAS is soot or black carbon (these two names are often interchangeably used, see ref. 282), which have great importance in environmental science. ${ }^{280}$ In recent years, the internationally recognized PAS research group of Bozóki, Szabó and Ajtai has published several reports about successful method developments and applications for these atmospheric nanoaerosols (e.g. ref. 283-285).

3.3.5. Laser-induced fluorescence. Laser-induced fluorescence (LIF) is primarily used for the detection (e.g. counting, confirmation of presence, distribution monitoring) of fluorescent particles and chemical species in combustion analysis (planar laser-induced fluorescence, PLIF) ${ }^{286}$ and bioanalysis (e.g. laser flow cytometry, LFC).$^{287,288}$ Laser fluorescence detection of NPs can also be beneficially combined with laser scattering, e.g. NTA. ${ }^{289}$ Furthermore, fluorescent NPs can be employed for the labeling of various analytes to allow an easier detection. ${ }^{\mathbf{2 9 0 , 2 9 1}}$ For example, quantum dots are attractive fluorescence/Förster resonance energy transfer (FRET) donor chromophores for imaging and sensing purposes. ${ }^{292}$ It was even proposed that a combination of fluorescent polymer NPs should be used for the long-term labeling and tracking of living cells with any desired color code, as a proprietary "barcode" approach. ${ }^{293}$ LIF was also described as a potent tool for assessing the formation routes of NPs generated in laser ablation plumes ${ }^{294}$ or flame combustion processes $^{\mathbf{2 9 5}}$ (for details, please see Chapter 2.3.).

Particle characterization (e.g. identification, classification) style of applications of LIF, which fall more under the category of spectroscopy, are rare. One such awakening technique is LIFLIDAR (light detection and ranging). Absorption LIDAR for chemical analysis is a well-established long-distance stand-off laser spectroscopy method for atmospheric gas and aerosol analysis, which monitors the time-resolved attenuation of a laser pulse backscattered from distant aerosol particles occurring when the pulse traverses the space between the laser source and the particles. ${ }^{296}$ LIF-LIDAR is a variant of this technique, which uses the fluorescence emission signal of the aerosol particles for their detection and characterization. It has been recently demonstrated by a few publications that LIFLIDAR can classify atmospheric bioaerosol types (e.g. fungi, pollen, bacillus cells, etc.) based on their fluorescence emission spectra, even when their concentration in the air stream is as low as a few particles per liter. ${ }^{297-299}$ Obviously, both a low aerosol number concentration and a long optical pathlength (the latter due to the very small collection angle) work against the minimum detectable particle size. While most of the bioaerosol particles measured in these studies were in the tens of micrometer range, but some (e.g. bacillus cells) were only about $1 \mu \mathrm{m}$ in diameter, hence there is a promise that this approach can be developed into a nanoaerosol characterization technique soon.

3.3.6. Mass spectroscopy. Sorting and detection of chemical species based on their mass (or $\mathrm{m} / \mathrm{z}$ ratio) has become one of the most important detection principles in recent decades. Mass spectrometers are now increasingly used as standalone instruments or selective detectors and they also play a special role in the characterization of NPs. The reason for this is that mass spectroscopy (MS) is very sensitive, highly selective, it can provide elemental, isotopic, and molecular compositional information and can be coupled to other instrumentation (e.g. separation techniques, or sample introduction systems for gas/ liquid/solid phase analysis) relatively easily. These features make MS a valuable tool for NP characterization. Among others, the chemical composition, structure, porosity, ligand stoichiometries, size distribution, number concentrations of NPs can be determined by MS.

There are several publications that describe the application of MS to NP characterization. Excellent review papers on this topic are also available. ${ }^{\mathbf{3 0 0 - 3 0 2}}$ Although other approaches could have been also adopted for the classification of the related literature (e.g. according to the particle characteristics determined), but here we employed a four-tier, instrumentationoriented strategy. In the first, largest, section, we overview the progress in the so-called single particle ICP-MS (spICP-MS) subfield, which is perhaps the most versatile and has clearly produced the fastest growth in the past decade, with over several hundreds of papers in total. ${ }^{36}$ Naturally, this approach is primarily focusing on the analysis of engineered inorganic NPs dispersed in fluid media. The second sub-chapter gives an overview about the applications in which laser-ablation (LA) is used as a spatially resolved sample introduction approach for studies on the distribution of NPs in solid samples (similarly to LIBS-based approaches already discussed in Section 3.3.3.). In the third section, we briefly discuss the sub-field which relies on the combination of some separation techniques with ICP-MS. 
These allow for the analysis of polydisperse colloid systems and structurally complex nanosystems hardly accessible for other characterization approaches. Finally, some attention will be given to molecular (organic) MS applications too, which deal with the characterization of functionalized NPs (ligand coatings) or bio-nanoaerosols. Please note that, due to the scope of the present review, only the applications involving laser desorption/ionization (MALDI), or an ICP-MS detector will be discussed in the fourth chapter.

3.3.6.1. Single particle ICP-MS. Single particle ICP-MS (spICP-MS) is a novel technique capable for the investigation of individual nano- and microparticles in suspensions. The first application of spICP-MS was described in the articles of Degueldre et al. published ${ }^{303-306}$ between 2003 and 2006. In the early years, around 2010, the basic principles of spICP-MS (e.g. signal formation, data evaluation and calibration) was described by the research groups participating in early assessment of the capabilities of the technique..$^{307-309}$ By the end of that decade, spICP-MS has already become an established and rapidly developing NP characterization technique which enables the routine determination of the presence, size, size distribution and particle number concentration of dispersed NPs in an element- and isotope-selective way. The different aspects of spICP-MS were already assessed in detail in a handful of reviews. ${ }^{36,37,197,310,311}$ The following section presents only the basic principles of the technique, recent developments, and applications.

One of the greatest advantages of spICP-MS is that widespread ICP-MS instruments equipped with a (single) quadrupole mass analyzer are suitable for the characterization of individual NPs without any hardware or software modifications. NP suspensions can be analyzed directly by spICP-MS, hence the dissolution of the particles can be avoided, and the sample preparation generally includes only dilution and filtering steps. It should also be highlighted that spICP-MS can distinguish between the dissolved and the particulate form of the analyte. ${ }^{307,312}$ Thanks to the low particle number concentration sufficient for the analysis (approximately $10^{3}$ to $10^{5}$ particles per $\mathrm{mL}$ ), spICP-MS is a practical and convenient choice for the detection of NPs in environmental and biological media contrary to most other NP characterization methods. ${ }^{308}$

The basic concept of spICP-MS is that properly diluted NP suspensions are directly nebulized into the instrument, resulting in the presence of individual particles in separate droplets. Upon introduction into the plasma, these droplets/particles decompose (atomization and ionization) and the ion clouds of separate NPs are detected individually in time resolved analysis (TRA) mode. There are basically two possible approaches for the detection of single NPs by ICP-MS (Fig. 6). One - that was chronologically the first to be introduced into the literature utilizes a longer dwell time than the length of the TRA signal of the particles (that is typically a few hundred to thousand $\mu$ s (ref. 309 and 313-315)). Multiple times longer dwell times (e.g. 10 $\mathrm{ms})$, though, can result in the co-detection of more than one NP during the same dwell time. The probability of this can be minimized by the dilution of the sample suspension but the measurement time also needs to be increased to maintain the necessary number of detection events for a proper statistical evaluation. Co-detection of NPs can also be avoided by decreasing the applied dwell time (e.g. to less than 1-5 ms), but in this case, the probability of splitting the NP ion clouds randomly reaching the detector is increased (peak splitting) which has a negative effect on the accuracy of the particle size determination. It is further hindered by the signal loss due to detector settling time.

With the development of ICP-MS instruments, the utilization of dwell times shorter than the length of a particle event (high time resolution), e.g. 10-100 $\mu \mathrm{s}$, became available. During data evaluation, the split particle signals need to be assigned and integrated. The advantages of this approach are that it can reduce the possibility of particle co-detection, lower the continuous background intensity (resulting in a better signal/ noise ratio) and the possibility for the detection of multiple elements or isotopes in the same particle. ${ }^{317,318}$ On the other hand, the use of a lower dwell time also decreases the measured signals, which is critical for the quantitative measurement of NPs with a small size in the aspect of both the detection and the peak bordering. ${ }^{319}$

By performing the individual detection of NPs, a linear relationship can be drawn between the number of detected particle events $\left(n_{\mathrm{P}}\right)$ and the particle number concentration $\left(c_{\mathrm{P}}\right)$ :

$$
n_{\mathrm{P}}=\eta_{\mathrm{neb}} Q t_{\mathrm{dwell}} c_{\mathrm{P}}
$$

where $\eta_{\text {neb }}$ is the transport efficiency, $Q$ is the sample uptake rate and $t_{\mathrm{dwell}}$ is the dwell time. While $t_{\mathrm{dwell}}$ is a user-configured setting and $Q$ can be determined with relative ease, the accurate measurement of aerosol transport efficiency is more challenging as it is jointly affected by the components of the sample introduction system (nebulizer, spray chamber) and several experimental parameters (e.g. sample uptake rate and nebulizer gas flow rate). Pace et al. established three (waste collection, particle size, particle frequency) methods to determine this value. $^{308}$

For solid, homogeneous, spherical NPs, the correspondence between the intensity of a particle peak $\left(I_{\mathrm{P}}\right)$ and the mass of monitored analyte in the particle $\left(m_{\mathrm{P}}\right)$ can be described as:

$$
I_{\mathrm{P}}=K_{\mathrm{ICP}-\mathrm{MS}} \frac{A N_{\mathrm{Av}}}{M_{\mathrm{M}}} w_{\text {analyte }} m_{\mathrm{P}}
$$

where $K_{\text {ICP-MS }}$ is the detection efficiency (the ratio of the number of ions detected versus the number of atoms introduced into the plasma), $A$ is the atomic abundance of the monitored isotope, $N_{\mathrm{Av}}$ the Avogadro number, $M_{\mathrm{M}}$ the atomic mass of the element and $w_{\text {analyte }}$ is the ratio of the mass of the analyte and the particle (mass fraction). In the case of single component $\left(w_{\text {analyte }}=1\right)$ NPs, the signal of an individual particle at a particular $m / z$ is:

$$
I_{\mathrm{P}}=K_{\mathrm{ICP}-\mathrm{MS}} \frac{A N_{\mathrm{Av}}}{M_{\mathrm{M}}} \rho \frac{\pi d^{3}}{6}
$$

where $\rho$ is the density of the NP and $d$ is the particle diameter.

To determine NP size, size distribution and particle size detection limits, the calibration of spICP-MS is needed, during 

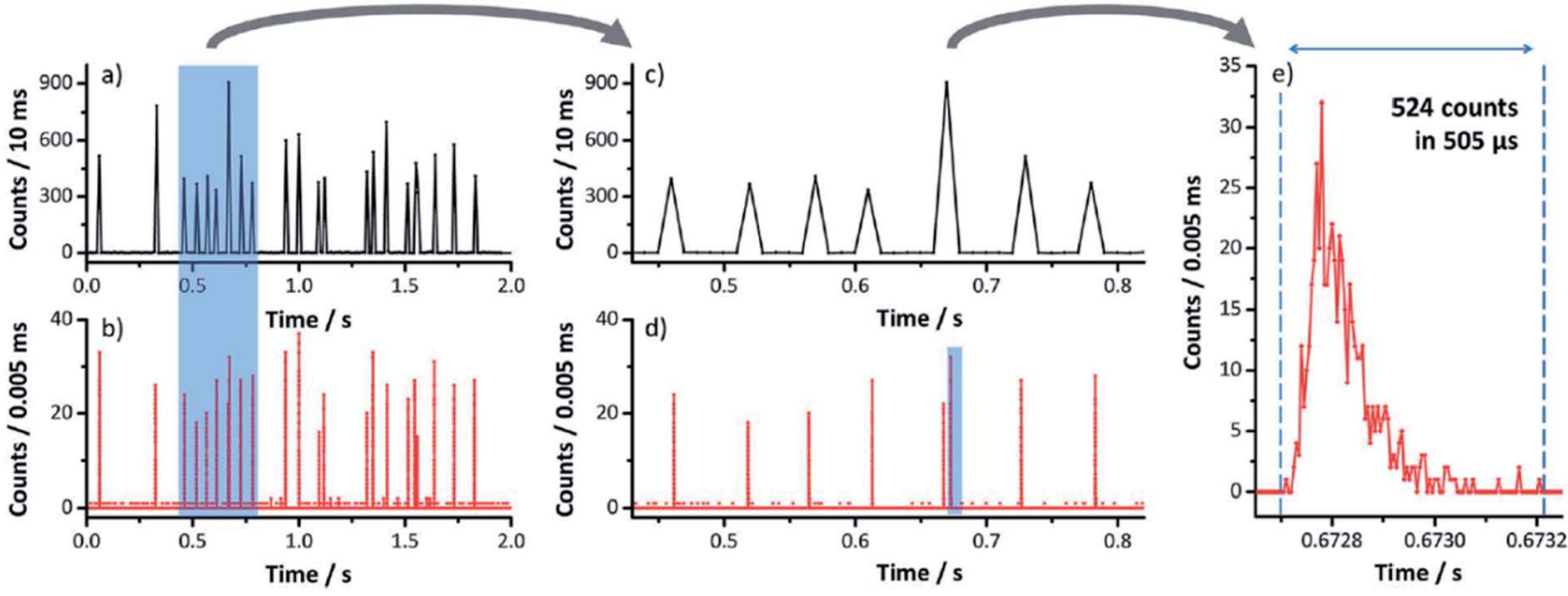

Fig. 6 Representative ICP-MS signal (monitoring $\mathrm{m} / \mathrm{z}^{197} \mathrm{Au}$ ) due to $30 \mathrm{~nm}$ AuNPs $\left(2.5 \times 10^{5} \mathrm{~mL}^{-1}\right.$ ) acquired simultaneously for $2 \mathrm{~s}$ with (a) $10 \mathrm{~ms}$ dwell time (vendor software), and (b) $5 \mu$ s dwell time (home-built data acquisition unit). First zoom level shows several particle events in (c) and (d) for about $400 \mathrm{~ms}$ (for the highlighted section in (a) and (b)). Second zoom level (e) shows the temporal profile of a single particle's ion cloud identified with the home-built data acquisition unit in (d). Adapted from ref. 316.

which process the function relating the particle mass to detected signals is established. In practice, spICP-MS calibration can be performed in two ways, utilizing (i) standard NP suspensions or (ii) standard solutions containing the investigated analyte. ${ }^{306,308}$ Although some earlier studies used a linear function to fit calibration data, but in principle, the calibration function follows a cubic relationship between the particle size (diameter or radius) and the ICP-MS signal. ${ }^{306}$ This logical assumption is also supported by recent plasma modeling studies which proved that practically the whole NP ion cloud passes through the hole of the sampling cone under typical experimental conditions. ${ }^{210}$ Although the utilization of NP standards for spICP-MS calibration purposes is straightforward and less exposed to the effects of experimental variability, a notable limiting factor is the shortage in the commercial availability of monodisperse, well-characterized particle standards.

The application of standard solutions for spICP-MS calibration was first introduced by Pace and co-workers. ${ }^{308}$ An essential condition to use this calibration technique is that after the decomposition in plasma, analyte ions with the same elemental composition go through the same (or reasonably similar) processes until their detection in the cases of both NPs and the solute. The mass of individual NPs is calculated on the basis of mass flow of the solution:

$$
W=c_{\text {std }} \eta_{\text {neb }} Q t_{\text {dwell }}
$$

where $W$ is the mass flow within a unit of dwell time, and $c_{\text {std }}$ is the mass concentration of the standard solution. Then, if the geometry of the particles is known, the size of the particles can be calculated.

In spICP-MS literature, the size detection limit is calculated as three or five times the standard deviation of the background signal. ${ }^{306,308,320}$ Size detection limits are typically around 10$40 \mathrm{~nm}$ for one component metallic NPs. ${ }^{36,321}$ There is also an upper limit for the NP size, as particles larger than a certain size do not spend enough time in the plasma for full decomposition and ionization. ${ }^{309,322}$ This upper size limit heavily depends on the studied element, but it can be estimated to be about a micrometer.

The main research goals related to spICP-MS sample introduction are to decrease the required sample volume and to achieve approximately $100 \%$ transport efficiency in order to obtain lower particle number detection limits. It also promotes calibration carried out using ionic standard solutions. Flow injection systems offer an attractive alternative for the introduction of NP suspensions. This is because by utilizing an injection loop of known volume, the total injected mass of analyte is also known, thus the determination of transport efficiency and sample uptake rate is not required for the measurement of the mass of NPs. ${ }^{323,324}$ Another possibility to achieve the highest possible transport efficiency is represented by microdroplet generator (MDG) sample introduction systems which can produce monodisperse droplets with a controlled volume and speed..$^{325,326}$ Unfortunately, MDGs are more prone to contamination and clogging compared to pneumatic nebulizers, hence, several papers demonstrated the advantageous utilization of the combination of pneumatic nebulizers and MDGs. ${ }^{327-330}$ In these setups, MDG provided well-defined droplets of known dissolved ion concentration for calibration purposes and NPs were introduced by a pneumatic nebulizer via a dual-inlet system.

It was also shown that - like solution mode ICP-MS - spICPMS is prone to certain interferences. The occurrence of nonspectral interferences in matrices containing carbon or sodium in high concentration can have a significant impact on NP signals, which unfavorably affects NP sizing. ${ }^{331,332}$ To tackle these undesirable effects, Telgmann et al. performed isotope dilution using ${ }^{109} \mathrm{Ag}$ enriched standard solution to characterize $\mathrm{Ag}$ NPs in wastewater and river water matrices. ${ }^{333}$ Huang et al. successfully applied dissolved $\mathrm{Rh}$ as internal standard to accurately determine the size distribution of polydisperse $\mathrm{CeO}_{2} \mathrm{NPs}$ 
in human urine, rat plasma and enzyme-digested rat liver tissue matrices that showed significant matrix effect. ${ }^{\mathbf{3 3 4}}$ The already described pneumatic nebulizer plus MDG dual-inlet sample introduction system offers another possibility to overcome nonspectral interferences as calibration solutions are exposed to the same matrix effects as the investigated NPs. ${ }^{328,335}$ Spectral interferences and the effect of collision/reaction cell (CRC) technology on NP signals were also investigated in the literature of spICP-MS. Kálomista et al. proved the usefulness of the application of $\mathrm{He}$ collision gas to eliminate polyatomic interferences for the analysis of $\mathrm{Cr}_{2} \mathrm{O}_{3}$ and $\mathrm{Fe}_{2} \mathrm{O}_{3}$ NPs. ${ }^{312}$ The advantageous application of a "triple-quadrupole" (tandem) ICP-MS with a CRC was demonstrated in several papers as well. Bolea-Fernandez et al. characterized the size of $\mathrm{SiO}_{2}$ particles using $\mathrm{H}_{2}$ reaction gas and on-mass analysis, and achieved a $75 \mathrm{~nm}$ size LOD. ${ }^{336}$ For the analysis of titania NPs Tharaud et al. NPs suggested the use of $\mathrm{NH}_{3}$ reaction gas in calcium-rich matrices, ${ }^{337}$ while Candás-Zapico et al. demonstrated the superiority of the application of $\mathrm{O}_{2}$ reaction gas to decrease size detection limits in the absence of $\mathrm{Ca}$ in the sample matrix. ${ }^{338}$ $\mathrm{Fe}_{3} \mathrm{O}_{4}$ NPs were characterized by Rua-Ibarz et al. using an onmass CRC technique using $\mathrm{H}_{2}$ and the authors developed a "pseudo resolution" sector field ICP-MS method for fast transient ion signal monitoring and to overcome the $\mathrm{ArO}^{+}$ spectral overlap with ${ }^{56} \mathrm{Fe} .{ }^{339}$

The multi-element detection capabilities of quadrupoleequipped ICP-MS instruments are limited due to their sequential principle of operation which also introduces a signal settling time when the mass analyzer is re-tuned. The detectable number of elements in a single particle is influenced by both instrumental parameters (e.g. gas flow rates, ${ }^{340}$ length of dwell time and settling time ${ }^{318}$ ) and NP properties (transient signal length through the particle size and the structure of the $\mathrm{NP}^{314}$ ). Even by utilizing $\mu$ s dwell times, usually no more than a 3-4 analyte isotopes can be determined within a particle and at some cost of quantitativity of the results. ${ }^{341}$ ICP-MS instruments equipped with a time-of-flight mass analyzer offer a promising approach for the quasi-simultaneous multielement analysis of NPs (spICP-TOFMS). Naasz et al. demonstrated the utility of spICP-TOFMS by the multielement (Fe, Mo, Cr, Ni) analysis of composite nano-steel platelets. ${ }^{342}$ Multi-element fingerprinting was also shown to help in the differentiation of geogenic and engineered NPs. ${ }^{341,343,344}$

The versatility of spICP-MS was further increased by research groups developing methodologies and data evaluation processes to determine additional important NP characteristics beyond particle size distribution and number concentration. Kálomista et al. utilized microsecond range data recording and demonstrated that by comparing the transit time of the ion cloud of particle events, spherical and rod particle geometries can be distinguished (Fig. 7), moreover, the aspect ratio (the length to width ratio) of nanorods can be determined. ${ }^{313}$ High time resolution data was also exploited to gain information on particle structure; Kéri et al. developed a data evaluation method to discriminate mixed alloy and core-shell $\mathrm{Au}-\mathrm{Ag} \mathrm{NP}$ transient signals. ${ }^{314}$ Completing single particle detection with the data of particle external diameter obtained by another NP characterization method (e.g. DLS, NTA, SEM, TEM), the density and porosity of NPs can also be calculated. ${ }^{345-347}$ Sápi et al. utilized spICP-MS to determine platinum concentration of Pt/ $\mathrm{SiO}_{2}$ nanocomposite decorated with ultra-small Pt NPs and highlighted the outstanding accuracy and precision of the spICP-MS technique by comparing its results to the ones obtained by other wide-spread NP characterization methods (TEM, solution mode ICP-MS, SEM-EDX, XPS). ${ }^{348}$

The excellent matrix tolerance and sensitivity of ICP-MS instruments and low particle number detection limits of the single particle technique enable the characterization of natural and engineered colloid particles in quite complex matrices too. Environmental applications include the analysis of NPs in lake, river and sea water, wastewater, soil as well as sediment samples. ${ }^{311,349}$ NPs were also detected and characterized in human tissues, ${ }^{350}$ urine $^{351-353}$ blood, ${ }^{352,353}$ serum $^{353}$ exhaled breath condensate, ${ }^{354}$ simulated gastric fluid ${ }^{355}$ and artificial sweat mixture, ${ }^{356}$ animal tissues and blood ${ }^{357-359}$ and in different parts of plants ${ }^{360-362}$ as well.

For risk assessment, the characterization of NPs in food products is an important task. NPs can accumulate in animals harvested as food from environmental sources. Zhou et al. applied spICP-MS for the characterization of metallic NPs in shellfish seafood and detected Y, La, Ce, Pr, Gd NPs in clams and Y, La, Ce, Pr, Nd NPs in oysters. ${ }^{363} \mathrm{Xu}$ et al. also analyzed NPs in marine mollusks from an offshore aquaculture farm and demonstrated the bioaccumulation of Ag containing NPs in the investigated organisms. ${ }^{364}$ NPs can also stem from food additives, e.g. E 171 colorants of different products were proved to contain $\mathrm{TiO}_{2}$ NPs with particle size below $100 \mathrm{~nm}$ in significant amount. ${ }^{365-367}$ Loeschner et al. demonstrated the presence of Alcontaining NPs in Chinese noodles, though, the origin of the particles (whether natural source, contamination of food storage/processing or additive agent) stayed unclear. ${ }^{368}$

The number of consumer goods that are intentionally fabricated with the addition of NPs to improve their attributes is also rapidly increasing. The potential of spICP-MS was demonstrated in the literature by the characterization of $\mathrm{TiO}_{2}$ NPs in sunscreens, ${ }^{369} \mathrm{Al}_{2} \mathrm{O}_{3}$ and $\mathrm{TiO}_{2}$ NPs in toothpaste ${ }^{370}$ and $\mathrm{Au}$ and $\mathrm{Ag}$ NPs in consumer spray products. ${ }^{371}$ Mackevica et al. proved the release of $\mathrm{Ag}$ NPs from toothbrushes, and $\mathrm{TiO}_{2} \mathrm{NPS}$ from textiles. ${ }^{372,373}$ Ntim et al. investigated $\mathrm{TiO}_{2}$ and $\mathrm{SiO}_{2} \mathrm{NP}$ migration from ceramic cookware to foodstuff. ${ }^{374}$ Several studies prove the usefulness of spICP-MS for the analysis of the particles present in or released from industrial products as well. The release of nano and sub-micron particles was monitored from different painted surfaces, ${ }^{375,376}$ printed nanosilver circuits $^{377}$ and nano-enabled pressure-treated lumber ${ }^{378}$ and their presence was demonstrated in tattoo inks $\mathrm{s}^{379,380}$ as well.

The exploitation of spICP-MS for bioanalytics is also advantageous. $^{381,382}$ For example, it was utilized for the individual detection of cells that is generally referred to as single cell ICPMS (scICP-MS) in the literature. As cells are significantly larger than NPs, special sample introduction tools were developed and applied for scICP-MS to boost cell transport efficiency. ${ }^{\mathbf{3 8 3}}$ The cell number concentration can be determined by the measurement of the (natural/spiked) major elements (e.g. P, Mg) of the 

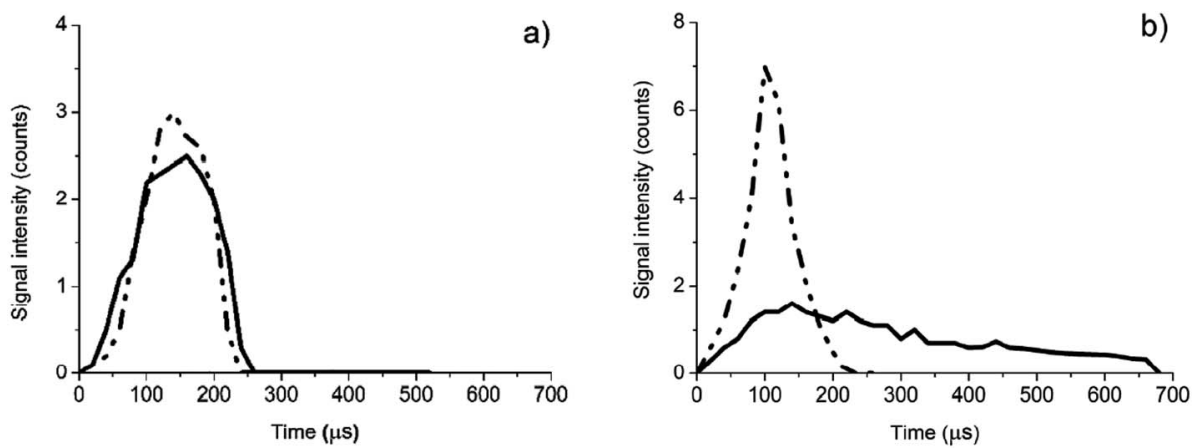

Fig. 7 A comparison of the high time resolution signal profiles for $30 \mathrm{~nm}$ diameter spherical gold NPs (panel a) and for 2.63 aspect ratio gold nanorods (panel b). In each panel, the two extreme time profiles (with the shortest (dashed dotted line) and longest (solid line) durations observed) as averages for $40-50$ particles are shown. ${ }^{313}$

cells $^{384,385}$ and information can be obtained on the presence, ${ }^{386,387}$ bioavailability ${ }^{388}$ and uptake kinetics ${ }^{389}$ of different compounds and particles related to the cells. spICP-MS can also be exploited as a sensitive readout tool for NP immunoassay tags, where the analytical information originates from the proportionality between the frequency of the detected particle events and the concentration of the analyte labelled with NPs. ${ }^{390-392}$

Besides the above-mentioned applications, there are other special fields where spICP-MS has already revealed its potential. For example, the characterization of metallic NPs in nonaquatic media was carried out by Nelson et al. who investigated asphaltene samples diluted in $o$-xylene. ${ }^{393}$ Ruhland et al. detected Hg-containing NPs dispersed in tetrahydrofuran that originate from gas condensates of petroleum hydrocarbon samples. ${ }^{394}$ It was also demonstrated that inorganic gunshot residue contains NPs that can be analyzed by spICP-MS for forensic purposes.$^{395}$ Fine (sub-micron and nano) particles were dispersed and detected by spICP-MS from electronic cigarette aerosols $\mathrm{s}^{396}$ and paper printing and shredding process ${ }^{397}$ as well. The technique was assessed as especially favorable for the fast and sensitive probe for functional pore sizes of ultrafiltration membranes by investigating the retention of NPs. ${ }^{398}$

3.3.6.2. LA-ICP-MS. The main focus in this LA-ICP-MS application field is the imaging of the spatial distribution of NPs within a solid material, which is often of biological origin. The frozen or epoxy embedded sample is placed on a three-axis stage and by scanning the laser beam across the sample surface, the material is ablated, and the resulting aerosol is transported with a carrier gas flow into the plasma. A polymer, glass or metal tubing is used as a transfer line. Argon, helium or rather their mixture is used as carrier gas as the addition of He was found to be beneficial from the point of view of lateral resolution (due to a lower amount of ablation debris) and of the formation of finer aerosol. The best spatial resolution achievable is about a couple of micrometers, which is suitable for the study of individual cells (e.g. NP uptake studies).

In case nanomaterials are present in the sample it is likely that their structure is significantly changed during the ablation and that signal will be generated from both the particles and the surrounding matrix. For this reason, most of the LA-ICP-MS NP studies work with NPs that are composed of elements not normally present, at a detectable concentration, in natural samples; hence mainly particles of gold, silver, platinum-group or rare-earth elements are monitored. It is also customary to use a lower laser fluence ( $c a .0 .1-1 \mathrm{~J} \mathrm{~cm}^{-2}$ ) in order to keep the disintegration of NPs to the minimum during laser ablation. It is worth mentioning that LA-ICP-MS imaging of NPs offers more analytical information than LIBS, although both methods are based on laser ablation. Interestingly, the reason for this advantage is caused by a feature of LA-ICP-MS, which is typically considered as a drawback in quantitative elemental analysis, namely the necessity for the transport of ablated matter from the location of ablation to the ICP plasma. This allows ICP-MS detection to be used somewhat similarly to spICP-MS for colloid systems, namely, to count individual NPs.

Various calibration approaches have been tested, but the signal response, at least in some applications, was also found to be depend on nanoparticle size, which seems to dictate that suitable calibration strategies and further fundamental studies on the behavior of NPs during laser ablation are needed to achieve reliable quantification. The spatial resolution and sensitivity are not yet sufficient to monitor smaller single nanoparticles in cells or tissues; typically, tens or hundreds of simultaneously ablated smaller, or individual several hundreds of nanometer NPs can be detected in the laser focal spot. More information about the above and other aspects can be found in recent LA-ICP-MS reviews. ${ }^{205,399-401}$

The following table gives a comprehensive overview of related NP studies. Please note that studies using NPs as elemental labels are not listed here, since NPs are not the direct object of those studies (Table 2).

In order to obtain complementary information about the molecular environment or fate of NPs in biological samples, LAICP-MS is sometimes combined with other laser-based analytical spectroscopies. For example, it was reported by Elci et al. that laser desorption-ionization MS (LDI-MS) images are specific for the ligands (surface functionalization) of NPs while they are still attached to the nanoparticle. Overlay of LDI-MS and LA-ICP-MS images allowed the authors to calculate the 
Table 2 An overview of LA-ICP-MS analysis of NPs in recent literature

\begin{tabular}{|c|c|c|c|c|c|}
\hline Detected nanoparticles & Sample type & Analytical goal & $\begin{array}{l}\text { Laser } \\
\text { wavelength }\end{array}$ & Spot size & Reference \\
\hline $\begin{array}{l}25 \mathrm{~nm} \mathrm{Au} \\
50 \mathrm{~nm} \mathrm{Ag}\end{array}$ & Fibroblast cells & $\begin{array}{l}\text { Quantitative imaging (calibration via } \\
\text { dried nanodispersion droplets on } \\
\text { nitrocellulose) }\end{array}$ & $213 \mathrm{~nm}$ & $4 \mu \mathrm{m}$ & 402 \\
\hline Au nanorods (aspect ratio: 4 ) & Human skin tissue & $\begin{array}{l}\text { Quantitative imaging (calibration via } \\
\text { spiked gelatine slices) }\end{array}$ & $213 \mathrm{~nm}$ & $10 \mu \mathrm{m}$ & 403 \\
\hline $\begin{array}{l}7 \text { or } 20 \mathrm{~nm} \mathrm{TiO}_{2} \\
50 \text { or } 75 \mathrm{~nm} \mathrm{Ag}\end{array}$ & Mouse neuroblastoma cells & $\begin{array}{l}\text { Quantitative imaging (calibration via } \\
\text { ablation of acid digested and dried } \\
\text { nanodispersion droplet) }\end{array}$ & $213 \mathrm{~nm}$ & $200 \mu \mathrm{m}$ & 404 \\
\hline $2 \mathrm{~nm}$ functionalized $\mathrm{Au}$ & Mouse spleen and liver tissue & Qualitative imaging & $213 \mathrm{~nm}$ & $50 \mu \mathrm{m}$ & $\begin{array}{l}405 \text { and } \\
406\end{array}$ \\
\hline $14 \mathrm{~nm} \mathrm{Au}$ & Mouse fibroblast cells & Quantitative imaging & $213 \mathrm{~nm}$ & $8 \mu \mathrm{m}$ & 407 \\
\hline $60 \mathrm{~nm} \mathrm{Ag}$ & Onion cells & Size distribution and mapping & $260 \mathrm{~nm}$ & $15 \mu \mathrm{m}$ & 408 \\
\hline $60 \mathrm{~nm} \mathrm{Au}$ & & & $193 \mathrm{~nm}$ & & \\
\hline $30-50 \mathrm{~nm} \mathrm{CeO}$ & Radish leaves & NP uptake, distribution, translocation & $213 \mathrm{~nm}$ & $250 \mu \mathrm{m}$ & 409 \\
\hline $50 \mathrm{~nm} \mathrm{Ag}$ & Rat lung tissue & $\begin{array}{l}\text { Quantitative imaging (calibration via } \\
\text { spiking frozen slices with standard } \\
\text { a solution) }\end{array}$ & $213 \mathrm{~nm}$ & $4 \mu \mathrm{m}$ & 410 \\
\hline $15-30 \mathrm{~nm} \mathrm{La}{ }_{2} \mathrm{O}_{3}$ & $\begin{array}{l}\text { Stems and leaves of Pfaffia } \\
\text { glomerata }\end{array}$ & $\begin{array}{l}\text { Quantitative imaging (calibration via } \\
\text { pressed La-soaked filter paper) }\end{array}$ & $266 \mathrm{~nm}$ & n.a. & 411 \\
\hline $20-100 \mathrm{~nm} \mathrm{Au}$ & Sunflower plant roots & Size distribution and mapping & $213 \mathrm{~nm}$ & $10-85 \mu \mathrm{m}$ & 412 \\
\hline $58 \mathrm{~nm}$ Y/FITC-doped Si & Sweet basil leaves & Qualitative mapping & n.a. & n.a. & 413 \\
\hline $20 \mathrm{~nm} \mathrm{Ag}$ & Mouse macrophage cells & $\begin{array}{l}\text { Quantitative imaging (calibration via } \\
\text { isotope dilution) }\end{array}$ & $213 \mathrm{~nm}$ & $60 \mu \mathrm{m}$ & 414 \\
\hline $\begin{array}{l}\text { Various size } \mathrm{Ag}, \mathrm{Au}, \mathrm{CuO} \text {, } \\
\mathrm{ZnO}\end{array}$ & Zebrafish embryos & Qualitative mapping & $266 \mathrm{~nm}$ & $50 \mu \mathrm{m}$ & 415 \\
\hline $20-250 \mathrm{~nm} \mathrm{Au}$ & Soil & $\begin{array}{l}\text { Size distribution and number } \\
\text { concentration }\end{array}$ & $193 \mathrm{~nm}$ & $100 \mu \mathrm{m}$ & 416 \\
\hline $40 \mathrm{~nm} \mathrm{Au}$ & Gelatin & $\begin{array}{l}\text { Validation of a simulation model for } \\
\text { NP sizing and counting }\end{array}$ & $193 \mathrm{~nm}$ & $10-80 \mu \mathrm{m}$ & 417 \\
\hline 60 or $80 \mathrm{~nm} \mathrm{Au}$ & Mouse liver tissue & $\begin{array}{l}\text { Size distribution and quantitative } \\
\text { imaging }\end{array}$ & $213 \mathrm{~nm}$ & $\begin{array}{l}100-200 \\
\mu \mathrm{m}\end{array}$ & 418 \\
\hline
\end{tabular}

percentage of intact surface-coated NPs. ${ }^{406}$ Büchner et al. combined LA-ICP-MS imaging of Au NP-exposed fibroblast cells with surface enhanced Raman scattering and succeeded in correlating the spatial distribution of the nanoparticles with their molecular nanoenvironment. ${ }^{407}$

In some additional new studies, the means for a more reliable NP quantitation, the Achilles's heel of LA-ICP-MS, were tested. One such novel approach is the so-called microwell trapping. The core concept of this approach is to use microfabricated pits in polymer substrates (e.g. polycarbonate or polydimethylsiloxane) with diameters smaller than the laser spot size, fill these pits with a microliter volume of standard solution and then dry these microdroplets in place. This trapping technique ensures a good localization of well dosed-out amounts of the analyte, ready for calibration purposes. Calibration via microwell trapping was successfully employed for the LA-ICP-MS analysis of $\mathrm{TiO}_{2}$ catalyst nanoparticles ${ }^{419}$ and $\mathrm{Ag}$ NPs in human bronchial epithelial cells. ${ }^{\mathbf{4 2 0}}$ Another external calibration approach is based on the use of microdroplets (microspheres). This latter method was demonstrated for the quantitative imaging of $\mathrm{Fe}_{2} \mathrm{O}_{3} \mathrm{NPs}^{421}$ and for the uptake of $\mathrm{Ag}$ NPs in macrophages. ${ }^{414}$

3.3.6.3. Separation techniques coupled to ICP-MS. The combination of separation techniques with ICP-MS provides a very efficient tool for engineered NP characterization in complex matrices. In this combination, the separation technique brings size and shape fractionation to the table while ICPMS adds excellent selectivity, sensitivity, and the possibility for quantitation. In the past few years, these NP applications are on the rise, producing dozens of papers per year. Below, we highlight some selected papers and research directions in this field.

Size-exclusion chromatography (SEC) was one of the first separation technique coupled to ICP-MS in 2011, with the purpose of creating a diagnostic tool to monitor CdSe and CdSe/ ZnS quantum dots and their bioconjugates. ${ }^{422}$ The SEC-ICP-MS combination was later also successfully applied to the separation of dissolved ions and their NPs (CdSe/ZnS quantum dots and Au NPs) in natural waters. ${ }^{423}$ Unfortunately, it was established that SEC suffers from unspecific surface adsorption on the stationary phase, and this limits the accuracy of quantitative measurements. ${ }^{\mathbf{4 2 4}}$ A further separation alternative is hydrodynamic chromatography (HDC), which works well for NPs in the 5 to $300 \mathrm{~nm}$ range, typically has less than 10 minutes run time and requires minimal sample pretreatment. HDC-ICP-MS has already been used for the characterization of $\mathrm{Ag}$ and $\mathrm{Au}$ NPs in an environmental matrix (sewage sludge supernatant) ${ }^{425}$ and was also demonstrated to be useful for the determination of metal mass fraction, size, and number concentration of Au NPs 
in drinking water. ${ }^{\mathbf{4 2 6}} \mathrm{Ag}$ NPs were also characterized by this hyphenated technique in blood and plasma samples. ${ }^{\mathbf{4 2 7}}$ Pitkänen et al. ${ }^{\mathbf{4 2 8}}$ compared the quantitative performance of HDC-ICP-MS and SEC-ICP-MS and found that the resolution of SEC is generally superior to that of HDC, in accordance with the kinetic theory of separations.

Capillary electrophoresis (CE) is a separation technique with no stationary phase; thus it is expected to be free from some of the problems associated with chromatography-ICP-MS combinations. First, micellar electrokinetic chromatography (MEKC), a variant of $\mathrm{CE}$, was tested in the literature as early as in $2014 .{ }^{429}$ Good recoveries and peak area precision were obtained which allowed the reproducible detection and separation of $50 \mathrm{~nm}$ sized Au NPs in a dietary supplement. CE-ICP-MS NPs application are more and more popular since then, especially in the bioanalytical field (e.g. monitoring of the speciation changes of gold-containing nanomaterials in cells). Among others, it has been employed to the study of Au NPs in cytosol, ${ }^{430}$ interaction of superparamagnetic iron oxide NPs with proteins, ${ }^{\mathbf{4 3 1}} \mathrm{Au}$ NPs and their protein conjugates, ${ }^{\mathbf{4 3 2}}$ Se NPs in human plasma ${ }^{\mathbf{4 3 3}}$ and $\mathrm{Ag}$ NPs in complex media (antibiotic and antiseptic liquids, wastewater, etc.). ${ }^{\mathbf{4 3 4}}$

Field-flow fractionation (FFF) separates NPs according to their hydrodynamic diameter and can be used in a range of $c a$. $1 \mathrm{~nm}$ to $50 \mu \mathrm{m}$. Separation is achieved in a narrow channel (without a stationary phase) where, to the laminar flow that carries the NPs, an orthogonally oriented force/interaction (separation field) is applied. The separation field can be induced either by a crossflow, centrifugal force, electrical or magnetic field as well as temperature gradient. The absence of the stationary phase largely eliminates undesired adsorptions (however adsorption on the membrane may still occur) and preserves the morphology of NPs. Thus, NPs and their conjugates as well as their aggregates can be separated in the same run. Although it can be used with other detectors as well (e.g. fluorescence spectroscopy), but coupling FFF with ICP-MS also allows elemental and isotopic speciation of NPs. ${ }^{435,436}$ Dual detector systems have also been described ${ }^{\mathbf{4 3 7}}$ as a potent tool for the analysis of NPs of different composition, but similar size. Additionally, it was also suggested to be used for the assessment of the efficiency of bioconjugation reactions. ${ }^{438}$

Today, FFF-ICP-MS is quickly becoming the most popular separation-ICP-MS combination. ${ }^{439}$ It has been reportedly used in a range of synthetic, biomonitoring and environmental analytical NP applications. As examples to the variety of applications, the analysis of $\mathrm{CdSe} / \mathrm{ZnS}$ core-shell quantum dots, ${ }^{\mathbf{4 4 0}}$ the assessment of the particle size distribution and concentration of $\mathrm{TiO}_{2}$ and $\mathrm{ZnO}$ nanoparticles in cream and spray sunscreens, ${ }^{\mathbf{4 4 1}}$ the qualitative and quantitative analysis of $\mathrm{Ag}$ and $\mathrm{Au}$ NPs in human urine, blood, and serum, ${ }^{353}$ or the determination of nanoparticulate fractions of $\mathrm{P}, \mathrm{Fe}, \mathrm{Al}$, and $\mathrm{C}$ in natural waters ${ }^{\mathbf{4 2}}$ have to be mentioned.

Finally, a novel instrumental combination which is not yet widespread but shows great promise should also be mentioned here. This is differential mobility analysis (DMA) coupled to ICP-MS. This can be directly employed to the study of NPS dispersed in gases, although most applications analyze aqueous nanodispersions by first converting them to nanoaerosols using electrospray. Not only the size characterization of spherical $\mathrm{Au}$ NPs, ${ }^{443}$ but also length and diameter evaluation of $\mathrm{Au}$ nanorods $^{444}$ and the quantitative analysis of dendron-conjugated cisplatin-complexed Au NPs ${ }^{\mathbf{4 4 5}}$ have been demonstrated.

3.3.6.4. Laser desorption and ionization MS. Matrix-assisted laser desorption/ionization (MALDI) is an extremely popular, soft ionization method that uses a laser as ionization source in mass spectroscopy (MALDI-MS). Typically, an organic matrix is used to assist the LDI process; the added organic matrix absorbs the laser pulse energy and donates a proton (or metal cation) to the investigated (bio)polymer analyte (e.g. proteins and peptides, carbohydrates, lipids, synthetic polymers, etc.). ${ }^{\mathbf{4 4 6}}$ Although both the matrix and the analyte in MALDI-MS are traditionally organic, but in the past 10-20 years, it was discovered that NPs have exploitable properties to improve the analytical performance of MALDI. For example, NPs can be tailored to have good light absorption at the laser wavelength, they have a high surface area which is available for analyte adsorption and if magnetic then the analyte collection and sample preparation is also easier. ${ }^{447-449}$

Nanoparticle-induced laser desorption ionization or NPtagging of molecules is not so relevant in the context of this chapter though, as NPs are not subject of the investigations. At the same time, applications to NP detection and characterization are quite rare. Aerosol mass spectrometers were constructed based on the LDI-MS principle for the specific goal of the detection or identification of individual airborne bioaerosol particles. After aerosol stream focusing and particle sizing, the bioaerosol particles are selected by monitoring their fluorescence activity, exploiting the fact that three canonical amino acids tryptophan, tyrosine, and phenylalanine (building blocks of peptides, proteins and therefore bacteria as well) are fluorescent. In most publications, the particle selection is based on tryptophan, which can be efficiently excited by the readily available $266 \mathrm{~nm}$ laser light. ${ }^{\mathbf{4 5 0 , 4 5 1}}$ Desorption and ionization of the biological particles are achieved by ns pulses of either excimer laser (308 $\mathrm{nm}$ ) or a frequency-quadrupled Nd:YAG laser (266 nm) which is followed by the recording of the mass spectrum. Since several of these studies were of the proof-of-concept type, matrix assistance sometimes was also used (e.g. in the form of sinapinic acid) via co-nebulization of the test particles. Submicron-range bioaerosol particles were successfully detected and identified by this approach, for example vegetative cells of Escherichia coli and Erwinia herbicola ${ }^{\mathbf{4 5 2}}$ or Bacillus subtilis var. niger. ${ }^{450}$ Pioneering works in this field were performed by Spengler et al. and others. ${ }^{\mathbf{4 5 3}}$

More recently, LDI-MS was also used for the study of the relative quantity of ligands attached to $\mathrm{Ag}$ and $\mathrm{Au} \mathrm{NP}$ surfaces $^{\mathbf{4 5 5 , 4 5 6}}$ or the ligand shell morphology on such NPs. ${ }^{457,458}$ These studies interpreted the detected noble metal-ligand clusters as being composed of surface noble metal atoms that had desorbed together with the ligands that were originally attached to them. The composition of the complexes was used to determine the composition of the ligand shell. These studies were later also extended to $\mathrm{Au}-\mathrm{Ag}$ bimetallic NPs. ${ }^{459}$ 


\section{Signal enhancement provided by nanoparticles}

\subsection{Importance, driving force and overview}

Plasmonics is the study of the interaction of light with collective oscillations of electrons in metals at a metal-dielectric interface. ${ }^{460}$ Plasmonics covers various aspects of surface plasmons, including propagating surface plasmons on metal layers and localized surface plasmons of metallic NPs towards realization of a variety of surface-plasmon-based devices. ${ }^{460}$ Signal enhancement by metallic NPs is widely used in many fields of modern sensing and sensorics. The main phenomenon behind this is the local interaction of plasmons (collective oscillations of free electrons) localized at surfaces of metallic structures with external electromagnetic field. Plasmonic materials are widely used in optical spectroscopy because they can remarkably enhance the sensitivity, specificity and extend the fields of application of optical detection methods. Surface Enhanced Raman Scattering (SERS), Surface Enhanced Fluorescence (SEF), Surface Plasmon Resonance (SPR) and Localized Surface Plasmon Resonance (LSPR) are just some of the widely used plasmonic based sensing techniques. Functionalization of plasmonic surfaces can be utilized to achieve highly specific detection with orthogonal sensing capabilities.

The first observation of surface plasmons is dated back to more than a hundred years and is related to the anomalous decrease in the intensity of light reflected by a metallic grating. ${ }^{461}$ The next milestone was the explanation of the color of metallic colloidal particles by electromagnetic theory of scattering and absorption of light by a spherical particle. ${ }^{462}$ The first optical method for exciting surface plasmons using a prism was demonstrated by Kretschmann, ${ }^{463}$ and this was the origin for the development of surface plasmon resonance sensing technology. SERS has been discovered in the 1970 s, ${ }^{464}$ followed by SEF in the $1980 \mathrm{~s}^{465}$ Starting from the $1990 \mathrm{~s}$, the field of plasmonics turned towards applications and began to penetrate various fields, including biological applications and medical diagnostics. Novel techniques have been discovered that are based on their utilization, like scanning near-field optical microscopy, ${ }^{466}$ tip-enhanced Raman spectroscopy, ${ }^{467}$ localized surface plasmon resonance spectroscopy, ${ }^{468} \mathrm{NP}$-enhanced laserinduced breakdown spectroscopy, ${ }^{469}$ etc. This was accompanied by rapid development of chemical and physical methods for NP fabrication, ${ }^{470}$ targeted alteration of their properties and surface functionalization, ${ }^{471,472}$ together with coherent and broadband light sources, spectrometers, and detectors.

Nanoparticles, and especially metal NPs are amongst the agents for plasmon-based detection of highest efficiency. Metal NPs have special optical properties in comparison with their bulk forms. For example, silver and gold NPs exhibit strong absorption in the visible region, and copper NPs in the near infrared. The optical properties of NPs depend on their dimensions, shapes, composition, as well as the optical properties of the surrounding medium. ${ }^{473-476}$ NPs can be considered as complex multi-electron systems in which the electronic motion is confined, limited by the dimensions of the particle, leading to the occurrence of novel phenomena and effects, especially when the NPs size is much smaller than the wavelength of the light they are interacting with. In addition, interaction of NPs with light can be easily controlled through the material, geometry, aggregation, etc. The ease of surface functionalization is another advantage allowing to develop tailored and highly selective applications based on surface plasmons and NPs.

This chapter summarizes the main spectroscopic methods involving nanoparticles to increase their sensitivity, including LSPR, SERS, nanoparticle-enhanced laser-induced breakdown spectroscopy and inductively coupled plasma mass spectrometry (NELIBS and NE-ICPMS) and SEF. Different and multiple NP-related enhancement mechanisms are involved in these techniques, however, the localized surface plasmons excited in the NPs play crucial role in all of them. Therefore, the chapter begins with an introduction to propagating and localized surface plasmons and their properties, and the plasmonic efficiency of different metals. This is followed by the description of the above-described NP enhanced (or surface enhanced) methods, including their principle of operation, properties and some applications.

\subsection{A concise introduction to surface plasmon resonance}

Metals contain free conduction electrons and also interband transition electrons. These two groups of charged carriers determine the complex dielectric function (permittivity and permeability), that describes the optical properties of metals. For example, the combination of plasmon frequency described below and the character of the interband transitions gives metals their characteristic color. When light interacts with the metal, under certain circumstances it can excite collective motions of free electrons, the so called plasmons. They will be influenced by a time-dependent force opposite that of the changing electromagnetic field of the incident light and this will result in an oscillatory motion of the electrons, but $180^{\circ}$ out of phase. Like all oscillators, these electrons will also have a characteristic frequency, known as the plasmon frequency, being dependent on the density of electrons $(n)$ and their effective mass $\left(m_{\text {eff }}\right): 477$

$$
\omega_{\mathrm{p}}=\sqrt{\frac{n e^{2}}{m_{\mathrm{eff}} \varepsilon_{0}}}
$$

Here $e$ is the charge of the electron and $\varepsilon_{0}$ is the permittivity of the free space. If the frequency of the incident photon is above the plasmon frequency (in most cases this is true with UV photons), the light will be transmitted or absorbed by the interband electrons, and free electron oscillations will not occur. Photons with frequency below $\omega_{\mathrm{p}}$, on the other hand, will excite oscillations and will be reflected by the metal. Since the electric field of the incident light cannot penetrate the bulk of the metal deeper than the skin depth, ${ }^{477}$ it will excite plasmons at the metal surface, involving only a minority of the free electrons. Now, if the bulk metal is shrunk to a thin film, the oscillations will extend over the whole thickness, leading to propagating charge waves known as surface plasmon polaritons 
(SPP). ${ }^{478}$ Here "polariton" means a hybrid excitation, occurring when a surface plasmon couples with a photon, which situation generates charge waves travelling on the surface. ${ }^{479}$ Additional constraints are set on the frequencies with which the free electrons in metals can oscillate in the incident field by the interface between the metal film and the surrounding medium, which limits the continuous spectrum to a fixed wave vector and frequency for a given interface. The dispersion curve of SPP excitation can be described as: ${ }^{478}$

$$
k_{\mathrm{SPP}}(\omega)=\frac{\omega}{c} \sqrt{\frac{\varepsilon_{\text {metal }} \varepsilon_{\text {diel }}}{\varepsilon_{\text {metal }}+\varepsilon_{\text {diel }}}},
$$

where $k_{\mathrm{SPP}}$ is the wave vector of light necessary to excite a SPP for a given interface, $\varepsilon_{\text {metal }}$ and $\varepsilon_{\text {diel }}$ are the permittivities of the metal and the surrounding medium, respectively. Since the wave vector (or momentum) of the oscillating charge wave is always greater than that of the massless photon, SPP cannot be excited directly by incident light, but only by a prism in the Kretschmann geometry, or by a grating to supply the extra momentum. ${ }^{478}$ The angle for which the grating or the prism can supply the necessary momentum to excite the SPP can be determined from the dispersion curve $k_{\mathrm{SPP}}(\omega)$ (eqn (11)). At this angle, light will be absorbed, leading to a dip in the reflection or transmission spectrum. In addition to the properties of the metal, the dispersion is also influenced by those of the surrounding medium. Therefore, changes in the permittivity of the local environment will affect the excitation of SPP and can be used for sensing.

The suitability of a certain metal for plasmonic applications and plasmon resonance can be determined from its dielectric function. In the frames of the Drude model, the frequencydependent real and imaginary parts of the dielectric function can be described as:

$$
\begin{gathered}
\operatorname{Re}[\varepsilon(\omega)]=\varepsilon_{\infty}\left(1-\frac{\omega_{\mathrm{p}}{ }^{2}}{\omega^{2}-\tau_{0}^{2}}\right) \\
\operatorname{Im}[\varepsilon(\omega)]=\frac{\varepsilon_{\infty} \omega_{\mathrm{p}}{ }^{2} \tau_{0}}{\omega\left(\omega^{2}-\tau_{0}^{2}\right)}
\end{gathered}
$$

where $\varepsilon_{\infty}$ is the dielectric constant at infinitely high frequencies, $\tau_{0}$ is the collision frequency of electrons with ions and impurities in the structure. Taking into account that $\tau_{0} \ll \omega$, the plasmon frequency can be determined at $\operatorname{Re}(\varepsilon(\omega)) \approx 0$. It can also be seen that for the case of $\omega<\omega_{\mathrm{p}}$ the real part of the expression will be negative $(\operatorname{Re}[\varepsilon(\omega)]<0)$. In addition, if $\omega$ is not small, $\operatorname{Im}[\varepsilon(\omega)]$ will have small values. These two conclusions the real part of the dielectric function is negative and has a relatively large magnitude and the imaginary part is small are the main conditions of plasmonic resonance at a certain frequency to occur in bulk materials.

Analysis of the wavelength dependence of the real and imaginary parts of the dielectric function for different metals ${ }^{\mathbf{4 8 0}}$ shows that the real part of their dielectric function is negative in the Vis-NIR region. In the visible region aluminum has the highest absolute value, being close to 30 at $400 \mathrm{~nm}$ and increasing rapidly with the wavelength. It is followed by a group of three metals behaving very similarly - silver, gold, and copper. Their values are small up to $600 \mathrm{~nm}$ but increase afterwards. This group is followed by palladium, platinum, and lithium, having noticeable values in the red and near infrared wavelength regions. ${ }^{480}$ In terms of the imaginary part of the dielectric function, silver has the smallest value in the visible region. It is around 0.6 at $400 \mathrm{~nm}$ and increases nearly linearly to 1.7 at $800 \mathrm{~nm}$. Slightly higher values can be seen for lithium with similar wavelength dependence. Gold and copper are $>5$ at $400 \mathrm{~nm}$, but then they decrease and get close to silver at $650 \mathrm{~nm}$, from where the three follow the same trend. From the above it can be concluded that copper, gold, and silver are the materials with most favorable dielectric properties for efficient plasmon resonance. And indeed, silver and gold are the most widespread plasmonic materials used in SPR, SERS and other spectroscopic techniques nowadays.

The plasmonic conditions set for the real and imaginary parts of the dielectric function can be combined into the socalled quality factor as:

$$
Q=\frac{\omega\left(\frac{\mathrm{d}\{\operatorname{Re}[\varepsilon(\omega)]\}}{\mathrm{d} \omega}\right)}{2(\operatorname{Im}[\varepsilon(\omega)])^{2}}
$$

From eqn (13) it can be seen that the higher the rate of the change of the real part and the smaller the imaginary part of the dielectric function with frequency, the higher the quality factor is and the better the given material for plasmonic applications in the given wavelength region.

As eqn (11) shows, the excitation of SPP depends on the properties of both the metal layer and its environment. This relationship makes the excitation of surface plasmon resonance an outstanding tool for sensing, in which the changes in the dielectric properties of the local environment of the metal layer can be detected by measuring change in the resonance frequency (or the coupling angle) of surface plasmon polaritons. This is the basis of the SPR technique.

The $\omega_{\text {SPR }}$ SPP resonance frequency is determined by the plasmon frequency of the metal and the dielectric properties of the surrounding medium: ${ }^{478}$

$$
\omega_{\mathrm{SPR}}=\frac{\omega_{\mathrm{p}}}{\sqrt{1+\varepsilon_{\mathrm{diel}}}}
$$

The local electromagnetic field resulting from the charge oscillations during SPR extends a few hundreds of nanometers from the metal surface. So, if the local environment changes within this distance, the dielectric constant will differ, and the SPR frequency will shift. The narrow resonant line shape and high angular specificity of SPR allow excellent signal-to-noise ratio and figure of merit to be obtained for SPR-based sensors, but the measurement requires inpractically strict conditions and complex geometries. ${ }^{478}$

The strict conditions are not required when surface plasmons are excited in metal NPs instead of thin layers. In this case, if the NP size is below the wavelength of the incident light, the electric field will be constant across the NP, inducing 
a uniform displacement of the electron density and a strong restoring force from the positive ionic core background. ${ }^{\mathbf{4 8 1}}$ This interaction leads to collective oscillation of the free electron cloud of the NP with a characteristic oscillation resonance frequency. This phenomenon is known as localized surface plasmon resonance, or LSPR. There is no angular requirement in LSPR excitation, since the additional momentum is provided by the geometry of the NPs. ${ }^{\mathbf{4 8 1}}$ Therefore, the change in the LSPR wavelength can be detected without gratings or prisms, by using a simple spectrometer.

Similarly to SPR, the resonance frequency of LSPR also depends on the plasmon frequency of the metal and the dielectric function of the local environment of the NP: ${ }^{474}$

$$
\omega_{\mathrm{LSPR}}=\frac{\omega_{\mathrm{p}}}{\sqrt{1+2 \varepsilon_{\mathrm{diel}}}} .
$$

The main difference between $\omega_{\mathrm{SPR}}$ and $\omega_{\mathrm{LSPR}}$ is the factor 2 in the nominator, resulting in different resonance frequencies for the same metal-dielectric combinations. However, there are several advantages related to the use of quasi zero-dimensional NPs instead of two-dimensional thin layers for LSPR. ${ }^{474}$ The confined electron oscillations in LSPR cause intense local electromagnetic fields, which can be several orders of magnitude stronger than the incident field. This phenomenon is behind the concept of, for example, surface-enhanced Raman scattering. This field will be more concentrated around the edges in NPs with sharp edges, further increasing the local field intensity. It has been shown that the LSPR peak position is affected also by the size of the NPs. ${ }^{474}$ In general, the larger the particles, the more red-shifted the LSPR wavelength will be, ${ }^{\mathbf{4 7 4 4 8 2}}$ which can be leveraged for fine-tuning the plasmonic properties of NPs. Specific NPs with asymmetric shape (e.g. nanorods) have two LSPR peaks corresponding to their longitudinal and transversal oscillation modes, ${ }^{\mathbf{4 8 3}}$ and their separation can be tailored by the diameter and the size aspect ratio. Also, the electromagnetic field in LSPR decays in a few tens of nanometers and it is more sensitive to changes in the distance from the surface of the metal. ${ }^{\mathbf{4 7 4 , 4 7}}$ Another specific feature of NP-based LSPR is the coupling of local fields when the NPs are brought within the local field decay length. ${ }^{478}$ This coupling can enhance the local field intensity and shift the LSPR peak position due to hybridization of modes. ${ }^{474}$

\subsection{Methodology and applications}

Localized surface plasmons and plasmonic enhancement are used in many spectroscopic techniques nowadays. The simplest, yet highly efficient method is LSPR, in which metallic NPs are used as sensor transducers, the plasmon resonance frequency of which shifts when the dielectric properties of their local environment change. SERS and SEF utilize the resonant amplification of the electromagnetic field of the incident or emitted light through their interaction with strongly confined plasmons of NPs. In addition to LSPR, other effects also contribute to SEF, NELIBS and NE-LA-ICPMS. The following paragraphs summarize the main principles and some of the applications of these methods.
4.3.1. Localized surface plasmon resonance. The LSPR technique was developed based on SPR sensing. The latter means the detection of the interaction of propagating surface plasmons excited on a planar metal-dielectric interface with light of appropriate incident angle and/or wavelength. As it has been described earlier, these surface plasmons are extremely sensitive to the changes in the local environment of the boundary, such as to the adsorption of molecules to the metallic surface, which causes the shift of the optimal incident angle and/or the resonance wavelength. The SPR measurement is accomplished by scanning the angle of incidence at a fixed wavelength or by using a broad light source with multiple wavelengths at a fixed angle of incidence (in the so-called Kretschmann configuration). When the resonant conditions are met a dip in the angle or wavelength dependent reflectivity is experienced.

LSPR utilizes the same phenomenon, but on the boundaries of metallic NPs and their surrounding medium. Changes in the local environment are detected through the shift of the LSPR maximum wavelength of the broadband light transmitted through or reflected from the medium with NPs. The resonant frequency of NPs depends on their composition, size, geometry, dielectric environment, and separation distance. ${ }^{\mathbf{4 8 4 , 4 8 5}}$ Since many LSPR methods involve ensembles of nanoparticles that have some size distribution, the measured spectral signals are averaged quantities and can exhibit heterogeneous broadening.

In contrast to SPR, LSPR does not require complex optics. Since the conditions for resonance are simpler than those of SPR, the LSPR instrumentation can consist of a white light source and a spectrometer. Optical fibers can be used to couple the incident and transmitted/reflected light to/from the metallic NPs, making the LSPR experiments and development of LSPR systems extremely flexible and allowing to perform remote measurements as well. LSPR sensors have a greater potential for miniaturization and portability and are simple to integrate with microfluidics. ${ }^{486}$

Mostly $\mathrm{Au}, \mathrm{Ag}$ and $\mathrm{Cu}$ NPs are used in LSPR applications. These metal NPs exhibit shape and size dependent LSPR absorption and scattering bands, which is utilized to construct plasmonic sensors. They can be used as LSPR sensor transducers in three main configurations: in free-standing colloidal (homogeneous), surface-confined (heterogeneous) single NP and surface-confined (heterogeneous) NP array forms. ${ }^{\mathbf{4 8 6}}$

There are two types of NP-based plasmonic interactions causing LSPR peak shift. ${ }^{474}$ In the first type the LSPR wavelength shifts when an analyte binds to the NPs surface and changes the local refractive index, while in the second, the plasmonic fields of several nanoparticles are coupled when an analyte brings them into proximity (aggregation), causing a remarkable shift of the LSPR wavelength and thereby a color change. ${ }^{474}$ The local field enhancement can reach a factor of $10^{4}-10^{6}$ with separated metal NPs, while for colloidal aggregates ${ }^{487}$ it can be as high as $10^{14}$. Because of their large enhancement factors, colloidal aggregated NPs have been used in a variety of plasmonic applications including solar energy conversion, photocatalysis, nanomedicine, biological sensing, etc. ${ }^{487}$ 
Applications of LSPR for sensing cover many areas of modern technology, including gas and $\mathrm{pH}$ sensing, biology, medical diagnostics, or environmental protection. Several excellent review papers are available covering different fields of LSPR applications, including chemical analysis ${ }^{\mathbf{4 8 1}}$ and especially biosensing. ${ }^{\mathbf{4 8 1}, \mathbf{4 8 6 , 4 8 8 - 4 9 1}}$ While most of the NP-based LSPR sensors operate in liquid environment, there are developments targeting gas sensing in air, including hydrogen, ${ }^{\mathbf{4 9 2 , 4 9 3}}$ helium and argon, ${ }^{494}$ water vapor ${ }^{495}$ and volatile organic compounds. ${ }^{496}$ The simplest LSPR-based applications are based on the simple, fast and cheap colorimetric principle. ${ }^{497}$ Such methods have been developed for the detection of a variety of compounds, including metal ions, ${ }^{498}$ small organic molecules, ${ }^{499}$ proteins $^{500,501}$ and DNA. ${ }^{502,503}$

A novel LSPR based approach is based on the combination of plasmonic nanostructures with other sensing methods, such as responsive photonic crystals $(\mathrm{PCs})^{\mathbf{2 3 3 , 5 0 4 - 5 0 6}}$ or metal oxide films. ${ }^{507,508}$ PCs are optical materials consisting of periodically arranged materials with different dielectric constants. These structures can be used as sensing materials since their diffraction wavelengths or intensities will change when they are exposed to physical or chemical stimuli. ${ }^{233}$ PCs are widely used in detection of ions ${ }^{509}$ and gases, ${ }^{510}$ and also of electric ${ }^{511}$ and magnetic fields. ${ }^{512}$ Their response can be enhanced by plasmonic NPs, ${ }^{233,505}$ and that, in addition to the amplification of the signal coming from the PC, can also be used to immobilize the recognition element, providing specificity to the sensor. Combination of opal PCs with $\mathrm{SiO}_{2}-\mathrm{Au}$ NPs resulted in three orders of magnitude increase in the limit of detection of the PC has been observed after combining it with the plasmonic gold nanoparticles. ${ }^{233}$ The capabilities of this sensing platform were demonstrated with atrazine analogues, demonstrating a sensitivity of $10-12 \mathrm{~g} \mathrm{~mL}^{-1}$.

The sensitivity of gas sensing metal oxide films was also improved by combination with plasmonic NPs. Au:CuO composites were used to develop a high-resolution LSPR spectroscopy system for the detection of pure $\mathrm{Ar}, \mathrm{N}_{2}, \mathrm{O}_{2}$ gases. ${ }^{508}$ The composite structure was produced by sputtering a copper target with gold pellets on its surface, followed by a heat treatment promoting Au NP formation. A surface plasma treatment was used to activate the film for the detection of gas molecules. A one order of magnitude increase of the sensitivity was achieved, compared to the performance of copper oxide without the NPs. ${ }^{508}$

An emerging field of LSPR application is the sensing in the near-infrared (NIR) region, especially in at the wavelengths used in telecommunications (1530-1625 nm). Combination of the cheap and mature optical fiber technology with chemical and biological LSPR sensing leads to numerous new applications, especially in remote sensing technologies. As it has been discussed earlier, the metals useful for plasmonics must have a negative and high magnitude real and a small imaginary dielectric function, implying a large $Q$-factor. $\mathrm{Au}$ and $\mathrm{Cu}$ are the only metals meeting these criteria in the NIR region and indeed, NPs prepared from these materials showed strong absorption peaks there. ${ }^{513}$ Wide-bandgap semiconductors, like tin doled indium oxide, aluminum doped zinc oxide or titanium oxide $^{514-516}$ and transition-metal oxides (molybdenum oxide, ${ }^{517}$ tungsten oxide, ${ }^{518}$ vanadium oxide ${ }^{519}$ ) were also found to be good candidates for NIR plasmonics. Composite NPs of gold and molybdenum oxide were used for the detection of hydrogen. ${ }^{517}$ These NPs showed strong LSPR absorption in the NIR, being responsive to hydrogen, the presence of which caused increase and shift of the plasmonic peak. ${ }^{517}$

A new method for tuning the LSPR properties can be achieved by fabricating magnetic LSPR NPs and nanostructures, allowing to change the optical properties by the application of a magnetic field, as well as to modulate the magnetic properties under irradiation at the plasmon resonance. ${ }^{520}$ NPs with combined magnetic and LSPR properties were synthesized first from bimetallic structures such as $\mathrm{Ni}-\mathrm{Au}^{521}$ or $\mathrm{Co}-\mathrm{Au},{ }^{522}$ however, the performance was found to be strongly dependent on the dimensions, shape and positioning of the different components, making the reproducible preparation a main issue. ${ }^{520}$ Recently, arrays of periodically placed cobalt NPs were found to have plasmonic properties and a quality factor comparable to that of Au NPs. ${ }^{520}$ Particle size, shape and the interparticle distance were found to have a significant impact on the optical response here as well, however, the use of an intrinsically magnetic, single metal structure makes their control remarkably easier. The LSPR absorption peak of Co NP arrays extends above the visible region making them promising for NIR LSPR applications.

4.3.2. Surface enhanced Raman spectroscopy. Raman scattering is an inelastic light scattering process involving interaction of the incident photon with fundamental vibration of the molecule, so that the energy difference of the incident and scattered photons is equal to the energy of the involved vibration. Raman spectroscopy allows to record a fingerprint spectrum of the sample, characterizing its bonding configuration and consisting of peaks related to its possible vibrations, the excitation of which is allowed by the Raman process. ${ }^{523,524}$ Raman spectra are recorded by illuminating the sample with a monochromatic light source and recording the spectrum of the scattered light in the wavelength region different from that of the excitation source. In general, the position of the Raman peaks in the spectrum is independent of the energy of the excitation photons, however, if the latter is equal to the energy of an existing electronic transition of the material, resonant Raman scattering occurs, the efficiency of which is a few orders of magnitude larger than that of the normal Raman process. ${ }^{523}$

Besides resonant Raman scattering, surface enhanced Raman scattering (SERS) is another phenomenon causing the enhancement of the Raman signal. SERS was first discovered in the 1970 s by recording an unexpectedly large Raman signal when measuring pyridine adsorbed on a roughened silver electrode. ${ }^{464}$ The first explanations were attributing this phenomenon to the formation of strong electrochemical electromagnetic fields or molecular-metal complexes on the metal surface, ${ }^{524,525}$ as well as to the characteristics of the nanostructured metal surface and the optical excitation of the collective oscillation of electrons. ${ }^{526,527}$ The research and especially applications of SERS started to emerge in the 1990s, when, thanks to the development of compact coherent light sources 
and spectroscopic instrumentation, Raman spectrometers appeared in more and more spectroscopic and analytical chemistry laboratories. SERS was found to offer simple, rapid, non-invasive, and non-destructive analytical capability providing information about the bonding configuration of target molecules. ${ }^{528}$ Its proven ability for single-molecule detection drives the development of different SERS enhancement platforms for the detection of molecules on a sub-ppb or even lower levels. ${ }^{529}$ As the SERS spectrum of water is rather weak, ${ }^{530}$ SERS can efficiently be used to study biological samples and to detect biomolecules even at very low concentrations. ${ }^{531}$ SERS amplification was also observed with dielectrics and semiconductors, but with a much smaller enhancement than with metal based plasmonic structures. ${ }^{532}$

While the SERS enhancement mechanism is not completely understood yet, it is generally accepted that there are two enhancement mechanisms contributing to SERS: the so-called electromagnetic $^{\mathbf{4 8 0 , 5 3 3}}$ and the chemical enhancement. ${ }^{534}$ The former is based on the interaction of the incident and/or scattered light with localized surface plasmons, resulting in amplification of their electromagnetic field and the Raman scattering on molecules being in close vicinity of the plasmonic surface. This mechanism can provide SERS enhancement on the level of 6-9 orders of magnitude. ${ }^{535}$ Chemical enhancement is the result of complex formation between the plasmonic surface and the molecule, sometimes accompanied by generation of new electron energy levels, involved in resonant Raman scattering. In this regard chemical enhancement is not a plasmonic process but rather a specific Raman scattering in which the energy difference of the newly created energy levels is equal to the energy of the exciting photons. A 2-4 orders of magnitude of SERS enhancement can be attributed to this mechanism. ${ }^{\mathbf{5 3 5}}$

The electromagnetic SERS enhancement is independent of the type of the molecule, but strongly depends on the properties of the plasmonic surface, including its material, size, shape and, in case of SERS substrates, surface roughness. ${ }^{536}$ Since Raman scattering involves photons of two different wavelengths, the electromagnetic enhancement has two distinct contributions. The first is the near-field enhancement, during which the LSPR excitation induces strong spatial localization and amplification of the excitation laser light in small spatial regions, called hotspots. ${ }^{536}$ Around the hotspots the molecules experience a much stronger electromagnetic field than in the normal laser radiation, resulting in high levels of Raman enhancement. The second is the re-radiation enhancement related to the amplification of the Raman scattered light radiated by the molecule, that arises from the environmentdependent emission by the Raman scattering molecule, as a dipole, affected by the interface of the plasmonic surface, sometimes referred as modified spontaneous emission. ${ }^{536}$ While a dipole (molecule) has a typical, symmetric emission pattern in vacuum or homogeneous medium, its irradiated electromagnetic field will be scattered at nearby interfaces and will be partly back-scattered to the dipole. This back-scattered radiation (or self-reaction field) will influence the way in which the dipole radiates power and could contribute to the enhancement of Raman scattering. ${ }^{536}$
The chemical SERS enhancement is related to the adsorption of the molecule on the plasmonic surface, accompanied by bond formation. This could contribute to the increased SRS signal in two different ways. During the resonant charge transfer the interaction between the molecule and the metal results in the creation of a metal-molecule charge transfer state, involved in resonant Raman scattering process, resulting in remarkably enhanced Raman intensity. ${ }^{536}$ In the non-resonant chemical effect, there are no new electronic states involving the resonant process, but the interaction induces changes in the geometry and electronic structure of the molecule, affecting the probability of certain Raman transitions and resulting in enhanced Raman intensities, but also slight modification of the Raman peak positions. ${ }^{536}$

From the practical point of view, SERS requires the same instrumentation as normal Raman spectroscopy. This means a narrow band coherent light source, an optical system delivering the excitation beam to the sample and the collected scattered light to the spectrometer, a notch or a steep edge filter stopping the light at the excitation wavelength to enter the spectrometer, a high-resolution spectrometer, and a sensitive detector. ${ }^{523}$ For a given SERS application the excitation wavelength can be selected based on the properties of the SERS agent or the sample. Here, LSPR wavelength and width must be considered, as well as the optical absorption and emission properties of the analyte(s) (to exploit the benefits of resonant Raman scattering (if applicable)) and avoid the overlapping of the Raman bands by fluorescence. The possibility of the damage of the sample at a given laser wavelength should be considered as well. ${ }^{523}$

Metallic NPs and colloidal systems based on them are among the most studied platforms for SERS applications. The SERS efficiency of metallic NPs, especially of $\mathrm{Au}, \mathrm{Ag}$ and $\mathrm{Cu}$, were early recognized and investigated by SERS researchers. Similarly to LSPR, the SERS characteristics of a colloidal system also depend on the NPs shape, size, and aggregation state. ${ }^{537}$ The aggregation of NPs in the colloid leads to increased SERS, however, if aggregation is performed without control, spatial, time and sample-to-sample variations might occur. ${ }^{537}$ The positive effect of the aggregation on SERS efficiency is related to the formation of hotspots - nanosized gaps between the NPs, in which the plasmonic fields of the neighbor particles are coupled, resulting in remarkable increase of the Raman intensity. ${ }^{537}$ Fabrication of SERS platform with large number of hotspots is the aim of many developments.

The investigation of the effect of the gold NPs shape on the SERS enhancement showed that nanorods have a larger enhancement than nanospheres, but nanostars perform even better, which can be attributed to the highly concentrated electromagnetic field at the tips. ${ }^{538}$

When using metal NPs for plasmonics, SERS can be combined with LSPR for molecular identification purposes. ${ }^{539,540}$ In fact, SERS and LSPR involve some common mechanism, namely the electromagnetic enhancement or the interaction of localized surface plasmons with the electromagnetic field of incident light. This interaction of the metallic NP with light leads to the creation of a local field around it which is 

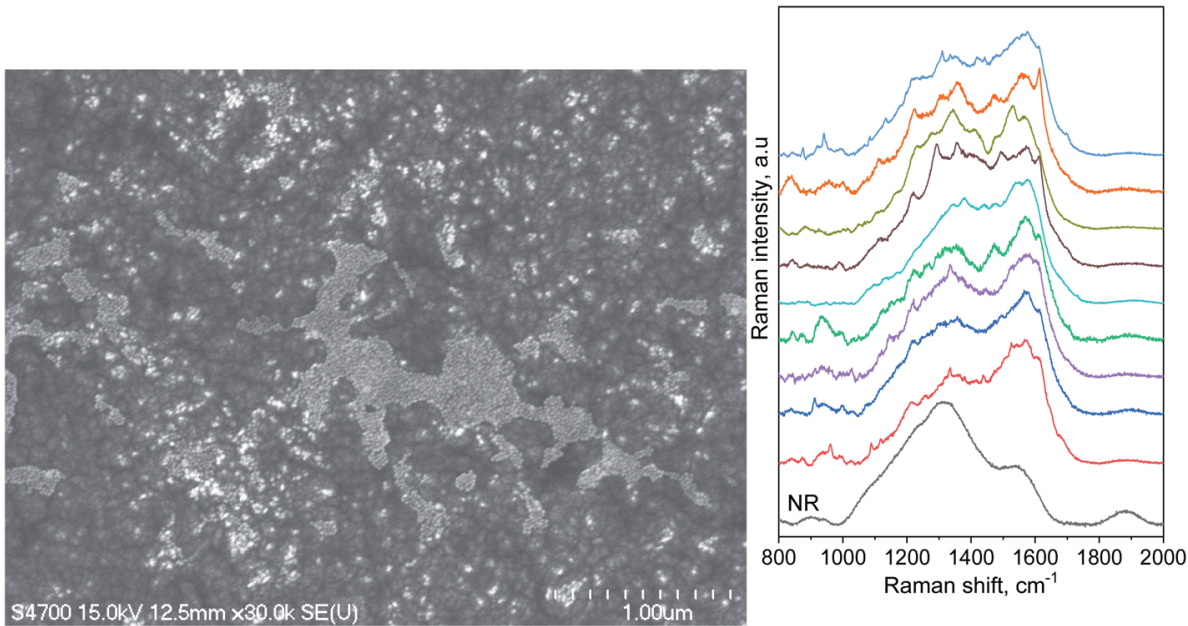

Fig. 8 (Left) Aggregated $20 \mathrm{~nm}$ sized gold NPs on the surface of a nanocrystalline diamond film having $25 \mathrm{~nm}$ average grain size and (right) normal Raman and SERS spectra recorded on this nanodiamond layer sample (based on the authors' own data).

proportional to the incident excitation. ${ }^{540}$ Once a molecule is in the vicinity of such a nanoparticle, it will be involved in a SERS process, but will also contribute to the shift of the LSPR wavelength due to its effect on the dielectric properties of the vicinity of the NP. ${ }^{540}$ This allows to perform SERS and LSPR measurements simultaneously.

A huge number of applications have been developed for SERS, including biology, medical diagnostics, trace element analysis, study of different gas, liquid and solid samples, from macroscopic sizes to and nanostructures. The local character and small SERS active region of plasmonic NPs can be used to study the surface of solid surfaces. For example, functional groups from grain boundaries of nanocrystalline diamond films can be enhanced selectively by dripping a drying gold NPs from a colloid onto the layer surface (Fig. 8). Sharp peaks appear in the SERS spectrum on top of the otherwise broad bands arising from the amorphous carbon intergrain phase.

The capabilities of SERS can be increased by combining different SERS architectures. Efficient hotspots formation has been demonstrated between two or several NPs, as well as between a flat metallic surface and the curved surface of a gold NP. ${ }^{541,542}$ An even better gain can be achieved by combining
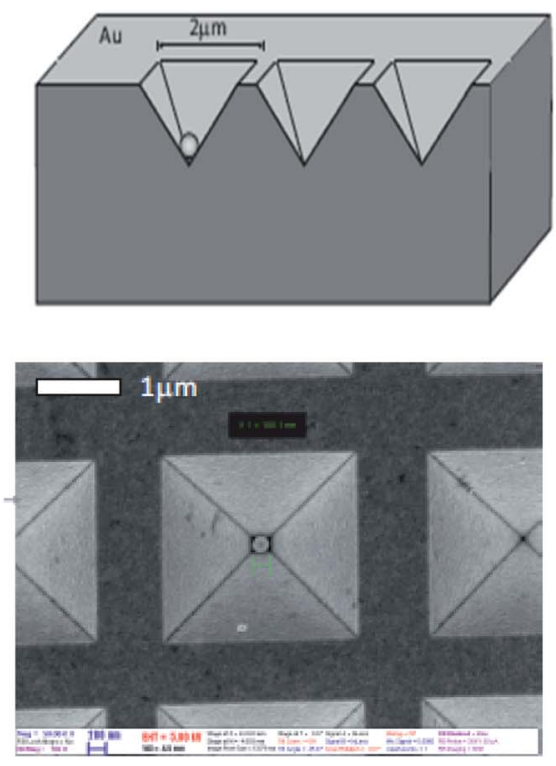

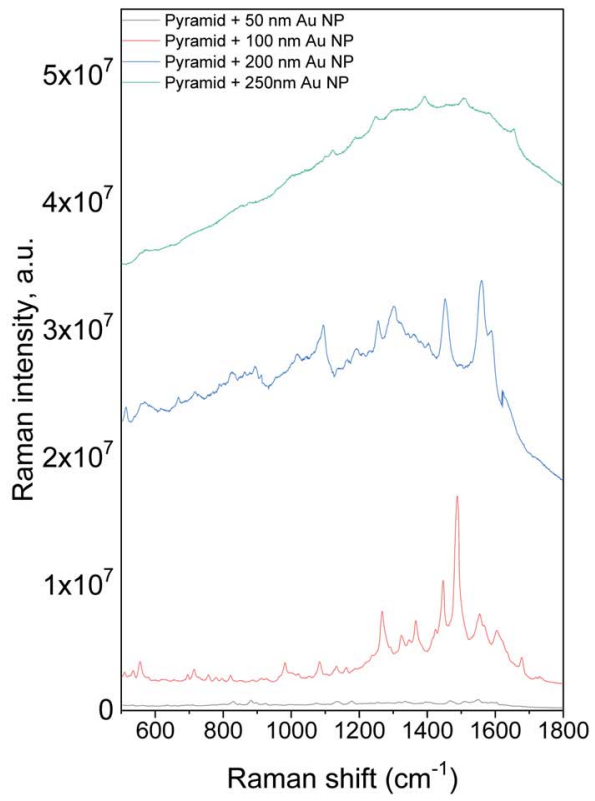

Fig. 9 (Top left) Schematic representation of the Au NP trapped in gold coated void of inverse pyramid shape, prepared in a silicon substrate (bottom left) SEM image of the fabricated structure (from the top) with a gold NP clearly visible inside the void and (right) SERS enhancement obtained with the inverse pyramid containing NPs of different size between 50 and $250 \mathrm{~nm}$ (based on the authors' own data). 
a periodic SERS array with NPs. A giant SERS and SEF enhancement has been observed in structures consisting of nanometer sized gold particles trapped in gold coated inverse pyramids fabricated in silicon by photolithographic technique $^{543}$ (Fig. 9). The enhancement was found to increase with increasing the NP size from 50 to $200 \mathrm{~nm}$, which was related to the shift of the contact point of the nanosphere and the pyramid surface towards the region with highest near-field enhancement of the pyramid.

Novel methods have been developed for cost-efficient SERS substrate fabrication for different applications. Self-assembly of metallic NPs at water-oil interface was found to be a convenient and efficient route to obtain densely packed plasmonic nanoparticles which have small interparticle distances. ${ }^{544}$ In this case, the surface charge preventing aggregation and selfassembly can be removed by using chemical modifiers on the NP surface, addition of co-solvents or charge screening by promoters, or their combination. ${ }^{544}$ After the fabrication the liquid plasmonic arrays are usually transformed into freestanding solid films. The capabilities of such 2D arrays and fabrication method were demonstrated with DNA, ${ }^{545}$ microRNA, ${ }^{546}$ multiplex analyte detection ${ }^{547}$ and others. A simple, onestep gas phase technique was used to prepare structures with tunable plasmonic enhancement utilizing spark discharge nanoparticle generation and deposition of the NPs onto glass microfiber filters. ${ }^{31}$ The Au/Ag binary NPs obtained by this method showed remarkable signal enhancement be achieved over a large surface area, and the plasmonic properties of the SERS substrate were easily tunable by changing the composition of the NPs.

4.3.3. NP-enhanced LIBS and LA-ICP-MS. Several techniques have been elaborated to increase the sensitivity of LIBS, as double-pulse excitation, spatial or magnetic confinement, spark discharge, etc. A detailed review on these methods has been published earlier. ${ }^{548}$ In the last decade, with the progress in nanotechnology, method of nanoparticle-enhanced LIBS (NELIBS) has been developed providing outstanding enhancement and relatively simple operation. In addition to the amplification another major advantage of NELIBS is the gentle ablation regime that can better preserve the sample. ${ }^{549}$ In NELIBS colloidal metallic NPs are dripped and dried on the sample surface, and the NE-LIBS is excited in those portions of the sample surface that is covered with NPs. ${ }^{550}$

The NELIBS technique has been first demonstrated on plant leaves using low-energy LIBS. ${ }^{469}$ Colloidal silver NPs were applied to the leaf surface resulting in 3-5 times enhancement of the LIBS intensity of the fresh leaf sample. NELIBS was found to be even more efficient when placing NPs on conducting (metallic) surfaces, ${ }^{551}$ while less amplification was observed in case of insulators. ${ }^{552}$ Therefore, metal samples are mostly used in NELIBS studies, but the method was also used on transparent materials, ${ }^{552,553}$ gemstones, ${ }^{553}$ solutions, ${ }^{554,555}$ fruits and vegetables, ${ }^{556}$ food $^{557,558}$ and biological tissues. ${ }^{469}$ Among these studies, the enhancement factors have shown big variations, ranging from a factor of a few to several orders of magnitude. It was shown, that the material and surface concentration of NPs, as well as the laser wavelength and irradiance all have a great impact on the signal enhancement achievable. ${ }^{12,550}$

The production of the plasma plays a crucial role in LIBS. In general, the plasma induction needs the production of free electrons, the so-called seed electrons. ${ }^{559}$ In case of solids, after vaporization and ionization of the sample this starts with multiphoton ionization, during which electrons collect the energy of multiple photons so that the total absorbed energy is enough to overcome the work function of the solid or the ionization energy of the laser vaporized atoms. ${ }^{559}$ When metal NPs are placed on the sample, the induced LSPR can enhance the electromagnetic field of the incident radiation, and this can change the way the ablation and the plasma induction occur: the laser-sample interaction will mainly be mediated by the NPs. ${ }^{559}$

According to recent studies, different mechanisms occur when metal NPs are used for LIBS in conductive and insulator samples. When NPs are deposited on a metallic surface, the LSPR enhancement of the laser field changes production of seed electrons from typical multiphoton ionization to field emission, resulting in instantaneous emission of electrons before the particles are completely deteriorated upon the effect of the laser. ${ }^{551}$ In addition, electrons accelerated upon laser irradiation provide additional electron flow that contributes to induction of an efficient breakdown and an efficient plasma excitation. ${ }^{559}$ Since field enhancement is produced on several NP hotspots simultaneously, several electron extraction points are available in the laser spot, leading to multiple ignition points of the plasma. ${ }^{559}$

When NPs are placed on an insulator sample, the LSPR enhancement is too weak to remove electrons from the bulk substrate. ${ }^{551}$ In this case, depending on the laser irradiance and excitation wavelength, two mechanism can occur. If laser is in resonance with the localized surface plasmons, the electromagnetic field enhancement due to NPs surface electron oscillations will cause strong local heating on the sample surface by the NPs. ${ }^{551}$ This can be accompanied by optical breakdown of NPs and generation of the plasma and this can extend to the sample in the close vicinity of NPs, if the irradiance is high enough. ${ }^{551}$ If the laser pulse is off-resonance with surface plasmons and the interparticle distance between NPs is small, electron transfer and charge unbalancing can occur that can initiate optical breakdown of the NPs. ${ }^{551}$

In case of semiconductors, depending on the band gap energy of the sample, NELIBS works like with metallic or dielectric samples. ${ }^{59}$ Typical examples are amorphous silicon and doped silicon. The first is a material with relatively large band gap and NELIBS does not produce any noticeable enhancement on Si emission with that, while in the additional electron energy levels introduced by doping allow to obtain 10 times enhancement of the LIBS signal. ${ }^{559}$

The issue of sample contamination after NELIBS measurement was also investigated. In ref. 552, it was found that the elements coming from NPs ablation constitute $0.004 \%$ of the total ablated material. In most cases NPs are almost completely removed from the sample after one laser shot and after three 
laser shots the sample is completely free from the NPs contamination. ${ }^{559}$

The application potential of NELIBS has been demonstrated on many different samples, ranging from metals to liquids. The highly efficient enhancement obtained on conducting surfaces and allowing the detection of trace elements to sub-ppm level put the analysis of metallic samples to the front of NELIBS research. ${ }^{552}$ The capabilities of the method were tested with titanium alloys and brass ${ }^{560}$ and other metals. ${ }^{561}$ A promising application of NELIBS is the measurement of liquid phase samples, which is based on the mechanism of NELIBS characteristic for insulators.

As it has been described earlier, the irradiation of metal NPs placed on insulator substrate with the laser pulse will initiate breakdown of the nanoparticles and plasma generation, and this plasma can include analytes being in close vicinity of those NPs. ${ }^{51}$ This can be attempted by dripping and drying the sample onto the NPs attached to a substrate. This approach was used to enhance the copper signal by delivering the sample to NPs from liquid solution. ${ }^{54}$ A sub-ppb detection limit was achieved for silver in a solution of silver nitrate. ${ }^{550}$ Modified SERS substrates consisting of Ag NPs on indium-tin-oxide glass were also used for quantitative NELIBS measurements on liquid solutions of $\mathrm{Mn}, \mathrm{Zn}$ and $\mathrm{Cr}$, and it was found that interparticle distance plays crucial role in efficient enhancement. ${ }^{\mathbf{1 2}}$

A biological sample was used as subject in the first demonstration of the NELIBS effect. A colloidal silver solution was applied to a leaf surface ${ }^{469}$ allowing the measurements of several alkaline metals with an enhancement of about a factor of 5 . This method was used to study other biological systems as well. Different types of animal fodder (artificial feed, barley, and clover) and ancient and contemporary bones were analyzed, in order to correlate their elemental composition and determine the differences between feeding of animals over time. ${ }^{558}$ Sprinkling biosynthesized silver NPs were deposited onto the bovine bone and fodder sample surface before analysis and the obtained NELIBS data were analyzed by statistical and direct evaluation. Proteins in canned tuna fish were also assessed using NELIBS technique, and the method was found to be promising for production monitoring of canned foods containing proteins. For this study biosynthesized silver NPs were used to enhance the LIBS emission intensity of fish samples. ${ }^{557}$ Another study focused on the detection of trace pesticides and heavy metal residues in fruits and vegetables. Compared to standard LIBS, NELIBS gave two orders of magnitude lower detection limit for these contaminants and allowed to perform mapping measurements on vegetable leaves. ${ }^{556}$

Laser Ablation Inductively Coupled Plasma Mass Spectrometry (LA-ICPMS) is another highly sensitive and fast analytical technique, allowing to perform elemental analysis on solid samples by ablating their surface and determining the composition of the formed particulate material via mass spectrometry. ${ }^{562}$ It requires little or no sample preparation, the analysis can be performed on conducting, non-conducting, opaque and transparent materials; and allows to perform bulk analysis, depth profiling or elemental/isotope mapping. ${ }^{\mathbf{5 6 2}}$
While the operating principle of LIBS and LA-ICPMS are different, they are common in the way of the excitation of the sample to be measured. ${ }^{562}$ The sample is illuminated with an intense laser pulse that ablates its surface. The generated plasma is detected by LIBS, while the particles are transported to the ICP torch and from there into the mass spectrometer. So, it can be expected that the NP enhancement working for LIBS should improve the performance of LA-ICPMS as well. This has been demonstrated first on conductive samples by achieving an enhancement of two orders of magnitude. ${ }^{40}$ The sample preparation was like in case of NELIBS: gold and silver NPs were dried onto the sample surface. The successful demonstration of NE-LAICPMS on conductive materials was accompanied with measurement on insulating ceramic and glass samples, however, no enhancement has been observed for the latter. ${ }^{40}$ Later it was shown that the effect works for metallic elements in both conductive and dielectric matrices. ${ }^{562}$ The NE-LAICPMS enhancement was found to be dependent on both the typology, concentration and size of the dropped nanoparticles and the composition of the sample, and different elements showed different enhancement in the same matrix, just as the same element showed different enhancement in different matrices. ${ }^{\mathbf{5 6 2}}$ Study of the effect of NPs on the aerosol formed during laser ablation showed that the highest number of particles was generated using the smallest (10 nm) particles, and, in contrast to normal LAICPMS, where the number of particles increased in proportion to the used fluence, the aerosol generation efficiency showed a maximum for NE-LAICPMS at moderate laser powers.

The origin of this behavior was attributed to two reasons. ${ }^{563}$ The first is related to the plasmonic properties of NPs, similarly to LIBS: the gaps between close NPs work as ignition points of plasma, and in this way whole plasma dynamics changed. This results in the formation of smaller particles at the expense of larger ones resulting in more efficient transport, evaporation and ionization in ICP. The second is the change of the optical properties of the surface due to the presence of NPs, increasing the energy transfer of the laser and the efficiency of the ablation of the sample, and thus leading to higher mass production. ${ }^{563}$

4.3.4. NP-enhanced laser fluorescence spectroscopy. Metalenhanced or surface-enhanced fluorescence (or SEF) on metallic NPs was first reported in the early $80 \mathrm{~s}$, as simultaneous amplification of normal and resonant Raman scattering, and fluorescence from molecules adsorbed on silver-island films. ${ }^{\mathbf{4 6 5}}$ Nowadays this field is developing together with other surface plasmon related techniques, like LSPR or SERS. SEF occurs when fluorophores are excited near a metallic surface, at a distance of a few tens of nanometers. ${ }^{564}$ Interestingly, the fluorescence emission is quenched in case the fluorophore is in contact or in close vicinity of the metallic surface, which gives a remarkable advantage for SERS over normal Raman scattering affected by strong fluorescence background in many cases. Because of the latter, there should be some definite distance with maximum SEF enhancement, however, this has not been determined yet. In general, quenching dominates at a few nanometers distance from the metal surface, followed by an exceptionally large enhancement in the $10-50 \mathrm{~nm}$ range, and then the emission turns into normal fluorescence with further increasing distance. The enhancement factor of SEF on metallic 
NPs was found to reach even a few thousands and was shown to be dependent on their material, size, and shape. ${ }^{\mathbf{4 5 4}}$

The mechanism of SEF is not precisely determined yet. The main reason is most probably the presence of different interactions between the fluorophore (analyte) and the metallic surface. SEF is supposed to be a complex phenomenon involving surface plasmons and optical near-fields, enhancing the fluorescence intensity and photostability, but also affecting its lifetime. ${ }^{565}$ The electromagnetic interactions involved in SEF can be divided into three categories: LSPR, non-radiative energy transfer, and intrinsic radiative decay. ${ }^{418}$ Some of these coupling interactions are related to the spectral overlap between surface plasmons and the emission band of the analyte, and each of them dominates SEF on different separation distances between the fluorophore and the metallic NP. ${ }^{566,567}$

Localized surface plasmon resonance influences SEF through the increase of the excitation rate, by enhancing the local electromagnetic field. This effect is similar to the electromagnetic enhancement of SERS. It is supposed that the LSPR generated on metallic NPs modifies the absorption characteristics of the fluorophores by increasing their cross-section, ${ }^{568}$ which amplifies the fluorescence intensity by coupling with the NPs. ${ }^{454}$ The geometry of the NPs plays crucial role is this interaction, since due to the field effect, SEF enhancement will be stronger at the edges and corners of NPs of complex size, ${ }^{\mathbf{5 6 9 , 5 7 0}}$ but the NP size and shape affect the LSPR conditions (maximum wavelength and spectral width) as well.

The non-radiative interaction mediates the surface plasmons' interaction with the fluorophore. ${ }^{418}$ The character of this effect determines whether quenching or enhancement of the fluorescence will occur: ${ }^{571}$ the energy transfer between the surface plasmons and the analyte will increase the fluorescence intensity only in some distance between the analyte and the metallic surface. According to recent studies, the fluorescence enhancement achieved within the distance of up to $10-15 \mathrm{~nm}$ from the metallic surface can be explained through the Forster resonance energy transfer (FRET) ${ }^{572}$ (or non-radiative dipole-dipole coupling), decaying rapidly with distance. For larger separations (10-50 nm) SEF can be explained through the role the NP characteristics play in light coupling (the so-called Purcell effect). ${ }^{564}$ It was shown that at such distances the enhancement is higher on metallic structures absorbing light rather than scattering it, as well as on NPs having concentrated and confined electromagnetic fields around sharp edges or narrow gaps. ${ }^{573}$

The role of intrinsic radiative decay is also more remarkable for the case when the analytes and the NPs are in close vicinity of each other. In this case the fluorophore is coupled to surface plasmons of NP generating new SEF decay pathways for energy transfer. The analytes are excited by the non-radiative energy transfer from NPs and transmit this energy to the far-field through radiative transfer, resulting in enhanced fluorescence intensity. ${ }^{567}$ The rate of the energy transfer can be controlled by fine-tuning the metallic structures, which, due to the enhanced rate of radiative decay, will further decrease the fluorescence lifetime of the fluorophore. ${ }^{567}$

As it can be expected, SEF is strongly affected by characteristics of the metallic NPs. The type of metal determines the useful plasmonic wavelength region, which is further narrowed by the particles' size and shape. The NPs size is also related to the SEF specific "scattering to absorption ratio" affecting the non-radiative interaction (interestingly, the latter was found to be independent of the aspect ratio for $\mathrm{SEF}^{574}$ ). In general, absorption is dominant for NPs smaller than $20 \mathrm{~nm}$, and scattering occurs for larger sizes and further increases with the size of the particles. Shape plays a remarkable role in determining the spectral properties of NPs: ${ }^{574}$ elongated and complex forms can be used to fabricate structures with dual LSPR peaks and specific hotspot regions, where the electromagnetic field enhancement by LSPR can be concentrated. ${ }^{575} \mathrm{~A}$ summary of various nanostructures and NPs with different shapes and geometries, and their experimentally determined SEF enhancement factors are provided in ref. 567. It can also be concluded from there that, in addition to shape, the enhancement of metallic NPs critically depends also on their interparticle distance, dielectric constant, and physical dimensions.

Since surface enhanced fluorescence emission is quenched if the analyte is in close vicinity of the surface of NPs, the most important and critical criterion for efficient SEF is to fabricate a layer of non-metallic material on the NP surface, referred to as a spacer. ${ }^{576,577}$ A large variety of spacers have been reported that, based on the materials used for distance modulation, can primarily be divided into three categories: inorganic (e.g., silica), organic (e.g., polymer, proteins, DNA, and others) and hybrid spacers. ${ }^{578}$ In case of metallic NPs, the spacer on the surface of the particle is obtained by fabrication of core-shell structures. These structures are defined as consisting of an inner isolated NP coated with one or several shell layers, or as inverse core-shell systems with the fluorophore encapsulated into a hollow core metallic NP.

Preparation of core-shell SEF NPs requires multiple steps. ${ }^{579}$ The first step is the fabrication of the nanoparticles; this can be done using different metals (gold, silver, copper, zinc, alloy ${ }^{580}$ ), obtaining nanoobjects of various size and shape, including rods, triangles, cubes, stars. ${ }^{581}$ In this step, the plasmonic properties of the metallic core can be engineered according to the requirements of the particular SEF application. After their fabrication, the metallic NPs should be stabilized against agglomeration by the formation of an appropriate surface layer. Here particular attention should be paid not to affect their unique optical properties. This can be achieved by using molecular layers, or even thick coatings that could serve as base layer of the spacer. ${ }^{579}$ When selecting the stabilizer, in addition to its dielectric characteristics, the compatibility with the spacer material must also be taken into account. The third, crucial step, having significant impact on the efficiency of the SEF agent is the fabrication of the spacer itself. This is followed by functionalization of the silica surface and the attachment of the fluorophore through electrostatic attraction or covalent binding. ${ }^{578}$ Various kinds of fluorophores have been assembled onto the silver-silica and gold-silica core-shell NPs, ${ }^{582,583}$ providing SEF with enhancement factors ranging from 2 to 20 .

Since the role of the spacer is to provide elevation for the analyte from the quenching zone to the SEF distance, spacers must be of uniform and precisely engineered thickness, which 
is not so easy to control on the nanoscale. ${ }^{579}$ The simplest method for this is the layer-by-layer deposition of polymer, dielectric or hybrid composite spacers. It was found that multilayered films with hybrid nanocomposites deliver much higher intensity SEF. ${ }^{\mathbf{5 9 , 5 8 4 , 5 8 5}}$ Various materials such as polyelectrolytes were used as building blocks to fabricate functional multilayered thin films for SEF, composed of sequentially stacked functional materials such as DNA probes or small nanoparticles $^{586}$ and the fluorophores positioned to the last layer. The multilayer approach can be used to obtain stimuliresponsive structures by incorporating materials being sensitive to temperature, $\mathrm{pH}$, light, etc. that can be used to control the properties of SEF agents. ${ }^{579,587}$

In colloidal NP systems the enhancement factors in suspensions were found to be up to several orders of magnitude. In the first studies silver core and silica shell particles were used in a solution sensing platform. ${ }^{583}$ These monodisperse structures were called as metal-enhanced fluorescence nanoballs, the performance of which was demonstrated for potential applications in cellular imaging and solution-based sensing by incorporating near-infrared emitting probes within the silica shell. ${ }^{583}$ Due to its chemical inertness, good optical transparency, biocompatibility, and low toxicity ${ }^{588,589}$ silica is amongst the most widely used inorganic spacer materials in colloidal systems. Another advantage of silica spacers is the possibility to obtain uniform thickness up to $90 \mathrm{~nm}$ by controlling only the silica precursor concentration during the fabrication process. ${ }^{590}$ In addition, the surface of silica could easily be modified with different functional groups, enabling simple conjugation of fluorophores and biomolecules.

Beside metallic core and dielectric shell NP configurations, silica-based hybrid nanocomposite spacer systems can be used in form of dye-silica inner beads decorated with metal NPs or surrounded by a continuous thin metal shell. ${ }^{591}$ Having a favorable cavity size, the emission properties of the fluorophore can be enhanced significantly in such structures, which can be explained by two effects: the LSPR enhancement of the exciting electromagnetic field within the cavity, and the reduction of the fluorescence lifetime due to the strong near-field interaction between the fluorophore and cavity. ${ }^{592}$ Further advantages of such nanostructures include the tunability of the LSPR wavelength by controlling the core to shell ratio, ${ }^{593}$ the standardization of surface functionalization: the same metallic shell can be used with a broad variety of fluorophores ${ }^{578}$ and the possibility to combine SEF with SERS. ${ }^{582}$ The general fabrication procedure of inverse core-shell structures consists of coating the fluorophore with silica of the required thickness, followed by surface functionalization of the outside silica surface and the preparation of the metallic shell in form of continuous film or a layer of small metallic NPs. ${ }^{578}$

SEF has numerous applications in various fields of chemical analysis and especially of biosensing and medical diagnostics. A number of review papers were published on this topic in the past few years ${ }^{454,579,594-596}$ indicating the versatility of applications and the rapid development of SEF.

\section{Conclusions}

In the present review, we attempt to provide the reader with a comprehensive overview of the progress in the field of nanoparticle-related laser and plasma spectroscopy research. We believe that the results disseminated recently in the literature properly illustrate the symbiotic connection between materials science and analytical laser/plasma spectroscopy. This connection is not one-directional, but there are multiple ways through which these research areas can benefit from each other and thereby enjoy a mutually supportive relationship. As we have shown, modern laser and plasma spectroscopy technologies offer versatile and effective methods for nanoparticle characterization and monitoring nanoparticle synthesis, thereby also contributing to the understanding of nanoparticle formation mechanisms. At the same time, materials science can boost the performance of laser and plasma spectroscopy by providing suitable and tunable nanoparticles useful for plasmonic analytical signal enhancement. Based on the rate of progress in the past decades, it can be expected that this relationship will flourish in the future as well. As an example, interesting recent fundamental works dealing with the directional emission of electrons and ions from isolated nanoparticles induced by femto- and attosecond laser impulses can be mentioned. ${ }^{597,598}$ The combination of nm-scale sampling on sub-fs time scales could open new analytical possibilities for the monitoring of near-field induced reaction yields on the surface of nanoparticles ("reaction nanoscopy") ${ }^{599}$ or for the differentiation of single particles and their dimers based on their plasmonic characteristics, ${ }^{600}$ to name just a couple of options.

\section{Conflicts of interest}

There are no conflicts of interest to declare.

\section{Acknowledgements}

The funding received from the National Research, Development and Innovation Office of Hungary through projects K 129063, GINOP-2.3.2-15-2016-00036, EFOP-3.6.2-16-2017-00005 and VEKOP-2.3.2-16-2016-00011, and from the Ministry for Innovation and Technology of Hungary through the NKFIH-1279-2/ 2020 TKP 2020 program is acknowledged. One of the authors (A. Kohut) is also grateful for the support received from the UNKP-20-4 - New National Excellence Program of the Ministry for Innovation and Technology from the source of the National Research, Development and Innovation Fund. The open access publication of this work has been supported by the University of Szeged Open Access Fund, under Grant No. 5391.

\section{References}

1 A. Schmidt-Ott, Spark Ablation: Building Blocks for Nanotechnology, Jenny Stanford Publishing, Boca Raton, 1st edn, 2020.

2 R. Vajtai, Springer Handbook of Nanomaterials, Springer, Berlin, Heidelberg, 1st edn, 2013. 
3 A. V. Nikam, B. L. V. Prasad and A. A. Kulkarni, CrystEngComm, 2018, 20, 5091-5107.

4 L. Xu, X. Qi, X. Li, Y. Bai and H. Liu, Talanta, 2016, 146, 714726.

5 R. Lucena, B. M. Simonet, S. Cárdenas and M. Valcárcel, J. Chromatogr. A, 2010, 1218, 620-637.

6 J. Lei and H. Ju, Chem. Soc. Rev., 2012, 41, 2122-2134.

7 P. C. Ray, Chem. Rev., 2010, 110, 5332-5365.

8 M. E. Stewart, C. R. Anderton, L. B. Thompson, J. Maria, S. K. Gray, J. A. Rogers and R. G. Nuzzo, Chem. Rev., 2008, 108, 494-521.

9 X. Jiang, K. Huang, D. Deng, H. Xia, X. Hou and Ch. Zheng, TrAC, Trends Anal. Chem., 2012, 39, 38-59.

10 J. Langer, D. J. de Aberasturi, J. Aizpurua, R. A. AlvarezPuebla, B. Auguie, et al., ACS Nano, 2020, 14, 28-117.

11 A. Kohut, A. Kéri, V. Horváth, J. Kopniczky, T. Ajtai, B. Hopp, G. Galbács and Z. Geretovszky, Appl. Surf. Sci., 2020, 531, 147268.

12 D. J. Palásti, P. Albrycht, P. Janovszky, K. Paszkowska, Z. Geretovszky and G. Galbács, Spectrochim. Acta, Part B, 2020, 166, 105793.

13 S. Mourdikoudis, R. M. Pallares and N. T. K. Thanh, Nanoscale, 2018, 10, 12871-12934.

14 C. Fornaguera and C. Solans, Int. J. Polym. Sci., 2018, 2018, 6387826.

15 A. Kohut, L. Ludvigsson, B. O. Meuller, K. Deppert, M. E. Messing, G. Galbács and Z. Geretovszky, Nanotechnology, 2017, 28, 475603.

16 B. Ferreira da Silva, S. Pérez, P. Gardinalli, R. K. Singhal, A. A. Mozeto and D. Barcelo, TrAC, Trends Anal. Chem., 2011, 30, 528-540.

17 C. M. Hussain, Handbook of Nanomaterials in Analytical Chemistry, Elsevier, Amsterdam, 1st edn, 2020.

18 M. Valcárcel and A. I. López-Lorente, Gold Nanoparticles in Analytical Chemistry, Comprehensive Analytical Chemistry, Elsevier, Amsterdam, 2014, vol. 66.

19 S. Shtykov, Nanoobjects and Nanotechnologies in Analytical Chemistry, de Gruyter, Berlin, 2018.

20 M. Sajid and J. Płotka-Wasylka, Microchem. J., 2020, 154, 104623.

21 M. Valcárcel, S. Cardenas and B. M. Simonet, Anal. Chem., 2007, 79, 4788-4797.

22 F. Adams and C. Barbante, Chemical Imaging Analysis, Comprehensive Analytical Chemistry, Elsevier, Amsterdam, 2015, vol. 69.

23 A. S. de Dios and M. E. Díaz-García, Anal. Chim. Acta, 2010, 666, 1-22.

24 O. Axner and G. Galbács, in Encyclopedia of Analytical Chemistry, ed. R. A. Meyers, John Wiley \& Sons, Hoboken, New Jersey, 2012, Laser spectrometric techniques in analytical atomic spectrometry, pp. 1-113.

25 J. Pareja, S. López, D. Jaramillo, D. W. Hahn and A. Molina, Appl. Opt., 2013, 52, 2470-2477.

26 O. A. Nassef and H. E. Elsayed-Ali, Spectrochim. Acta, Part B, 2005, 60, 1564-1572.

27 Q. Lin, G. Niu, Q. Wang, Q. Yu and Y. Duan, Appl. Spectrosc. Rev., 2013, 48, 487-508.
28 M. Bonta, J. J. Gonzalez, C. Derrick Quarles, R. E. Russo, B. Hegedüs and A. Limbeck, J. Anal. At. Spectrom., 2016, 31, 252-258.

29 M. Tarik, G. Lotito, J. A. Whitby, J. Koch, K. Fuhrer, M. Gonin, J. Michler, J.-L. Bolli and D. Günther, Spectrochim. Acta, Part B, 2009, 64, 262-270.

30 I. B. Gornushkin, J. E. Kim, B. W. Smith, S. A. Baker and J. D. Winefordner, Appl. Spectrosc., 1997, 51, 1055-1059.

31 A. Kohut, A. Kéri, V. Horváth, J. Kopniczky, T. Ajtai, B. Hopp, G. Galbács and Z. Geretovszky, Appl. Surf. Sci., 2020, 531, 147268.

32 D. Zhang, B. Gökce and S. Barcikowski, Chem. Rev., 2017, 117, 3990-4103.

33 T. Amodeo, C. Dutouquet, F. Tenegal, B. Guizard, H. Maskrot, O. Le Bihan and E. Fréjafon, Spectrochim. Acta, Part B, 2008, 63, 1183-1190.

34 P. Purohit, F. J. Fortes and J. J. Laserna, Angew. Chem., Int. Ed., 2017, 56, 1-6.

35 P. Purohit, F. J. Fortes and J. J. Laserna, Anal. Chem., 2019, 91, 7444-7449.

36 D. Mozhayeva and C. Engelhard, J. Anal. At. Spectrom., 2020, 35, 1740-1783.

37 M. D. Montano, J. W. Olesik, A. G. Barber, K. Challis and J. F. Ranville, Anal. Bioanal. Chem., 2016, 408, 5053-5074.

38 R. Xu, Particuology, 2015, 18, 11-21.

39 M. Pintér, T. Ajtai, G. Kiss-Albert, D. Kiss, N. Utry, P. Janovszky, D. Palásti, T. Smausz, A. Kohut, B. Hopp, G. Galbács, Á. Kukovecz, Z. Kónya, G. Szabó and Z. Bozóki, Atmos. Environ., 2018, 178, 118-128.

40 M. Holá, Z. Salajková, A. Hrdlička, P. Pořízka, K. Novotný, L. Čelko, P. Šperka, D. J. Novotný, P. Modlitbová, V. Kanický and J. Kaiser, Anal. Chem., 2018, 90, 1182011826.

41 M. Dell'Aglio, R. Alrifai and A. De Giacomo, Spectrochim. Acta, Part B, 2018, 148, 105-112.

42 Z. Abdullaeva, Synthesis of Nanoparticles and Nanomaterials: Biological Approaches, Springer Nature Switzerland, Cham, 1st edn, 2017.

43 A. S. H. Makhlouf and A. Barhoum, Fundamentals of Nanoparticles, Elsevier, Amsterdam, 1st edn, 2018.

44 M. Naito, T. Yokoyama, K. Hosokawa and K. Nogi, Nanoparticle Technology Handbook, Elsevier, Amsterdam, 3rd edn, 2018.

45 N. G. Semaltianos, Crit. Rev. Solid State Mater. Sci., 2010, 35, 105-124.

46 T. V. Pfeiffer, J. Feng and A. Schmidt-Ott, Adv. Powder Technol., 2014, 25, 56-70.

47 P. Purohit, F. J. Fortes and J. J. Laserna, Anal. Chem., 2021, 93, 2635-2643.

48 A. Scheeline, J. A. Norris, J. C. Travis, J. R. Devoe and J. P. Walters, Spectrochim. Acta, Part B, 1981, 36, 373-383.

49 A. Scheeline, Mikrochim. Acta, 1990, 100, 247-285.

50 P. W. J. M. Boumans, in Analytical Emission Spectroscopy, ed. E. L. Grove, Marcel Dekker, New York, 1972.

51 C. A. Bye and A. Scheeline, Appl. Spectrosc., 1993, 47, 20222030. 
52 C. Schulz, B. F. Kock, M. Hofmann, H. Michelsen, S. Will, B. Bougie, R. Suntz and G. Smallwood, Appl. Phys. B: Lasers Opt., 2006, 83, 333-354.

53 D. Mukherjee, A. Rai and M. R. Zachariah, Aerosol Sci., 2006, 37, 677-695.

54 B. K. McMillin, P. Biswas and M. R. Zachariah, J. Mater. Res., 1996, 11, 1552-1561.

55 X. Liu, M. E. Smith and S. D. Tse, Appl. Phys. B: Lasers Opt., 2010, 100, 643-653.

56 A. O'Keefe and D. A. G. Deacon, Rev. Sci. Instrum., 1988, 59, 2544-2551.

57 I. H. Hutchinson, Principles of Plasma Diagnostics, Cambridge University Press, Cambridge, 2nd edn, 2002.

58 H. R. Griem, Plasma Spectroscopy, McGraw-Hill, New York, 1st edn, 1964.

59 H. R. Griem, Principles of Plasma Spectroscopy, Cambridge University Press, Cambridge, 1997.

60 H.-J. Kunze, Introduction to Plasma Spectroscopy, Springer, Berlin, Heidelberg, 1st edn, 2009.

61 A. R. Hanna and E. R. Fisher, J. Vac. Sci. Technol., A, 2020, 38, 020806.

62 J. A. Aguilera and C. Aragón, Spectrochim. Acta, Part B, 2008, 63, 784-792.

63 C. A. Bye and A. Scheeline, Spectrochim. Acta, Part B, 1993, 48, 1593-1605.

64 A. Scheeline, G. J. Kamla and M. J. Zoellner, Spectrochim. Acta, Part B, 1984, 39, 677-691.

65 W. L. Wiese, Spectrochim. Acta, Part B, 1991, 46, 831-841.

66 C. Yubero, M. D. Calzada and M. C. Garcia, J. Phys. Soc. Jpn., 2005, 74, 2249-2254.

67 N. Konjević and W. L. Wiese, J. Phys. Chem. Ref. Data, 1990, 19, 1307-1385.

68 J. M. Palomares, S. Hübner, E. A. D. Carbone, N. De Vries, E. M. Van Veldhuizen, A. Sola, A. Gamero and J. J. A. M. Van Der Mullen, Spectrochim. Acta, Part B, 2012, 73, 39-47.

69 C. Aragón and J. A. Aguilera, Spectrochim. Acta, Part B, 2008, 63, 893-916.

70 W. I. Wiese, Natl. Stand. Ref. Data Ser., 1969, 22, 306.

71 J. A. Aparicio, M. A. Gigosos, V. R. Gonzalez, C. Perez, M. I. de la Rosa and S. Mar, J. Phys. B: At., Mol. Opt. Phys., 1998, 31, 1029-1048.

72 W. L. Wiese and J. R. Fuhr, J. Phys. Chem. Ref. Data, 2007, 36, 1287-1345.

73 G. V. Vogman and U. Shumlak, Rev. Sci. Instrum., 2011, 82, 103504.

74 R. M. van der Horst, T. Verreycken, E. M. van Veldhuizen and P. J. Bruggeman, J. Phys. D: Appl. Phys., 2012, 45, 345201.

75 C. Yubero, M. S. Dimitrijević, M. C. García and M. D. Calzada, Spectrochim. Acta, Part B, 2007, 62, 169-176.

76 D. Xiao, C. Cheng, J. Shen, Y. Lan, H. Xie, X. Shu, Y. Meng, J. Li and P. K. Chu, Phys. Plasmas, 2014, 21, 053510.

77 H. Onishi, F. Yamazaki, Y. Hakozaki, M. Takemura, A. Nezu and H. Akatsuka, Jpn. J. Appl. Phys., 2021, 60, 026002.

78 A. A. Fridman and L. A. Kennedy, Plasma Physics and Engineering, CRC Press, Boca Raton, Florida, 2nd edn, 2011.
79 K. Yambe and S. Satou, Phys. Plasmas, 2016, 23, 023509.

80 T. Atwee, L. Aschke and H. J. Kunze, J. Phys. D: Appl. Phys., 2000, 33, 2263-2267.

81 F. Rezaei, Appl. Opt., 2020, 59, 3002.

82 H. Zhang, Y. Wu, H. Sun, F. Yang, M. Rong, F. Jiang, C. Wang and W. Huang, Plasma Chem. Plasma Process., 2019, 39, 1429-1447.

83 H. R. Griem, Phys. Rev., 1963, 131, 1170-1176.

84 R. W. P. McWhirter, in Plasma Diagnostic Techniques, ed. R. H. Huddlestone and S. L. Leonard, Academic Press, New York, 1st edn, 1965, ch. 5, pp. 201-264.

85 A. Kohut, G. Galbács, Z. Márton and Z. Geretovszky, Plasma Sources Sci. Technol., 2017, 26, 045001.

86 G. Cristoforetti, A. De Giacomo, M. Dell'Aglio, S. Legnaioli, E. Tognoni, V. Palleschi and N. Omenetto, Spectrochim. Acta, Part B, 2010, 65, 86-95.

87 V. K. Unnikrishnan, K. Alti, V. B. Kartha, C. Santhosh, G. P. Gupta and B. M. Suri, Pramana, 2010, 74, 983-993.

88 H. R. Griem, Phys. Rev., 1962, 128, 997-1003.

89 J. A. Aguilera and C. Aragón, Spectrochim. Acta, Part B, 2007, 62, 378-385.

90 L. Sun and H. Yu, Talanta, 2009, 79, 388-395.

91 B. Praher, V. Palleschi, R. Viskup, J. Heitz and J. D. Pedarnig, Spectrochim. Acta, Part B, 2010, 65, 671-679.

92 A. Safi, S. H. Tavassoli, G. Cristoforetti, S. Legnaioli, V. Palleschi, F. Rezaei and E. Tognoni, J. Adv. Res., 2019, 18, 1-7.

93 G. J. Bastiaans and R. A. Mangold, Spectrochim. Acta, Part B, 1985, 40, 885-892.

94 P. Bruggeman, N. Sadeghi, D. C. Schram and V. Linss, Plasma Sources Sci. Technol., 2014, 23, 023001.

95 S. E. Pratsinis, Prog. Energy Combust. Sci., 1998, 24, 197-219.

96 H. K. Kammler, L. Mädler and S. E. Pratsinis, Chem. Eng. Technol., 2001, 24, 583-596.

97 F. Schneider, PhD thesis, University of Duisburg-Essen, 2020.

98 J. Wei, Y. Ren, Y. Zhang, B. Shi and S. Li, J. Aerosol Sci., 2019, 133, 72-82.

99 F. Meierhofer, L. Mädler and U. Fritsching, AIChE J., 2020, 66, 66.

100 T. Dreier and C. Schulz, Powder Technol., 2016, 287, 226238.

101 C. Schulz, T. Dreier, M. Fikri and H. Wiggers, Proc. Combust. Inst., 2019, 37, 83-108.

102 I. Rahinov, J. Sellmann, M. R. Lalanne, M. Nanjaiah, T. Dreier, S. Cheskis and I. Wlokas, Energy Fuels, 2021, 35, 137-160.

103 C. Liu, Z. Cao, F. Li, Y. Lin and L. Xu, Meas. Sci. Technol., 2017, 28, 054002.

104 R. S. M. Chrystie, O. M. Feroughi, T. Dreier and C. Schulz, Appl. Phys. B: Lasers Opt., 2017, 123, 104.

105 R. S. M. Chrystie, F. L. Ebertz, T. Dreier and C. Schulz, Appl. Phys. B: Lasers Opt., 2019, 125, 29.

106 A. El Moussawi, T. Endres, S. Peukert, S. Zabeti, T. Dreier, M. Fikri and C. Schulz, Combust. Flame, 2021, 224, 260-272. 
107 R. Mansmann, T. Terheiden, P. Schmidt, J. Menser, T. Dreier, T. Endres and C. Schulz, Appl. Phys. B: Lasers Opt., 2018, 124, 69.

108 R. Mansmann, T. A. Sipkens, J. Menser, K. J. Daun, T. Dreier and C. Schulz, Appl. Phys. B: Lasers Opt., 2019, 125, 126.

109 G. Galbács, Anal. Bioanal. Chem., 2015, 407, 7537-7562.

110 Y. Ren, Y. Zhang and S. Li, Proc. Combust. Inst., 2019, 37, 1373-1381.

111 P. R. Willmott and J. R. Huber, Rev. Mod. Phys., 2000, 72, 315-328.

112 D. A. Cremers and L. J. Radziemski, Handbook of Laser-Induced Breakdown Spectroscopy, John Wiley \& Sons, Hoboken, New Jersey, 2nd edn, 2013.

113 J. Singh and S. Thakur, Laser-Induced Breakdown Spectroscopy, Elsevier, Amsterdam, 2nd edn, 2020.

114 A. De Giacomo, M. Dell'Aglio, A. Santagata, R. Gaudiuso, O. De Pascale, P. Wagener, G. C. Messina, G. Compagnini and S. Barcikowski, Phys. Chem. Chem. Phys., 2013, 15, 3083-3092.

115 Q. Xiao, Z. Yao, J. Liu, R. Hai, H. Y. Oderji and H. Ding, Thin Solid Films, 2011, 519, 7116-7119.

116 S. Dadras, M. J. Torkamany and P. Jafarkhani, J. Nanosci. Nanotechnol., 2012, 12, 3115-3122.

117 P. Nancy, J. James, S. Valluvadasan, R. A. V. Kumar and N. Kalarikkal, Nano-Struct. Nano-Objects, 2018, 16, 337-346.

118 V. Narayanan and R. K. Thareja, Appl. Surf. Sci., 2004, 222, 382-393.

119 B. Kumar and R. K. Thareja, Phys. Plasmas, 2013, 20, 053503.

120 M. Oujja, J. J. Camacho, M. Sanz, M. Castillejo and R. de Nalda, J. Quant. Spectrosc. Radiat. Transfer, 2020, 256, 107308.

121 M. Sanz, M. López-Arias, E. Rebollar, R. De Nalda and M. Castillejo, J. Nanopart. Res., 2011, 13, 6621-6631.

122 D. Grojo, J. Hermann and A. Perrone, J. Appl. Phys., 2005, 97, 063306.

123 A. Guarnaccio, G. P. Parisi, D. Mollica, A. De Bonis, R. Teghil and A. Santagata, Spectrochim. Acta, Part B, 2014, 101, 261-268.

124 M. Dell'Aglio, R. Gaudiuso, R. Elrashedy, O. De Pascale, G. Palazzo and A. De Giacomo, Phys. Chem. Chem. Phys., 2013, 15, 20868-20875.

125 J. Lam, D. Amans, F. Chaput, M. Diouf, G. Ledoux, N. Mary, K. Masenelli-Varlot, V. Motto-Ros and C. Dujardin, Phys. Chem. Chem. Phys., 2014, 16, 963-973.

126 A. Matsumoto, A. Tamura, T. Honda, T. Hirota, K. Kobayashi, S. Katakura, N. Nishi, K. I. Amano, K. Fukami and T. Sakka, J. Phys. Chem. C, 2015, 119, 26506-26511.

127 D. Riabinina, E. Irissou, B. Le Drogoff, M. Chaker and D. Guay, J. Appl. Phys., 2010, 108, 034322.

128 T. Kato, S. Stauss, S. Kato, K. Urabe, M. Baba, T. Suemoto and K. Terashima, Appl. Phys. Lett., 2012, 101, 2010-2015.

129 S. Palanco, S. Marino, M. Gabás, S. Bijani, L. Ayala and J. R. Ramos-Barrado, Opt. Express, 2014, 22, 3991.
130 N. Smijesh, K. Chandrasekharan, J. C. Joshi and R. Philip, J. Appl. Phys., 2014, 116, 1-7.

131 T. Donnelly and J. G. Lunney, Appl. Surf. Sci., 2013, 282, 133-137.

132 N. Cimpoesu, S. Gurlui, G. Bulai, R. Cimpoesu, V.-P. Paun, S. A. Irimiciuc and M. Agop, Symmetry, 2020, 12, 109.

133 E. J. Kautz, J. Yeak, B. E. Bernacki, M. C. Phillips and S. S. Harilal, Phys. Chem. Chem. Phys., 2020, 22, 8304-8314.

134 C. M. Maguire, M. Rösslein, P. Wick and A. Prina-Mello, Sci. Technol. Adv. Mater., 2018, 19, 732-745.

135 S. Wei and K. I. Saitow, Rev. Sci. Instrum., 2012, 83, 073110. 136 D. Werner, A. Furube, T. Okamoto and S. Hashimoto, J. Phys. Chem. C, 2011, 115, 8503-8512.

137 M. Oujja, J. G. Izquierdo, L. Bañares, R. De Nalda and M. Castillejo, Phys. Chem. Chem. Phys., 2018, 20, 1695616965.

138 M. A. Valverde-Alva, T. García-Fernández, M. VillagránMuniz, C. Sánchez-Aké, R. Castañeda-Guzmán, E. Esparza-Alegría, C. F. Sánchez-Valdés, J. L. S. Llamazares and C. E. M. Herrera, Appl. Surf. Sci., 2015, 355, 341-349.

139 Y. P. Raizer, Gas Discharge Physics, Springer-Verlag, Berlin, Heidelberg, 1st edn, 1991.

140 K. Ishikawa and M. Hori, in Plasma Medical Science, ed. S. Toyokuni, F. Kikkawa, Y. Ikehara and M. Hori, Academic Press, Cambridge, Massachusetts, 1st edn, 2018, Physical and chemical basis of nonthermal plasma, pp. 5-107.

141 N. Jidenko and J. P. Borra, J. Aerosol Sci., 2004, 35, 29-40.

142 V. I. Arkhipenko, A. A. Kirillov, Y. A. Safronau, L. V. Simonchik and S. M. Zgirouski, Eur. Phys. J. D, 2012, 66, 252.

143 J. P. Borra, N. Jidenko and E. Bourgeois, Eur. Phys. J.: Appl. Phys., 2009, 47, 22804.

144 M. Stein and F. E. Kruis, Adv. Powder Technol., 2018, 29, 3138-3144.

145 BUONAPART-E, https:/cordis.europa.eu/project/id/ 280765/reporting.

146 A. A. Efimov, A. A. Lizunova, I. A. Volkov, D. A. Mylnikov, P. V. Arsenov and V. V. Ivanov, J. Phys.: Conf. Ser., 2016, 741, 012035.

147 E. M. Bazelian and Y. P. Raizer, Spark Discharge, CRC Press, Boca Raton, 1st edn, 1997.

148 B. O. Meuller, M. E. Messing, D. L. J. Engberg, A. M. Jansson, L. I. M. Johansson, S. M. Norlén, N. Tureson and K. Deppert, Aerosol Sci. Technol., 2012, 46, 1256-1270.

149 N. S. Tabrizi, M. Ullmann, V. A. Vons, U. Lafont and A. Schmidt-Ott, J. Nanopart. Res., 2009, 11, 315-332.

150 J. Feng, L. Huang, L. Ludvigsson, M. E. Messing, A. Maisser, G. Biskos and A. Schmidt-Ott, J. Phys. Chem. C, 2016, 120, 621-630.

151 G. Biskos, V. Vons, C. U. Yurteri and A. Schmidt-Ott, KONA Powder Part. J., 2008, 26, 13-35.

152 P. Intra and N. Tippayawong, Songklanakarin J. Sci. Technol., 2008, 30, 243-256.

153 P. T. Nilsson, A. C. Eriksson, L. Ludvigsson, M. E. Messing, E. Z. Nordin, A. Gudmundsson, B. O. Meuller, K. Deppert, 
E. C. Fortner, T. B. Onasch and J. H. Pagels, Nano Res., 2015, 8, 3780-3795.

154 C. R. Svensson, L. Ludvigsson, B. O. Meuller, M. L. Eggersdorfer, K. Deppert, M. Bohgard, J. H. Pagels, M. E. Messing and J. Rissler, J. Aerosol Sci., 2015, 87, 38-52. 155 A. Scheeline and D. M. Coleman, Anal. Chem., 1987, 59, 1185A-1196A.

156 J. P. Walters, Appl. Spectrosc., 1969, 23, 317-331.

157 M. A. Biondi, Appl. Sci. Res., Sect. B, 1956, 5, 157-166.

158 E. A. McLean and S. A. Ramsden, Phys. Rev., 1965, 140, A1122.

159 L. Blitzer and W. M. Cady, J. Opt. Soc. Am., 1951, 41, 440445.

160 S. Schwyn, E. Garwin and A. Schmidt-Ott, J. Aerosol Sci., 1988, 19, 639-642.

161 E. Hontañón, J. M. Palomares, M. Stein, X. Guo, R. Engeln, H. Nirschl and F. E. Kruis, J. Nanopart. Res., 2013, 15, 1957.

162 J. P. Walters and H. V. Malmstadt, Anal. Chem., 1965, 37, 1484-1503.

163 Y. Toriyabe, S. Watanabe, S. Yatsu, T. Shibayama and T. Mizuno, Appl. Phys. Lett., 2007, 91, 041501.

164 V. S. Burakov, N. A. Savastenko, N. V. Tarasenko and E. A. Nevar, J. Appl. Spectrosc., 2008, 75, 114-124.

165 N. A. Sirotkin, A. V. Khlyustova, V. A. Titov, A. S. Krayev, D. I. Nikitin, O. A. Dmitrieva and A. V. Agafonov, Plasma Chem. Plasma Process., 2020, 40, 571-587.

166 T. A. Tmenova, A. N. Veklich, V. F. Boretskij, Y. Cressault, F. Valensi, K. G. Lopatko and Y. G. Aftandilyants, Probl. At. Sci. Technol., Ser.: Plasma Phys., 2017, 107, 132-135.

167 A. Veklich, A. Lebid, T. Tmenova, V. Boretskij, Y. Cressault, F. Valensi, K. Lopatko and Y. Aftandilyants, in 22nd Symposium on Physics of Switching Arc, Brno University of Technology, Brno, 2017, pp. 28-31.

168 M. Stein, D. Kiesler and F. E. Kruis, J. Nanopart. Res., 2013, 15, 1400.

169 I. Banerjee, N. K. Joshi, S. N. Sahasrabudhe, S. Karmakar, N. V. Kulkarni, S. Ghorui, A. K. Tak, S. P. S. S. Murthy, S. V. Bhoraskar and A. K. Das, IEEE Trans. Plasma Sci., 2006, 34, 2611-2617.

170 I. Banerjee, N. K. Joshi, S. N. Sahasrabudhe, N. V. Kulkarni, S. Karmakar, R. Pasricha, S. Ghorui, A. K. Tak, S. P. S. S. Murthy, S. V. Bhoraskar and A. K. Das, IEEE Trans. Plasma Sci., 2006, 34, 1175-1182.

171 D. Delaportas, P. Svarnas, I. Alexandrou, A. Siokou, K. Black and J. W. Bradley, J. Phys. D: Appl. Phys., 2009, 42, 245204.

172 B. Bachmann, R. Kozakov, G. Gött, K. Ekkert, J.-P. Bachmann, J.-L. Marques, H. Schöpp, D. Uhrlandt and J. Schein, J. Phys. D: Appl. Phys., 2013, 46, 125203.

173 B. Santra, M. N. Shneider and R. Car, Sci. Rep., 2017, 7, 40230.

174 A. Gerakis, Y. W. Yeh, M. N. Shneider, J. M. Mitrani, B. C. Stratton and Y. Raitses, Phys. Rev. Appl., 2018, 9, 014031.

175 S. Yatom, J. Bak, A. Khrabryi and Y. Raitses, Carbon, 2017, 117, 154-162.

176 J.-P. Borra, J. Phys. D: Appl. Phys., 2006, 39, 19-54.
177 N. Jidenko, E. Bourgeois and J. P. Borra, J. Phys. D: Appl. Phys., 2010, 43, 295203.

178 E. Hontañón, J. M. Palomares, X. Guo, R. Engeln, H. Nirschl and F. E. Kruis, J. Phys. D: Appl. Phys., 2014, 47, 415201.

179 L. Jiang, Q. Li, D. Zhu, M. Attoui, Z. Deng, J. Tang and J. Jiang, Aerosol Sci. Technol., 2017, 51, 206-213.

180 Specair, http://www.specair-radiation.net/index.php, accessed 12 April 2021.

181 F. Kokai, I. Nozaki, T. Okada, A. Koshio and T. Kuzumaki, Carbon, 2011, 49, 1173-1181.

182 M. Cau, N. Dorval, B. Attal-Trétout, J. L. Cochon, A. FoutelRichard, A. Loiseau, V. Krüger, M. Tsurikov and C. D. Scott, Phys. Rev. B: Condens. Matter Mater. Phys., 2010, 81, 165416.

183 J. Picard, J. B. Sirven and O. Sublemontier, MRS Adv., 2017, 2, 1487-1491.

184 K. Ostrikov, Rev. Mod. Phys., 2005, 77, 489-511.

185 K. Ostrikov and A. B. Murphy, J. Phys. D: Appl. Phys., 2007, 40, 2223-2241.

186 M. Hundt, P. Sadler, I. Levchenko, M. Wolter, H. Kersten and K. Ostrikov, J. Appl. Phys., 2011, 109, 123305.

187 A. Mohanta, B. Lanfant, M. Asfaha and M. Leparoux, in Journal of Physics: Conference Series, Institute of Physics Publishing, Munchen, 2017, vol. 825, pp. 12010-12017.

188 A. Mohanta, B. Lanfant and M. Leparoux, Plasma Chem. Plasma Process., 2019, 39, 1161-1179.

189 L. Mangolini, J. Phys. D: Appl. Phys., 2017, 50, 373003.

190 R. A. Khoury, J. C. Ranasinghe, A. S. Dikkumbura, P. Hamal, R. R. Kumal, T. E. Karam, H. T. Smith and L. H. Haber, J. Phys. Chem. C, 2018, 122, 24400-24406.

191 J. C. Ranasinghe, A. S. Dikkumbura, P. Hamal, M. Chen, R. A. Khoury, H. T. Smith, K. Lopata and L. H. Haber, J. Chem. Phys., 2019, 151, 224701.

192 G. Chen, I. Roy, C. Yang and P. N. Prasad, Chem. Rev., 2016, 116, 2826-2885.

193 J. Shi, P. W. Kantoff, R. Wooster and O. C. Farokhzad, Nat. Rev. Cancer, 2017, 17, 20-37.

194 P. S. Fedotov, N. G. Vanifatova, V. M. Shkinev and B. Y. Spivakov, Anal. Bioanal. Chem., 2011, 400, 1787-1804.

195 Z. Gajdosechova and Z. Mester, Anal. Bioanal. Chem., 2019, 411, 4277-4292.

196 M. Hassellöv, J. W. Readman, J. F. Ranville and K. Tiede, Ecotoxicology, 2008, 17, 344-361.

197 F. Laborda, E. Bolea, G. Cepriá, M. T. Gómez, M. S. Jiménez, J. Pérez-Arantegui and J. R. Castillo, Anal. Chim. Acta, 2016, 904, 10-32.

198 M. M. Modena, B. Rühle, T. P. Burg and S. Wuttke, Adv. Mater., 2019, 31, 1901556.

199 R. Tantra, J. C. Jaman and K. N. Robinson, in Nanomaterial Characterization: An Introduction, ed. R. Tantra, John Wiley \& Sons, Inc., Hoboken, New Jersey, 1st edn, 2016, Introduction, pp. 1-24.

200 C. F. Quate, in Scanning Tunneling Microscopy and Related Methods, ed. R. J. Behm, N. García and H. Rohrer, Springer, Dordrecht, 1st edn, 1990, Surface modification with the STM and the AFM, pp. 281-297. 
201 B. Voigtländer, in Scanning Probe Microscopy, ed. B. Voigtländer, Springer Verlag, Berlin, Heidelberg, 1st edn, 2015, Building nanostructures atom by atom, pp. 349-357. 202 D. R. Baer, J. Vac. Sci. Technol., A, 2020, 38, 031201.

$203 \mathrm{H}$. Jing, L. Zhang and H. Wang, in UV-VIS and Photoluminescence Spectroscopy for Nanomaterials Characterization, ed. C. S. S. R. Kumar, Springer Verlag, Berlin, Heidelberg, 1st edn, 2013, Geometrically tunable optical properties of metal nanoparticles, pp. 1-74.

204 M. Dendisová, A. Jeništová, A. Parchaňská-Kokaislová, P. Matějka, V. Prokopec and M. Švecová, Anal. Chim. Acta, 2018, 1031, 1-14.

205 B. Meermann and V. Nischwitz, J. Anal. At. Spectrom., 2018, 33, 1432-1468.

206 A. Brandt, K. Kees and K. Leopold, J. Anal. At. Spectrom., 2020, 35, 2536-2544.

207 H. Lindner, K. H. Loper, D. W. Hahn and K. Niemax, Spectrochim. Acta, Part B, 2011, 66, 179-185.

208 C. Qian, X. Lin, Y. Yang, X. Xiong, H. Wang, E. Li, I. Kaminer, B. Zhang and H. Chen, Phys. Rev. Lett., 2019, 122, 063901.

209 Z. Gong, Y.-L. Pan, G. Videen and C. Wang, J. Quant. Spectrosc. Radiat. Transfer, 2018, 214, 94-119.

210 A. Bogaerts and M. Aghaei, J. Anal. At. Spectrom., 2017, 32, 233-261.

211 M. Aghaei and A. Bogaerts, J. Anal. At. Spectrom., 2016, 31, 631-641.

212 I. Kálomista, A. Kéri and G. Galbács, Talanta, 2017, 172, 147-154.

213 P. M. Carvalho, M. R. Felício, N. C. Santos, S. Gonçalves and M. M. Domingues, Front. Chem., 2018, 6, 237.

214 K. Fukui, Y. Asakuma and K. Maeda, J. Phys.: Conf. Ser., 2010, 215, 012073.

215 A. Oliva, M. Llabrés and J. B. Fariña, Curr. Drug Discovery Technol., 2004, 1, 229-242.

216 R. Xu, Particuology, 2008, 6, 112-115.

217 R. Xu, Particle Characterization: Light Scattering Methods, Springer, Dordrecht, 2000.

218 W. W. Szymanski, A. Nagy and A. Czitrovszky, J. Quant. Spectrosc. Radiat. Transfer, 2009, 110, 918-929.

219 S. K. Brar and M. Verma, Trends Anal. Chem., 2011, 30, 4-17.

220 X. Fan, Z. Shen and B. Luk'yanchuk, Opt. Express, 2010, 18, 24868-24880.

221 D. J. Ross and R. Sigel, Phys. Rev. E: Stat., Nonlinear, Soft Matter Phys., 2012, 85, 056710.

222 H. A. Michelsen, C. Schulz, G. J. Smallwood and S. Will, Prog. Energy Combust. Sci., 2015, 51, 2-48.

223 J. M. Mitrani and M. N. Shneider, Appl. Phys. Lett., 2015, 106, 043102.

224 R. L. VanderWal, G. M. Berger, T. M. Ticich and P. D. Patel, Appl. Opt., 2002, 41, 5678-5690.

225 S. Maffi, F. Cignoli, C. Bellomunno, S. De Iuliis and G. Zizak, Spectrochim. Acta, Part B, 2008, 63, 202-209.

226 T. Lehre, R. Suntz and H. Bockhorn, Proc. Combust. Inst., 2005, 30, 2585-2593.

227 F. Cignoli, C. Bellomunno, S. Maffi and G. Zizak, Appl. Phys. B: Lasers Opt., 2009, 96, 593-599.
228 A. Leipertz and S. Dankers, Part. Part. Syst. Charact., 2003, 20, 81-93.

229 T. A. Sipkens, N. R. Singh, K. J. Daun, N. Bizmark and M. Ioannidis, Appl. Phys. B: Lasers Opt., 2015, 119, 561-575.

230 T. A. Sipkens, R. Mansmann, K. J. Daun, N. Petermann, J. T. Titantah, M. Karttunen, H. Wiggers, T. Dreier and C. Schulz, Appl. Phys. B: Lasers Opt., 2014, 116, 623-636.

231 K. J. Daun, J. T. Titantah and M. Karttunen, Appl. Phys. B: Lasers Opt., 2012, 107, 221-228.

232 R. Sommer and A. Leipertz, Opt. Lett., 2007, 32, 1947-1949.

233 Y. Song, J. Bai, R. Zhang, E. Wu, J. Wang, S. Li, B. Ning, M. Wang, Z. Gao and Y. Peng, Sens. Actuators, B, 2020, 310, 127671.

234 J. P. Schwarz, S. J. Doherty, F. Li, S. T. Ruggiero, C. E. Tanner, A. E. Perring, R. S. Gao and D. W. Fahey, Atmos. Meas. Tech., 2012, 5, 2581-2592.

235 D. F. Andrade, E. R. Pereira-Filho and D. Amarasiriwardena, Appl. Spectrosc. Rev., 2021, 56, 98-114.

236 A. Limbeck, L. Brunnbauer, H. Lohninger, P. Pořízka, P. Modlitbová, J. Kaiser, P. Janovszky, A. Kéri and G. Galbács, Anal. Chim. Acta, 2021, 1147, 72-98.

237 P. Dewalle, J.-B. Sirven, A. Roynette, F. Gensdarmes, L. Golanski and S. Motellier, J. Phys.: Conf. Ser., 2011, 304, 012012.

238 L. J. Radziemski, T. R. Loree, D. A. Cremers and N. M. Hoffman, Anal. Chem., 1983, 55, 1246-1252.

239 P. K. Diwakar, K. H. Loper, A.-M. Matiaske and D. W. Hahn, J. Anal. At. Spectrom., 2012, 27, 1110-1119.

240 D. Diaz, D. W. Hahn and U. Panne, in Laser-Induced Breakdown Spectroscopy, ed J. P. Singh and S. N. Thakur, Elsevier, Amsterdam, 2nd edn, 2020, LIBS for aerosol analysis, pp. 499-535.

241 T. Kuhlen, C. Fricke-Begemann, N. Strauss and R. Noll, Spectrochim. Acta, Part B, 2008, 63, 1171-1176.

242 N. Strauss, C. Fricke-Begemann and R. Noll, J. Anal. At. Spectrom., 2010, 25, 867-874.

243 H. Ji, Y. Ding, L. Zhang, Y. Hu and X. Zhong, Appl. Spectrosc. Rev., 2021, 56, 193-220.

244 Y. Zhang, G. Xiong, S. Li, Z. Dong, S. G. Buckley and S. D. Tse, Combust. Flame, 2013, 160, 725-733.

245 G. Xiong, S. Li, Y. Zhang, S. G. Buckley and S. Tse, J. Anal. At. Spectrom., 2016, 31, 482-491.

246 Y. Zhang, S. Li, Y. Ren, Q. Yao and C. K. Law, Appl. Phys. Lett., 2014, 104, 023115.

247 Y. Zhang, S. Li, Y. Ren, Q. Yao and S. D. Tse, Proc. Combust. Inst., 2015, 35, 3681-3688.

248 Y. Ren, S. Li, Y. Zhang, S. D. Tse and M. B. Long, Phys. Rev. Lett., 2015, 114, 093401.

249 G. Xiong, S. Li and S. D. Tse, Spectrochim. Acta, Part B, 2018, 140, 13-21.

250 S. Y. Chan and N. H. Cheung, Anal. Chem., 2000, 72, 20872092.

251 S. L. Lui and N. H. Cheung, Anal. Chem., 2005, 77, 26172623.

252 X. Wang, F. E. Kruis and P. H. McMurry, Aerosol Sci. Technol., 2007, 39, 611-623. 
253 M. Dienerowitz, M. Mazilu and K. Dholakia, J. Nanophotonics, 2008, 2, 021875.

254 F. J. Fortes, A. Fernández-Bravo and J. J. Laserna, Spectrochim. Acta, Part B, 2014, 100, 78-85.

255 P. Purohit, F. J. Fortes and J. J. Laserna, Spectrochim. Acta, Part B, 2017, 130, 75-81.

256 C. Niu, X. Cheng, T. Zhang, X. Wang, B. He, W. Zhang, Y. Feng, J. Bai and H. Li, Anal. Chem., 2021, 93, 2281-2290.

257 S. T. Järvinen and J. Toivonen, Opt. Express, 2016, 24, 13141323.

258 M. A. Meneses-Nava, I. Rosas-Roman, O. Barbosa-García, M. Rodriguez and J. L. Maldonado, Spectrochim. Acta, Part $B, 2020,168,105855$.

259 P. Modlitbová, P. Pořízka and J. Kaiser, Trends Anal. Chem., 2020, 122, 115729.

260 B. Busser, S. Moncayo, J.-L. Coll, L. Sancey and V. MottoRos, Coord. Chem. Rev., 2018, 358, 70-79.

261 L. Jolivet, M. Leprince, S. Moncayo, L. Sorbier, C.-P. Lienemann and V. Motto-Ros, Spectrochim. Acta, Part $B, 2019,151,41-53$.

262 V. Motto-Ros, L. Sancey, Q. L. Ma, F. Lux, X. S. Bai, X. C. Wang, J. Yu, G. Panczer and O. Tillement, Appl. Phys. Lett., 2012, 101, 223702.

263 V. Motto-Ros, L. Sancey, X. C. Wang, Q. L. Ma, F. Lux, X. S. Bai, G. Panczer, O. Tillement and J. Yu, Spectrochim. Acta, Part B, 2013, 87, 168-174.

264 L. Sancey, V. Motto-Ros, S. Kotb, X. Wang, F. Lux, G. Panczer, J. Yu and O. Tillement, J. Visualized Exp., 2014, 18, 51353.

265 L. Sancey, V. Motto-Ros, B. Busser, S. Kotb, J. M. Benoit, A. Piednoir, F. Lux, O. Tillement, G. Panczer and J. Yu, Sci. Rep., 2014, 4, 6065.

266 L. Sancey, S. Kotb, C. Truillet, F. Appaix, A. Marais and E. Thomas, ACS Nano, 2015, 9, 2477-2488.

267 A. Detappe, S. Kunjachan, L. Sancey, V. Motto-Ros, D. Biancur, P. Drane, R. Guieze, G. M. Makrigiorgos, O. Tillement, R. Langer and R. Berbeco, J. Controlled Release, 2016, 238, 103-113.

268 S. Kunjachan, A. Detappe, R. Kumar, T. I. L. Cameron, D. E. Biancur, V. Motto-Ros, L. Sancey, S. Sridhar, G. M. Makrigiorgos and R. I. Berbeco, Nano Lett., 2015, 15, 7488-7496.

269 Y. Gimenez, B. Busser, F. Trichard, A. Kulesza, J. M. Laurent, V. Zaun, F. Lux, J. M. Benoit, G. Panczer, P. Dugourd, O. Tillement, F. Pelascini, L. Sancey and V. Motto-Ros, Sci. Rep., 2016, 6, 29936.

270 X. Le Guével, M. Henry, V. Motto-Ros, E. Longo, M. I. Montañez, F. Pelascini, O. de La Rochefoucauld, P. Zeitoun, J.-L. Coll, V. Josserand and L. Sancey, Nanoscale, 2018, 10, 18657-18664.

271 L. Krajcarová, K. Novotný, M. Kummerová, J. Dubová, V. Gloser and J. Kaiser, Talanta, 2017, 173, 28-35.

272 P. Modlitbova, K. Novotny, P. Pořizka, J. Klus, H. ZlamalovaGargošova and J. Kaiser, Ecotoxicol. Environ. Saf., 2018, 147, 334-341.
273 P. Modlitbová, P. Pořizka, S. Střítežská, Š. Zezulka, M. Kummerová, K. Novotný and J. Kaiser, Chemosphere, 2020, 251, 126174.

274 P. Modlitbová, A. Hlaváček, T. Švestková, P. Pořízka, L. Šimoníková, K. Novotný and J. Kaiser, Chemosphere, 2019, 225, 723-734.

275 T. Ajtai, Á. Filep, M. Schnaiter, C. Linke, M. Vragel, Z. Bozóki, G. Szabó and T. Leisner, J. Aerosol Sci., 2010, 41, 1020-1029.

276 Z. Bozóki, A. Pogány and G. Szabó, Appl. Spectrosc. Rev., 2011, 46, 1-37.

277 H. Moosmüller, R. K. Chakrabarty and W. P. Arnott, J. Quant. Spectrosc. Radiat. Transfer, 2009, 110, 844-878.

278 J. W. Cremer, P. A. Covert, E. A. Parmentier and R. Signorell, J. Phys. Chem. Lett., 2017, 8, 3398-3403.

279 J. W. Cremer, K. M. Thaler, C. Haisch and R. Signorell, Nat. Commun., 2016, 7, 10941.

280 R. Niessner, Angew. Chem., Int. Ed., 2014, 53, 2-16.

281 M. E. Diveky, S. Roy, J. W. Cremer, G. David and R. Signorell, Phys. Chem. Chem. Phys., 2019, 21, 4721-4731.

282 P. R. Buseck, K. Adachi, A. Gelencsér, E. Tompa and M. Pósfai, Atmos. Chem. Phys. Discuss., 2012, 12, 2482124846.

283 T. Ajtai, Á. Filep, N. Utry, M. Schnaiter, C. Linke, Z. Bozóki, G. Szabó and T. Leisner, J. Aerosol Sci., 2011, 42, 859-866.

284 T. Ajtai, N. Utry, M. Pintér, B. Major, Z. Bozóki and G. Szabó, Atmos. Environ., 2015, 122, 313-320.

285 T. Ajtai, G. Kiss-Albert, N. Utry, Á. Tóth, A. Hoffer, G. Szabó and Z. Bozóki, J. Environ. Sci., 2019, 83, 96-109.

286 F. Grisch and M. Orain, Role of planar laser-induced fluorescence in combustion research, AerospaceLab, 2009, pp. 1-14.

287 Y. Han, Y. Gu, A. C. Zhang and Y.-H. Lo, Lab Chip, 2016, 16, 4639.

288 F. Meng, J. Wang, Q. Ping and Y. Yeo, ACS Nano, 2018, 12, 6458-6468.

289 K. E. Thane, A. M. Davis and A. M. Hoffman, Sci. Rep., 2019, 9, 12295.

290 J. L. Swift and D. T. Cramb, Biophys. J., 2008, 95, 865-876.

291 K. Rashwan, E. Brakke and G. Sereda, Nanotechnol. Rev., 2014, 3, 591-596.

292 H. Kawasaki, Anal. Sci., 2017, 33, 987-988.

293 B. Andreiuk, A. Reisch, M. Lindecker, G. Follain, N. Peyriéras, J. G. Goetz and A. S. Klymchenko, Small, 2017, 13, 1701582.

294 J. Muramoto, T. Inmaru, Y. Nakata, T. Okada and M. Maeda, Appl. Phys. Lett., 2000, 77, 2334.

295 A. Bruno, F. Ossler, C. de Lisio, P. Minutolo, N. Spinelli and A. D'Alessio, Opt. Express, 2008, 16, 5623-5632.

296 C. Weitkamp, Lidar: Range-Resolved Optical Remote Sensing of the Atmosphere, Springer, New York, 2005.

297 S. C. Richardson, M. Mytilinaios, R. Foskinis, C. Kyrou, A. Papayannis, I. Pyrri, E. Giannoutsou and I. D. S. Adamakis, Sci. Total Environ., 2019, 696, 133906.

298 J. A. Huffman, A. E. Perring, N. J. Savage, B. Clot, B. Crouzy, F. Tummon, O. Shoshanim, B. Damit, J. Schneider, V. Sivaprakasam, M. A. Zawadowicz, I. Crawford, 
M. Gallagher, D. Topping, D. C. Doughty, S. C. Hill and Y. Pan, Aerosol Sci. Technol., 2020, 54, 465-495.

299 Z. Rao, D. Hua, T. He, Q. Wang and J. Le, Optik, 2017, 136, 497-502.

300 J. M. Costa-Fernández, M. Menéndez-Miranda, D. BouzasRamos, J. R. Encinar and A. Sanz-Medel, Trends Anal. Chem., 2016, 84, 139-148.

301 A. R. M. Bustos, J. R. Encinar and A. Sanz-Medel, Anal. Bioanal. Chem., 2013, 405, 5637-5643.

302 R. M. Galazzi, K. Chacón-Madrid, D. C. Freitas, L. F. da Costa and M. A. Z. Arruda, Rapid Commun. Mass Spectrom., 2020, 34(S3), e8726.

303 C. Degueldre and P.-Y. Favarger, Colloids Surf., 2003, 217, 137-142.

304 C. Degueldre and P.-Y. Favarger, Talanta, 2004, 62, 10511054.

305 C. Degueldre, P.-Y. Favarger and C. Bitea, Anal. Chim. Acta, 2004, 518, 137-142.

306 C. Degueldre, P.-Y. Favarger and S. Wold, Anal. Chim. Acta, 2006, 555, 263-268.

307 F. Laborda, J. Jiménez-Lamana, E. Bolea and J. R. Castillo, J. Anal. At. Spectrom., 2011, 26, 1362-1371.

308 H. E. Pace, N. J. Rogers, C. Jarolimek, V. A. Coleman, C. P. Higgins and J. F. Ranville, Anal. Chem., 2011, 83, 9361-9369.

309 J. W. Olesik and P. J. Gray, J. Anal. At. Spectrom., 2012, 27, 1143-1155.

310 F. Laborda, E. Bolea and J. Jiménez-Lamana, Anal. Chem., 2014, 86, 2270-2278.

311 K. Flores, R. S. Turley, C. Valdes, Y. Ye, J. Cantu, J. A. Hernandez-Viezcas, J. G. Parsons and J. L. GardeaTorresdey, Appl. Spectrosc. Rev., 2019, 56, 1-26.

312 I. Kálomista, A. Kéri and G. Galbács, J. Anal. At. Spectrom., 2016, 31, 1112-1122.

313 I. Kálomista, A. Kéri, D. Ungor, E. Csapó, I. Dékány, T. Prohaska and G. Galbács, J. Anal. At. Spectrom., 2017, 32, 2455-2462.

314 A. Kéri, I. Kálomista, D. Ungor, Á. Bélteki, E. Csapó, I. Dékány, T. Prohaska and G. Galbács, Talanta, 2018, 179, 193-199.

315 J. Fuchs, M. Aghaei, T. D. Schachel, M. Sperling, A. Bogaerts and U. Karst, Anal. Chem., 2018, 90, 10271-10278.

316 I. Strenge and C. Engelhard, J. Anal. At. Spectrom., 2016, 31, 135-144.

317 A. Hineman and C. Stephan, J. Anal. At. Spectrom., 2014, 29, 1252-1257.

318 M. D. Montano, H. R. Badiei, S. Bazargan and J. F. Ranville, Environ. Sci.: Nano, 2014, 1, 338-346.

319 A. Kaňa, M. Loula, R. Koplík, M. Vosmanská and O. Mestek, Talanta, 2019, 197, 189-198.

320 F. Laborda, A. C. Gimenez-Ingalaturre, E. Bolea and J. R. Castillo, Spectrochim. Acta, Part B, 2019, 159, 105654.

321 S. Lee, X. Bi, R. B. Reed, J. F. Ranville, P. Herckes and P. Westerhoff, Environ. Sci. Technol., 2014, 48, 10291-10300.

322 J. Liu, K. E. Murphy, R. I. MacCuspie and M. R. Winchester, Anal. Chem., 2014, 86, 3405-3414.
323 R. P. Lamsal, G. Jerkiewicz and D. Beauchemin, Anal. Chem., 2016, 88, 10552-10558.

324 R. P. Lamsal, M. S. E. Houache, A. Williams, E. Baranova, G. Jerkiewicz and D. Beauchemin, Anal. Chim. Acta, 2020, 1120, 67-74.

325 S. Gschwind, H. Hagendorfer, D. A. Frick and D. Günther, Anal. Chem., 2013, 85, 5875-5883.

326 S. Gschwind, M. d. L. A. Montes and D. Günther, Anal. Bioanal. Chem., 2015, 407, 4035-4044.

327 B. Ramkorun-Schmidt, S. A. Pergantis, D. EstebanFernández, N. Jakubowski and D. Günther, Anal. Chem., 2015, 87, 8687-8694.

328 L. Hendriks, B. Ramkorun-Schmidt, A. Gundlach-Graham, J. Koch, R. N. Grass, N. Jakubowski and D. Günther, J. Anal. At. Spectrom., 2019, 34, 716-728.

329 D. Rosenkranz, F. L. Kriegel, E. Mavrakis, S. A. Pergantis, P. Reichardt, J. Tentschert, N. Jakubowski, P. Laux, U. Panne and A. Luch, Anal. Chim. Acta, 2020, 1099, 16-25.

330 A. Gundlach-Graham and K. Mehrabi, J. Anal. At. Spectrom., 2020, 35, 1727-1739.

331 R. Peters, Z. Herrera-Rivera, A. Undas, M. van der Lee, H. Marvin, H. Bouwmeester and S. Weigel, J. Anal. At. Spectrom., 2015, 30, 1274-1285.

332 M. Loula, A. Kaňa and O. Mestek, Talanta, 2019, 202, 565571.

333 L. Telgmann, C. D. Metcalfe and H. Hintelmann, J. Anal. At. Spectrom., 2014, 29, 1265-1272.

334 Y. Huang, J. T.-S. Lum and K. S.-Y. Leung, J. Anal. At. Spectrom., 2020, 35, 2148-2155.

335 K. Mehrabi, D. Günther and A. Gundlach-Graham, Environ. Sci.: Nano, 2019, 6, 3349-3358.

336 E. Bolea-Fernandez, D. Leite, A. Rua-Ibarz, L. Balcaen, M. Aramenía, M. Resano and F. Vanhaecke, J. Anal. At. Spectrom., 2017, 32, 2140-2152.

337 M. Tharaud, A. P. Gondikas, M. F. Benedetti, F. von der Kammer, T. Hofmann and G. Cornelis, J. Anal. At. Spectrom., 2017, 32, 1400-1411.

338 S. Candás-Zapico, D. J. Kutscher, M. Montes-Bayón and J. Bettmer, Talanta, 2018, 180, 309-315.

339 A. Rua-Ibarz, E. Bolea-Fernandez, G. Pozo, X. DominguezBenetton, F. Vanhaecke and K. Tirez, J. Anal. At. Spectrom., 2020, 35, 2023-2032.

340 E. Bolea-Fernandez, D. Leite, A. Rua-Ibarz, T. Liu, G. Woods, M. Aramendia, M. Resano and F. Vanhaecke, Anal. Chim. Acta, 2019, 1077, 95-106.

341 A. Praetorius, A. Gundlach-Graham, E. Goldberg, W. Fabienke, J. Navratilova, A. Gondikas, R. Kaegi, D. Günther, T. Hofmann and F. von der Kammer, Environ. Sci.: Nano, 2017, 4, 307-314.

342 S. Naasz, S. Weigel, O. Borovinskaya, A. Serva, C. Cascio, A. K. Undas, F. C. Simeone, H. J. P. Marvin and R. J. B. Peters, J. Anal. At. Spectrom., 2018, 33, 835-845.

343 A. Gondikas, F. von der Kammer, R. Kaegi, O. Borovinskaya, E. Neubauer, J. Navratilova, A. Praetorius, G. Cornelis and T. Hofmann, Environ. Sci.: Nano, 2018, 5, 313-326. 
344 S. Bevers, M. D. Montano, L. Rybicki, T. Hofmann, F. von der Kammer and F. Ranville, Front. Environ. Sci., 2020, 8, 84.

345 S. Tadjiki, M. D. Montano, S. Assemi, A. Barber, J. F. Ranville and R. Beckett, Anal. Chem., 2017, 89, 60566064.

346 N. Joo and H. B. Lim, Bull. Korean Chem. Soc., 2019, 40, 1087-1092.

347 A. Kéri, A. Sápi, D. Ungor, D. Sebők, E. Csapó, Z. Kónya and G. Galbács, J. Anal. At. Spectrom., 2020, 35, 1139-1147.

348 A. Sápi, A. Kéri, I. Kálomista, D. G. Dobó, Á. Szamosvölgyi, K. L. Juhász, Á. Kukovecz, Z. Kónya and G. Galbács, J. Anal. At. Spectrom., 2017, 32, 996-1003.

349 F. Laborda, E. Bolea and J. Jiménez-Lamana, Trends Environ. Anal. Chem., 2016, 9, 15-23.

350 R. J. B. Peters, A. G. Oomen, G. van Bemmel, L. van Vliet, A. K. Undas, S. Munniks, R. L. A. W. Bleys, P. C. Tromp, W. Brand and M. van der Lee, Nanotoxicology, 2020, 14, 420-432.

351 K. Badalova, P. Herbello-Hermelo, P. Bermejo-Barrera and A. Moreda-Piñeiro, J. Trace Elem. Med. Biol., 2019, 54, 55-61.

352 S. Salou, C.-M. Cirtiu, D. Larivière and N. Fleury, Anal. Bioanal. Chem., 2020, 412, 1469-1481.

353 B. Bocca, B. Battistini and F. Petrucci, Talanta, 2020, 220, 121404.

354 E. Leese, J. F. Staff, V. A. Carolan and J. Morton, Ann. Work Exposures Health, 2017, 61, 902-906.

355 X. He, H. Zhang, H. Shi, W. Liu and E. Sahle-Demessie, J. Am. Soc. Mass Spectrom., 2020, 31, 2180-2190.

356 D. M. Peloquin, E. J. Baumann Jr and T. P. Luxton, Chemosphere, 2020, 249, 126173.

357 T. Ishizaka, K. Nagano, I. Tasaki, H. Tao, J.-Q. Gao, K. Harada, K. Hirata, S. Saito, H. Tsujino, K. Higashisaka and Y. Tsutsumi, Nanoscale Res. Lett., 2019, 14, 180.

358 F. Aureli, M. Ciprotti, M. D'Amato, E. do N. da Silva, S. Nisi, D. Passeri, A. Sorbo, A. Raggi, M. Rossi and F. Cubadda, Nanomaterials, 2020, 10, 888.

359 S. Kuehr, R. Kaegi, D. Maletzki and C. Schlechtriem, Cemosphere, 2021, 263, 127961.

360 F. Dang, Q. Wang, W. Cai, D. Zhou and B. Xing, Nanotoxicology, 2020, 14, 654-666.

361 M. Hayder, J. Wojcieszek, M. Asztemborska, Y. Zhou and L. Ruzik, J. Sci. Food Agric., 2020, 100, 4950-4958.

362 Y. Wu, L. Yang, H. Gong, F. Dang and D.-M. Zhou, Environ. Pollut., 2020, 260, 113969.

363 Q. Zhou, L. Liu, N. Liu, B. He, L. Hu and L. Wang, Ecotoxicol. Environ. Saf., 2020, 198, 110670.

364 L. Xu, Z. Wang, J. Zhao, M. Lin and B. Xing, Environ. Pollut., 2020, 260, 114043.

365 D. Ojeda, M. V. Taboada-López, E. Bolea, J. Pérez-Arantegui, P. Bermejo-Barrera, A. Moreda-Piñeiro and F. Laborda, Anal. Chim. Acta, 2020, 1122, 20-30.

366 E. Verleysen, N. Waegeneers, F. Brassinne, S. De Vos, I. O. Jimenez, S. Mathioudaki and J. Mast, Nanomaterials, 2020, 10, 592.

367 O. Geiss, I. Bianchi, C. Senaldi, G. Bucher, E. Verleysen, N. Waegeneers, F. Brassinne, J. Mast, K. Loeschner,
J. Vidmar, F. Aureli, F. Cubadda, A. Raggi, F. Iacoponi, R. Peters, A. Undas, A. Müller, A.-K. Meinhardt, E. Walz, V. Gräf and J. Borrero-Moreno, Food Control, 2021, 120, 107550.

368 K. Loeschner, M. Correia, C. L. Chaves, I. Rokkjær and J. J. Sloth, Food Addit. Contam., Part A, 2017, 35, 86-93.

369 Y. Dan, H. Shi, C. Stephan and X. Liang, Microchem. J., 2015, 122, 119-126.

370 M. Correia, T. Uusimäki, A. Philippe and K. Loeschner, Separations, 2018, 5, 56.

371 A. Laycock, M. D. Wright, I. Römer, A. Buckley and R. Smith, Atmos. Environ.: X, 2020, 8, 100079.

372 A. Mackevica, M. E. Olsson and S. F. Hansen, J. Hazard. Mater., 2017, 322, 270-275.

373 A. Mackevica, M. E. Olsson and S. F. Hansen, J. Nanopart. Res., 2018, 20, 6.

374 S. A. Ntim, S. Norris, K. Scott, T. A. Thomas and G. O. Noonan, Food Control, 2018, 87, 31-39.

375 A. S. Adeleye, E. A. Oranu, M. Tao and A. A. Keller, Water Res., 2016, 102, 374-382.

376 A. Azimzada, J. M. Farner, M. Hadioui, C. Liu-Kang, I. Jreije, N. Tufenkji and K. J. Wilkinson, Environ. Sci.: Nano, 2020, 7, 139-148.

377 D. P. Martin, N. L. Melby, S. M. Jordan, A. J. Bednar, A. J. Kennedy, M. E. Negrete, M. A. Chappell and A. R. Poda, Chemosphere, 2016, 162, 222-227.

378 R. S. Lankone, K. Challis, L. Pourzahedi, D. P. Durkin, Y. Bi, Y. Wang, M. A. Garland, F. Brown, K. Hristovski, R. L. Tanguay, P. Westerhoff, G. Lowry, L. M. Gilbertson, J. Ranville and D. H. Fairbrother, Sci. Total Environ., 2019, 668, 234-244.

379 B. Bocca, E. Sabbioni, I. Mičetić, A. Alimonti and F. Petrucci, J. Anal. At. Spectrom., 2017, 32, 616-628.

380 B. Battistini, F. Petrucci, I. De Angelis, C. M. Failla and B. Bocca, Chemosphere, 2020, 245, 125667.

381 L. Mueller, H. Traub, N. Jakubowski, D. Drescher, V. I. Baranov and J. Kneipp, Anal. Bioanal. Chem., 2014, 406, 6963-6977.

382 S. Theiner, K. Loehr, G. Koellensperger, L. Mueller and N. Jakubowski, J. Anal. At. Spectrom., 2020, 35, 1784-1813.

383 M. Corte-Rodríguez, R. Álvarez-Fernández, P. GarcíaCancela, M. Montes-Bayón and J. Bettmer, Trends Anal. Chem., 2020, 132, 116042.

384 S. Miyashita, A. S. Groombridge, S. Fujii, A. Minoda, A. Takatsu, A. Hioki, K. Chiba and K. Inagaki, J. Anal. At. Spectrom., 2014, 29, 1598-1606.

385 J. T.-S. Lum and K. S.-Y. Leung, Anal. Chim. Acta, 2019, 1061, 50-59.

386 Y. Cao, J. Feng, L. Tang, C. Yu, G. Mo and B. Deng, Talanta, 2020, 206, 120174.

387 L. Á.-F. García, M. Corte-Rodríguez, M. Macke, K. L. LeBlanc, Z. Mester, M. Montes-Bayón and J. Bettmer, Analyst, 2020, 145, 1457-1465.

388 S. Meyer, A. Lopez-Serrano, H. Mitze, N. Jakubowski and T. Schwerdtle, Metallomics, 2018, 10, 73-76. 
389 B. Gomez-Gomez, M. Corte-Rodríguez, M. T. Perez-Corona, J. Bettmer, M. Montes-Bayón and Y. Madrid, Anal. Chim. Acta, 2020, 1128, 116-128.

390 Y. Cao, J. Feng, L. Tang, G. Mo, W. Mo and B. Deng, Spectrochim. Acta, Part B, 2020, 166, 105797.

391 Z. Huang, C. Wang, R. Liu, Y. Su and Y. Lv, Anal. Chem., 2020, 92, 2876-2881.

392 Z. Huang, Z. Li, M. Jiang, R. Liu and Y. Lv, Anal. Chem., 2020, 92, 16105-16112.

393 J. Nelson, M. Yamanaka, F. A. Lopez-Linares, L. Poirier and E. Rogel, Energy Fuels, 2017, 31, 11971-11976.

394 D. Ruhland, K. Nwoko, M. Perez, J. Feldmann and E. M. Krupp, Anal. Chem., 2019, 91, 1164-1170.

395 R. d. Heringer and J. F. Ranville, Forensic Sci. Int., 2018, 288, e20-e25.

396 R. S. Pappas, N. Gray, M. Halstead, L. Valentin-Blasini and C. Watson, J. Anal. Toxicol., 2020, bkaa088.

397 N. Shin, K. Velmurugan, C. Su, A. K. Bauer and C. S. J. Tsai, Environ. Sci.: Processes Impacts, 2019, 21, 1342-1352.

398 Q. Chan, M. Entezarian, J. Zhou, R. Osterloh, Q. Huang, M. Ellefson, B. Mader, Y. Liu and M. Swierczek, J. Membr. Sci., 2020, 599, 117822.

399 D. Pozebon, G. L. Scheffler and V. L. Dressler, J. Anal. At. Spectrom., 2017, 32, 890-919.

400 M. Martinez and M. Baudelet, Anal. Bioanal. Chem., 2020, 412, 27-36.

401 A. Sajnóg, A. Hanć and D. Barałkiewicz, Talanta, 2018, 182, 92-110.

402 D. Drescher, C. Giesen, H. Traub, U. Panne, J. Kneipp and N. Jakubowski, Anal. Chem., 2012, 84, 9684-9688.

403 N. N. Mahmoud, A. M. Alkilany, D. Dietrich, U. Karst, A. G. Al-Bakri and E. A. Khalil, J. Colloid Interface Sci., 2017, 503, 95-102.

404 I.-L. Hsiao, F. S. Bierkandt, P. Reichardt, A. Luch, Y.-J. Huang, N. Jakubowski, J. Tentschert and A. Haase, J. Nanobiotechnol., 2016, 14, 50.

405 S. G. Elci, Y. Jiang, B. Yan, S. T. Kim, K. Saha, D. F. Moyano, G. Y. Tonga, L. C. Jackson, V. M. Rotello and R. W. Vachet, ACS Nano, 2016, 10, 5536-5542.

406 S. G. Elci, G. Y. Tonga, B. Yan, S. T. Kim, C. S. Kim, Y. Jiang, K. Saha, D. F. Moyano, A. L. M. Marsico, V. M. Rotello and R. W. Vachet, ACS Nano, 2017, 11, 7424-7430.

407 T. Büchner, D. Drescher, H. Traub, P. Schrade, S. Bachmann, N. Jakubowski and J. Kneipp, Anal. Bioanal. Chem., 2014, 406, 7003-7014.

408 S. Yamashita, Y. Yoshikuni, H. Obayashi, T. Suzuki, D. Green and T. Hirata, Anal. Chem., 2019, 91, 4544-4551.

409 J. Wojcieszek, J. Jiménez-Lamana, K. Bierła, L. Ruzik, M. Asztemborska, M. Jarosz and J. Szpunar, Sci. Total Environ., 2019, 683, 284-292.

410 O. Reifschneider, A. Vennemann, G. Buzanich, M. Radtke, U. Reinholz, H. Riesemeier, J. Hogeback, C. Köppen, M. Großgarten, M. Sperling, M. Wiemann and U. Karst, Chem. Res. Toxicol., 2020, 33, 1250-1255.

411 V. M. Neves, G. M. Heidrich, E. S. Rodrigues, M. S. P. Enders, E. I. Muller, F. T. Nicoloso, H. W. P. de
Carvalho and V. L. Dressler, Environ. Sci. Technol., 2019, 53, 10827-10834.

412 D. Metarapi, M. Šala, K. Vogel-Mikuš, V. S. Šelih and J. T. van Elteren, Anal. Chem., 2019, 91, 6200-6205.

413 J. A. Ko, N. Furuta and H. B. Lim, Anal. Chim. Acta, 2019, 1069, 28-35.

414 L. Zheng, L. Feng, J.-W. Shi, H. Chen, B. Wang, M. Wang, H. Wang and W. Feng, Anal. Chem., 2020, 92, 14339-14345.

415 S. Böhme, H. J. Stärk, D. Kühnel and T. Reemtsma, Anal. Bioanal. Chem., 2015, 407, 5477-5485.

416 J. Touriniemi, T. R. Holbrook, G. Cornelis, M. Schmitt, H.-J. Starck and S. Wagner, J. Anal. At. Spectrom., 2020, 35, 1678-1686.

417 D. Metarapi and J. T. van Elteren, J. Anal. At. Spectrom., 2020, 35, 784-793.

418 J.-F. Li, C.-Y. Li and R. F. Aroca, Chem. Soc. Rev., 2017, 46, 3962-3979.

419 F. Horak, A. Nagl, K. Föttinger and A. Limbeck, Microchim. Acta, 2020, 187, 641.

420 L. Zheng, Y. Sang, R. Luo, B. Wang, F. Yi, M. Wang and W. Feng, J. Anal. At. Spectrom., 2019, 34, 915-921.

421 T. Van Acker, E. Bolea-Fernandez, E. De Vlieghere, J. Gao, O. De Weverbd and F. Vanhaecke, J. Anal. At. Spectrom., 2019, 34, 1846-1855.

422 L. Trapiella-Alfonso, A. R. Montoro, J. R. Encinar, J. M. Costa-Fernandez, R. Pereiro and A. Sanz-Medel, Nanoscale, 2011, 3, 954-957.

423 P. Paydary and P. Larese-Casanova, Int. J. Environ. Anal. Chem., 2015, 95, 1450-1470.

424 L. Pitkänen and A. M. Striegel, Trends Anal. Chem., 2016, 80, 311-320.

425 K. Tiede, A. B. A. Boxall, X. Wang, D. Gore, D. Tiede, M. Baxter, H. David, S. P. Teare and J. Lewis, J. Anal. At. Spectrom., 2010, 25, 1149-1154.

426 S. A. Pergantis, T. L. Jones-Lepp and E. M. Heithmar, Anal. Chem., 2012, 84, 6454-6462.

427 M. Roman, C. Rigo, H. Castillo-Michel, I. Munivrana, V. Vindigni, I. Mičetić, F. Benetti, L. Manodori and W. R. L. Cairns, Anal. Bioanal. Chem., 2016, 408, 5109-5124.

428 L. Pitkänen, A. R. M. Bustos, K. E. Murphy, M. R. Winchester and A. M. Striegel, J. Chromatogr. A, 2017, 1511, 59-67.

429 B. Franze and C. Engelhard, Anal. Chem., 2014, 86, 57135720.

430 J. Kruszewska, D. Kulpińska, I. Grabowska-Jadach and M. Matczuk, Metallomics, 2020, 12, 408-415.

431 J. Kruszewska, J. Sikorski, J. Samsonowicz-Górski and M. Matczuk, Anal. Bioanal. Chem., 2020, 412, 8145-8153.

432 J. Legat, M. Matczuk, A. Timerbaev and M. Jarosz, Chromatographia, 2017, 80, 1695-1700.

433 F. Grønbæk-Thorsen, R. H. Hansen, J. Østergaard, B. Gammelgaard and L. H. Møller, Anal. Bioanal. Chem., 2021, 413, 2247-2255.

434 L. Liu, B. He, Q. Liu, Z. Yun, X. Yan, Y. Long and G. Jiang, Angew. Chem., Int. Ed., 2014, 53, 14476-14479.

435 J. Gigault and V. A. Hackley, Anal. Chim. Acta, 2013, 763, 5766. 
436 J. Gigault, J. M. Pettibone, C. Schmitt and V. A. Hackley, Anal. Chim. Acta, 2014, 809, 9-24.

437 M. Menendez-Miranda, M. T. Fernandez-Arguelles, J. M. Costa-Fernandez, J. Ruiz and A. Sanz-Medel, Anal. Chim. Acta, 2014, 839, 8-13.

438 M. Menendez-Miranda, J. Ruiz, J. M. Costa-Fernández and A. Sanz-Medel, J. Chromatogr. A, 2015, 1422, 247-252.

439 S. Dubascoux, I. Le Hecho, M. Hassellöv, F. Von Der Kammer, M. P. Gautier and G. Lespes, J. Anal. At. Spectrom., 2010, 25, 613-623.

440 A. J. Bednar, A. R. Poda, D. M. Mitrano, A. J. Kennedy, E. P. Gray, J. F. Ranville, C. A. Hayes, F. H. Crocker and J. A. Steevens, Talanta, 2013, 104, 140-148.

441 B. Bocca, S. Caimi, O. Senofonte, A. Alimonti and F. Petrucci, Sci. Total Environ., 2018, 630, 922-930.

442 J. Yang, P. Tan, T. Huang and V. Nischwitz, Anal. Chim. Acta, 2020, 1093, 16-27.

443 S. Elzey, T. De-Ha, L. L. Yu, M. R. Winchester, M. E. Kelley and V. A. Hackley, Anal. Bioanal. Chem., 2013, 405, 22792288.

444 J. Tan, Y. Yang, H. E. Hadri, M. Li, V. A. Hackley and M. R. Zachariah, Analyst, 2019, 144, 2275-2283.

445 D.-H. Tsai, T. J. Cho, S. R. Elzey, J. C. Gigault and V. A. Hackley, Nanoscale, 2013, 5, 5390-5395.

446 F. Hillenkamp and J. Peter-Katalinic, MALDI MS: A Practical Guide to Instrumentation, Methods, and Applications, Wiley-VCH Verlag GmbH \& Co. KGaA, Weinheim, 2nd edn, 2014.

447 Z. Guo, A. A. A. Ganawi, Q. Liu and L. He, Anal. Bioanal. Chem., 2006, 384, 584-592.

448 H. N. Abdelhamid, Handbook of Smart Materials in Analytical Chemistry, ed. M. de la Guardia and F. A. Esteve-Turrillas, John Wiley \& Sons, Inc., Hoboken, New Jersey, 1st edn, 2019, Nanoparticles Assisted Laser Desorption/Ionization Mass Spectrometry.

449 R. A. Picca, C. D. Calvano, N. Cioffi and F. Palmisano, Nanomaterials, 2017, 7, 75.

450 P. T. Steele, H. J. Tobias, D. P. Fergenson, M. E. Pitesky, J. M. Horn, G. A. Czerwieniec, S. C. Russell, C. B. Lebrilla, E. E. Gard and M. Frank, Anal. Chem., 2003, 75, 5480-5487.

451 M. A. Stowers, A. L. van Wuijckhuijse, J. C. M. Marijnissen, C. E. Kientz and T. Ciach, Appl. Opt., 2006, 45, 8531-8536.

452 W. A. Kleefsman, M. A. Stowers, P. J. T. Verheijen and J. C. M. Marijnissen, KONA Powder Part. J., 2008, 26, 205214.

453 K.-P. Hinz and B. Spengler, J. Mass Spectrom., 2007, 42, 843860.

454 R. Knoblauch and C. D. Geddes, in Reviews in Plasmonics 2017, ed. C. D. Geddes, Springer Nature Switzerland, Cham, 1st edn, 2017, Review of Advances in MetalEnhanced Fluorescence, pp. 253-283.

455 K. M. Harkness, B. C. Hixson, L. S. Fenn, B. N. Turner, A. C. Rape, C. A. Simpson, B. J. Huffman, T. C. Okoli, J. A. McLean and D. E. Cliffel, Anal. Chem., 2010, 82, 9268-9274.
456 S. N. Merz, Z. J. Farrell, J. Pearring, E. Hoover, M. Kester, S. A. Egorov, D. L. Green and K. H. DuBay, ACS Nano, 2018, 12, 11031-11040.

457 Z. Luo, J. Hou, L. Menin, Q. K. Ong and F. Stellacci, Angew. Chem., 2017, 129, 13706-13710.

458 Z. Luo, Y. Zhao, T. Darwish, Y. Wang, J. Hou and F. Stellacci, Nat. Commun., 2018, 9, 4478.

459 S. Liao, Z. Luo, J. B. Metternich, R. Zenobi and F. Stellacci, Nanoscale, 2020, 12, 22639-22644.

460 S. A. Maier, Plasmonics: Fundamentals and Applications, Springer, New York, 1st edn, 2007.

461 R. W. Wood, Philos. Mag., 1902, 4, 396-402.

462 G. Mie, Ann. Phys., 1908, 330, 377-445.

463 E. Kretschmann and H. Raether, Z. Naturforsch., A: Phys., Phys. Chem., Kosmophys., 1968, 23, 2135-2136.

464 M. Fleischmann, J. P. Hendra and A. J. McQuillan, Chem. Phys. Lett., 1974, 26, 163-166.

465 D. A. Weitz, S. Garoff, J. I. Gersten and A. Nitzan, J. Chem. Phys., 1983, 78, 5324-5338.

466 U. Dürig, D. W. Pohl and F. Rohner, J. Appl. Phys., 1986, 59, 3318-3327.

467 M. D. Sonntag, E. A. Pozzi, N. Jiang, M. C. Hersam and R. P. Van Duyne, J. Phys. Chem. Lett., 2014, 5, 3125-3130.

468 K. A. Willets and R. P. Van Duyne, Annu. Rev. Phys. Chem., 2007, 58, 267-297.

469 T. Ohta, M. Ito, T. Kotani and T. Hattori, Appl. Spectrosc., 2009, 63, 555-558.

470 J. Liu, H. He, D. Xiao, S. Yin, W. Ji, S. Jiang, D. Luo, B. Wang and Y. Liu, Materials, 2018, 11, 1833.

471 J. Krajczewski, K. Kołąaja and A. Kudelski, RSC Adv., 2017, 7, 17559-17576.

472 N. G. Khlebtsov and L. A. Dykman, J. Quant. Spectrosc. Radiat. Transfer, 2010, 111, 1-35.

473 A. L. Gonzalez, C. Noguez and A. S. Barnard, J. Mater. Chem. C, 2013, 1, 3150-3157.

474 M. Li, S. K. Cushing and N. Wu, Analyst, 2015, 140, 386-406.

475 A. W. Powell, M. B. Wincott, A. A. R. Watt, H. E. Assender and J. M. Smith, J. Appl. Phys., 2013, 113, 184311.

476 A. R. Tao, S. Habas and P. Yang, Small, 2008, 4, 310-325.

477 N. W. Ashcroft and D. N. Mermin, Solid State Physics, Cengage Learning, Boston, Massachusetts, 1st edn, 1976.

478 J. R. Lackowicz, Plasmonics, 2006, 1, 5-33.

479 T. Sarkar, M. N. Abdallah, M. Salazar and W. Dyab, IEEE Trans. Antennas Propag., 2017, 59, 77-93.

480 E. Le Ru and P. Etchegoin, Principles of Surface-Enhanced Raman Spectroscopy, Elsevier, Amsterdam, 1st edn, 2008.

481 K. M. Mayer and J. H. Hafner, Chem. Rev., 2011, 111, 38283857.

482 P. D. Nallathamby, T. Huang and X. H. N. Xu, Nanoscale, 2010, 2, 1715-1722.

483 S. Wang, W. Xi, F. Cai, X. Zhao, Z. Xu, J. Qian and S. He, Theranostics, 2015, 5, 251-266.

484 E. Petryayeva and U. J. Krull, Anal. Chim. Acta, 2011, 706, 824.

485 M. Wang, M. Ye, J. Iocozzia, C. Lin and Z. Lin, Adv. Sci., 2016, 3, 1600024.

486 A. Bonyár, ACS Appl. Nano Mater., 2020, 3, 8506-8521. 
487 S. Kasani, K. Curtin and N. Wu, Nanophotonics, 2019, 8, 2065-2089.

488 J. N. Anker, W. P. Hall, O. Lyandres, N. C. Shah, J. Zhao and V. Duyne, Nat. Mater., 2008, 7, 442-453.

489 J. Cao, T. Sun and K. T. V. Grattan, Sens. Actuators, B, 2014, 195, 332-351.

490 B. Sepúlveda, P. C. Angelomé, L. M. Lechuga and L. M. LizMarzán, Nano Today, 2009, 4, 244-251.

491 S. Unser, I. Bruzas, J. He and L. Sagle, Sensors, 2015, 15, 15684-15716.

492 A. Yanase and H. Komiyama, Surf. Sci., 1992, 264, 147-156. 493 A. Farnood, M. Ranjbar and H. Salamati, Int. J. Hydrogen Energy, 2020, 1, 1158-1169.

494 J. M. Bingham, J. N. Anker, L. E. Kreno and R. P. Van Duyne, J. Am. Chem. Soc., 2010, 132, 17358-17359.

495 T. Karakouz, A. Vaskevich and I. Rubinstein, J. Phys. Chem. B, 2008, 112, 14530-14538.

496 C. S. Cheng, Y. Q. Chen and C. Lu, Talanta, 2007, 73, 358365.

497 M. Potara, A. M. Gabudean and S. Astilean, J. Mater. Chem., 2011, 21, 3625-3633.

498 W. Zheng, H. Li, W. Chen, J. Ji and X. Jiang, Anal. Chem., 2016, 88, 4140-4146.

499 H. Niu, S. Wang, Z. Zhou, Y. Ma, X. Ma X and Y. Cai, Anal. Chem., 2014, 86, 4170-4177.

500 A. Laromaine, L. Koh, M. Murugesan, R. V. Ulijn and M. M. Stevens, J. Am. Chem. Soc., 2007, 129, 4156-4157.

501 T. Lin, W. Chen, Y. Shiang, C. Huang and H. Chang, Biosens. Bioelectron., 2011, 29, 204-209.

502 L. Guo, Y. Xu, A. R. Ferhan, G. Chen and D. Kim, J. Am. Chem. Soc., 2013, 135, 12338-12345.

503 C. M. Niemeyer, Angew. Chem., Int. Ed., 2001, 40, 41284158.

504 M. López-García, J. F. Galisteo-López, A. Blanco, J. SánchezMarcos, C. López and A. García-Martín, Small, 2010, 6, 1757-1761.

505 D. Rout and R. Vijaya, J. Appl. Phys., 2016, 119, 023108.

506 Y. Vasquez, M. Kolle, L. Mishchenko, B. D. Hatton and J. Aizenberg, ACS Photonics, 2014, 1, 53-60.

507 M. Proença, M. S. Rodrigues, J. Borges and F. Vaz, Coatings, 2019, 9, 337.

508 M. Proença, M. S. Rodrigues, J. Borges and F. Vaz, Anal. Chem., 2020, 92, 4349-4356.

509 X. Li, L. Peng, J. Cui, W. Li, C. Lin, D. Xu, T. Tian, G. Zhang and D. Zhang, Small, 2012, 8, 612-618.

510 Z. Xie, K. Cao, Y. Zhao, L. Bai, H. Gu, H. Xu and Z. Z. Gu, Adv. Mater., 2014, 26, 2413-2418.

511 D. P. Puzzo, A. C. Arsenault, I. Manners and G. A. Ozin, Angew. Chem., 2009, 48, 943-947.

512 L. He, M. Wang, J. Ge and Y. Yin, Acc. Chem. Res., 2012, 45, 1431-1440.

513 M. I. S. M. H. Tan, A. F. Omar, M. Rashid and U. Hashim, Results Phys., 2019, 14, 102497.

514 R. Buonsanti, A. Llordes, S. Aloni, B. A. Helms and D. J. Milliron, Nano Lett., 2011, 11, 4706-4710.

515 P. S. S. dos Santos, J. M. M. M. de Almeida, I. PastorizaSantos and L. C. C. Coelho, Sensors, 2021, 21, 2111.
516 T. R. Gordon, T. Paik, D. R. Klein, G. V. Naik, H. Caglayan, A. Boltasseva and C. B. Murray, Nano Lett., 2013, 13, 28572863.

517 M. A. Hosseini, M. Ranjbar and M. Sajadi, Phys. Lett. A, 2020, 384, 126079.

518 K. Manthiram and A. P. Alivisatos, J. Am. Chem. Soc., 2012, 134, 3995-3998.

519 L. A. Sweatlock and K. Diest, Opt. Express, 2012, 20, 87008709.

520 M. Braik, I. Sow, J. Nelayah, A. Belkhir, M. Faustini, S. Mercone, S. Nowak, P. Decorse, J.-Y. Piquemal and N. Felidj, Nanoscale, 2021, 13, 2639-2647.

521 D. Chen, J. Li, C. Shi, X. Du, N. Zhao, J. Sheng and S. Liu, Chem. Mater., 2007, 19, 3399-3405.

522 Z. Lu, M. D. Prouty, Z. Guo, V. O. Golub, C. S. Kumar and Y. M. Lvov, Langmuir, 2005, 21, 2042-2050.

523 J. Popp and T. Mayerhöfer, Micro-Raman Spectroscopy: Theory and Application, De Gruyter, Berlin, 1st edn, 2020.

524 E. Smith and G. Dent, Modern Raman Spectroscopy: A Practical Approach, John Wiley \& Sons, Hoboken, New Jersey, 1st edn, 2013.

525 M. G. Albrecht and J. A. Creighton, J. Am. Chem. Soc., 1977, 99, 5215-5217.

526 M. Moskovits, J. Chem. Phys., 1978, 69, 4159-4161.

527 M. Moskovits, Rev. Mod. Phys., 1985, 57, 783-826.

528 Y. Cheong, Y. J. Kim, H. Kang, S. Choi and H. J. Lee, Spectrochim. Acta, Part A, 2017, 183, 53-59.

529 P. Mao, C. Liu, G. Favraud, Q. Chen, M. Han, A. Fratalocchi and S. Zhang, Nat. Commun., 2018, 9, 5428.

530 J.-F. Li, Y.-F. Huang, S. Duan, R. Pang, D.-Y. Wu, B. Ren, X. Xu and Z.-Q. Tian, Phys. Chem. Chem. Phys., 2010, 12, 2493-2502.

531 S. Nie and S. R. Emory, Science, 1997, 275, 1102-1106.

532 A. M. Polubotko and V. P. Chelibanov, Opt. Spectrosc., 2017, 122, 937-943.

533 S.-Y. Ding, E.-M. You, Z.-Q. Tian and M. Moskovits, Chem. Soc. Rev., 2017, 46, 4042-4076.

534 S. M. Morton and L. Jensen, J. Am. Chem. Soc., 2009, 131, 4090-4098.

535 A. Otto, J. Raman Spectrosc., 2005, 36, 497-509.

536 R. Pilot, R. Signorini, C. Durante, L. Orian, M. Bhamidipati and L. Fabris, Biosensors, 2019, 9, 57.

537 M. Fan, G. F. S. Andrade and A. G. Brolo, Anal. Chim. Acta, 2020, 1097, 1-29.

538 M. Li, S. K. Cushing, J. Zhang, J. Lankford, Z. P. Aguilar, D. Ma and N. Wu, Nanotechnology, 2012, 23, 115501.

539 C. David, N. Guillot, H. Shen, T. Toury and M. L. de la Chapelle, Nanotechnology, 2010, 21, 475501.

540 I. Bruzas, W. Lum, Z. Gorunmez and L. Sagle, Analyst, 2018, 143, 3990-4008.

541 K. Kim, J.-Y. Choi, H. B. Lee and K. S. Shin, J. Chem. Phys., 2011, 135, 124705.

542 Q. Zhang, Y. H. Lee, I. Y. Phang, C. K. Lee and X. Y. Ling, Small, 2014, 10, 2703-2711.

543 I. Rigó, M. Veres, Zs. Pápa, L. Himics, R. Öcsi, O. Hakkel and P. Fürjes, J. Quant. Spectrosc. Radiat. Transfer, 2020, 253, 107128. 
544 Z. Ye, C. Li, Q. Chen, Y. Xu and S. E. J. Bell, Nanoscale, 2021, 13, 5937-5953.

545 K. Kim, H. Han, I. Choi, C. Lee, S. G. Hong, S.-H. Suh, L. P. Lee and T. Kang, Nat. Commun., 2013, 4, 2182.

546 W. Luo, C. Wu, S. Huang, X. Luo, R. Yuan and X. Yang, Anal. Chem., 2020, 92, 15573-15578.

547 L. Zhang, F. Liu, Y. Zou, X. Hu, S. Huang, Y. Xu, L. Zhang, Q. Dong, Z. Liu, L. Chen, Z. Chen and W. Tan, Anal. Chem., 2018, 90, 11183-11187.

548 Y. Li, D. Tian, Y. Ding, G. Yang, K. Liu, C. Wang and X. Han, Appl. Spectrosc. Rev., 2018, 53, 1-35.

549 A. De Giacomo, M. Dell'Aglio, R. Gaudiuso, C. Koral and G. Valenza, J. Anal. At. Spectrom., 2016, 31, 1566-1573.

550 A. De Giacomo, C. Koral, G. Valenza, R. Gaudiuso and M. Dell'Aglio, Anal. Chem., 2016, 88, 5251-5257.

551 A. De Giacomo, R. Gaudiuso, C. Koral, M. Dell'Aglio and O. De Pascale, Spectrochim. Acta, Part B, 2014, 98, 19-27.

552 A. De Giacomo, R. Gaudiuso, C. Koral, M. Dell'Aglio and O. De Pascale, Anal. Chem., 2013, 85, 10180-10187.

553 C. Koral, M. Dell'Aglio, R. Gaudiuso, R. Alrifai, M. Torelli and A. De Giacomo, Talanta, 2018, 182, 253-258.

554 D. A. Rusak, T. P. Anthony and Z. T. Bell, Rev. Sci. Instrum., 2015, 86, 116106.

555 X. Wen, Q. Lin, G. Niu, Q. Shi and Y. Duan, Appl. Opt., 2016, 55, 6706-6712.

556 X. Zhao, C. Zhao, X. Du and D. Dong, Sci. Rep., 2019, 9, 906.

557 Z. Abdel-Salam, S. M. I. Alexeree and M. A. Harith, Spectrochim. Acta, Part B, 2018, 149, 112-117.

558 Z. A. Abdel-Salam, V. Palleschi and M. A. Harith, J. Adv. Res., 2019, 17, 65-72.

559 M. Dell'Aglio, R. Alrifai and A. De Giacomo, Spectrochim. Acta, Part B, 2018, 148, 105-112.

560 J. Liu, Z. Hou, T. Li, Y. Fu and Z. Wang, J. Anal. At. Spectrom., 2020, 35, 2274-2281.

561 R. Gaudiuso, C. Koral, M. Dell'Aglio, O. De Pascale and A. de Giacomo in Nano-Optics: Principles Enabling Basic Research and Applications. NATO Science for Peace and Security Series B: Physics and Biophysics, ed. B. Di Bartolo, J. Collins and L. Silvestri, Springer, Dordrecht, 2017, Fundamental Study and Analytical Applications of Nanoparticle-Enhanced Laser-Induced Breakdown Spectroscopy (NELIBS) of Metals, Semiconductors and Insulators, pp. 505-506.

562 A. Mangone, F. Mastrorocco, L. Giannossa, R. Comparelli, M. Dell'Aglio and A. De Giacomo, Spectrochim. Acta, Part $B, 2020,163,105731$.

563 M. Holá, Z. Salajková, A. Hrdlička, J. Ondráček, K. Novotný, D. Pavliňák, M. Vojtíšek-Lom, L. Čelko, P. Pořízka, V. Kanický, D. Prochazka, J. Novotný and J. Kaiser, J. Anal. At. Spectrom., 2020, 35, 2893-2900.

564 J. W. Liaw, H. Y. Tsai and C. H. Huang, Plasmonics, 2012, 7, 543-553.

565 C. D. Geddes, Phys. Chem. Chem. Phys., 2013, 15, 19537.

566 K. Aslan, Z. Leonenko, J. R. Lakowicz and C. D. Geddes, J. Fluoresc., 2005, 15, 643-654.

567 M. A. Badshah, N. Y. Koh, A. W. Zia, N. Abbas, Z. Zahra and M. W. Saleem, Nanomaterials, 2020, 10, 1749.
568 M. Hlaing, B. Gebear-Eigzabher, A. Roa, A. Marcano, D. Radu and C. Y. Lai, Opt. Mater., 2016, 58, 439-444.

569 M. A. Badshah, J. Ju, X. Lu, N. Abbas and S. Kim, Sens. Actuators, B, 2018, 274, 451-457.

570 A. Puchkova, C. Vietz, E. Pibiri, B. Wünsch, M. S. Paz, G. P. Acuna and P. Tinnefeld, Nano Lett., 2015, 15, 83548359.

571 N. S. Abadeer, M. R. Brennan, W. L. Wilson and C. J. Murphy, ACS Nano, 2014, 8, 8392-8406.

572 G. A. Jones and D. S. Bradshaw, Front. Phys., 2019, 7, 100.

573 J. B. Khurgin and G. Sun, J. Opt. Soc. Am. B, 2009, 26, B83B95.

574 P. K. Jain, K. S. Lee, I. H. El-Sayed and M. A. El-Sayed, J. Phys. Chem. B, 2006, 110, 7238-7248.

575 M. Shabaninezhad, J. Chem. Phys., 2019, 150, 144116.

576 J. R. Lakowicz, K. Ray, M. Chowdhury, H. Szmacinski, Y. Fu, J. Zhang and K. Nowaczyk, Analyst, 2008, 133, 1308-1346.

577 W. Deng, F. Xie, H. T. M. C. M. Baltar and E. M. Goldys, Phys. Chem. Chem. Phys., 2013, 15, 15695-15708.

578 Q. Cui, F. He, L. Li and H. Mohwald, Adv. Colloid Interface Sci., 2014, 207, 164-177.

579 Y. Jeong, Y. M. Kook, K. Lee and W.-G. Koh, Biosens. Bioelectron., 2018, 111, 102-116.

580 C. Y. Zhang, Q. Y. Han, C. X. Li, M. D. Zhang, L. X. Yan and H. R. Zheng, Appl. Opt., 2016, 55, 9131-9136.

581 Q. Cui, F. He, X. Wang, B. Xia and L. Li, ACS Appl. Mater. Interfaces, 2013, 5, 213-219.

582 R. F. Aroca, G. Y. Teo, H. Mohan, A. R. Guerrero, P. Albella and F. Moreno, J. Phys. Chem. C, 2011, 115, 20419-20424.

583 K. Aslan, M. Wu, J. R. Lakowicz and C. D. Geddes, J. Fluoresc., 2007, 17, 127-131.

584 Y. X. Zhang, L. N. Mandeng, N. Bondre, A. Dragan and C. D. Geddes, Langmuir, 2010, 26, 12371-12376.

585 E. Jang, K. J. Son and W.-G. Koh, Colloid Polym. Sci., 2014, 292, 1355-1364.

586 Z. Mei and L. Tang, Anal. Chem., 2017, 89, 633-639.

587 N. Ma, F. Tang, X. Wang, F. He and L. Li, Macromol. Rapid Commun., 2011, 32, 587-592.

588 J. Asselin, P. Legros, A. Gregoire and D. Boudreau, Plasmonics, 2016, 11, 1369-1376.

589 D. Gontero, A. V. Veglia, A. G. Bracamonte and D. Boudreau, RSC Adv., 2017, 7, 10252-10258.

590 R. Bardhan, N. K. Grady and N. J. Halas, Small, 2008, 4, 1716-1722.

591 Z. Li, S. Chen, J. Li, Q. Liu, Z. Sun, Z. Wang and S. Huang, J. Appl. Phys., 2012, 111, 014310.

592 J. Enderlein, Phys. Chem. Chem. Phys., 2002, 4, 2780-2786. 593 N. Halas, MRS Bull., 2005, 30, 362-367.

594 M. Bauch, K. Toma, M. Toma, Q. Zhang and J. Dostalek, Plasmonics, 2014, 9, 781-799.

595 A. N. Emam, A. S. Mansour, M. B. Mohamed and G. G. Mohamed, in Nanoscience in Medicine, ed. H. K. Daima, P. N. Navya, S. Ranjan, N. Dasgupta and E. Lichtfouse, Springer Nature Switzerland, Cham, 1st edn, 2020, Plasmonic Hybrid Nanocomposites for PlasmonEnhanced Fluorescence and Their Biomedical Applications, vol. 1, pp. 459-488. 
596 A. Sultangaziyev and R. Bukasov, Sens. Bio-Sens. Res., 2020, 30, 100382.

597 J. A. Powell, A. M. Summers, Q. Liu, S. J. Robatjazi, P. Rupp, J. Stierle, C. Trallero-Herrero, M. F. Kling and A. Rudenko, Opt. Express, 2019, 27, 27124-27135.

598 Q. Liu, L. Seiffert, A. Trabattoni, M. C. Castrovilli, M. Galli, P. Rupp, F. Frassetto, L. Poletto, M. Nisoli, E. Rühl, F. Krausz, T. Fennel, S. Zherebtsov, F. Calegari and M. F. Kling, J. Opt., 2018, 20, 024002.
599 P. Rupp, C. Burger, N. G. Kling, M. Kübel, S. Mitra, P. Rosenberger, T. Weatherby, N. Saito, J. Itatani, A. S. Alnaser, M. B. Raschke, E. Rühl, A. Schlander, M. Gallei, L. Seiffert, T. Fennel, B. Bergues and M. F. Kling, Nat. Commun., 2019, 10, 4655.

600 P. Rosenberger, P. Rupp, R. Ali, M. Said Alghabra, S. Sun, S. Mitra, S. A. Khan, R. Dagar, V. Kim, M. Iqbal, J. Schötz, Q. Liu, S. K. Sundaram, J. Kredel, M. Gallei, C. CostaVera, B. Bergues, A. S. Alnaser and M. F. Kling, ACS Photonics, 2020, 7, 1885-1892. 